Acta Crystallographica Section C

Crystal Structure

Communications

ISSN 0108-2701

Tetracarbonyl-1 $\kappa^{2} \boldsymbol{C}, \mathbf{2}_{\kappa^{2}} \boldsymbol{C}$ - - -diphenylphosphido-P:P- ${ }_{\mu}$-iodobis(triphenylphosphine)-1 ${ }_{\kappa} P, 2_{\kappa} P$-diosmium $(O s-O s)$ Benzene Solvate A. W. Herlinger and A. L. Rheingold 


\begin{tabular}{|c|c|c|c|c|c|c|c|c|c|c|c|c|c|c|c|c|c|c|c|c|c|c|c|c|}
\hline 9 & 0 & 0 & 304 & 302 & 5 & 2 & 0 & 1455 & 1463 & -5 & 4 & 0 & 203 & 195 & 9 & 5 & 0 & 377 & 360 & 9 & 7 & 0 & 217 & 211 \\
\hline-6 & 1 & 0 & 607 & 610 & 6 & 2 & 0 & 302 & 285 & -4 & 4 & 0 & 1448 & 1482 & -8 & 6 & 0 & 349 & 332 & 10 & 7 & 0 & 632 & 643 \\
\hline-5 & 1 & 0 & 954 & 958 & 7 & 2 & 0 & 1794 & 1833 & -3 & 4 & 0 & 596 & 604 & -7 & 6 & 0 & 360 & 361 & 11 & 7 & 0 & 468 & 476 \\
\hline-4 & 1 & 0 & 637 & 652 & 9 & 2 & 0 & 930 & 959 & -2 & 4 & 0 & 951 & 978 & -6 & 6 & 0 & 511 & 502 & 12 & 7 & 0 & 476 & 473 \\
\hline-3 & 1 & 0 & 419 & 426 & 10 & 2 & 0 & 727 & 751 & -1 & 4 & 0 & 1646 & 1651 & -5 & 6 & 0 & 785 & 788 & -8 & 8 & 0 & 233 & 222 \\
\hline-2 & 1 & 0 & 326 & 327 & 11 & 2 & 0 & 522 & 522 & 0 & 4 & 0 & 1181 & 1155 & -4 & 6 & 0 & 931 & 930 & -7 & 8 & 0 & 467 & 469 \\
\hline-1 & 1 & 0 & 654 & 611 & 12 & 2 & 0 & 674 & 681 & 2 & 4 & 0 & 755 & 710 & -3 & 6 & 0 & 818 & 817 & -6 & 8 & 0 & 428 & 448 \\
\hline 1 & 1. & 0 & 771 & 771 & -10 & 3 & 0 & 421 & 416 & 3 & 4 & 0 & 194 & 156 & -2 & 6 & 0 & 131 & 116 & -5 & 8 & 0 & 208 & 235 \\
\hline 2 & 1 & 0 & 3247 & 3375 & -9 & 3 & 0 & 233 & 232 & 4 & 4 & 0 & 733 & 731 & -1 & 6 & 0 & 2114 & 2127 & -3 & 8 & 0 & 152 & 66 \\
\hline 3 & 1 & 0 & 288 & 289 & -8 & 3 & 0 & 994 & 975 & 5 & 4 & 0 & 482 & 494 & 0 & 6 & 0 & 442 & 444 & -2 & 8 & 0 & 615 & 626 \\
\hline 4 & 1 & 0 & 1852 & 1841 & -7 & 3 & 0 & 243 & 258 & 6 & 4 & 0 & 708 & 692 & 1 & 6 & 0 & 2621 & 2806 & -1 & 8 & 0 & 386 & 383 \\
\hline 5 & $\perp$ & 0 & 1475 & 1520 & -6 & 3 & 0 & 1743 & 1830 & 7 & 4 & 0 & 901 & 890 & 3 & 6 & 0 & 1330 & 1353 & 0 & 8 & 0 & 576 & 598 \\
\hline 6 & 1 & 0 & 641 & 621 & -5 & 3 & 0 & 730 & 763 & 8 & 4 & 0 & 687 & 717 & 4 & 6 & 0 & 1266 & 1282 & 1 & 8 & 0 & 780 & 785 \\
\hline 7 & 1 & 0 & 1442 & 1473 & -4 & 3 & 0 & 612 & 640 & 9 & 4 & 0 & 897 & 892 & 5 & 6 & 0 & 930 & 925 & 2 & 8 & D & 891 & 916 \\
\hline 9 & 1 & 0 & 780 & 789 & -3 & 3 & 0 & 686 & 685 & 10 & 4 & 0 & 392 & 412 & 6 & 6 & 0 & 1526 & 1570 & 3 & 8 & 0 & 1236 & 1270 \\
\hline 10 & 1 & 0 & 486 & $48 A$ & -2 & 3 & 0 & 691 & 699 & 12 & 4 & 0 & 499 & 486 & 7 & 6 & 0 & 1025 & 1048 & 4 & 8 & 0 & 410 & 373 \\
\hline 1.1 & 1 & 0 & 673 & 660 & -1 & 3 & 0 & 635 & 626 & -10 & 5 & 0 & 433 & 426 & 8 & 6 & 0 & 614 & 613 & 6 & 8 & 0 & 1099 & 1159 \\
\hline 12 & 1 & 0 & 249 & 179 & 0 & 3 & 0 & 1761 & 1796 & -9 & 5 & 0 & 343 & 298 & 10 & 6 & 0 & 441 & 448 & 7 & 8 & 0 & 336 & 317 \\
\hline-11 & 2 & 0 & 395 & 390 & 2 & 3 & 0 & 309 & 318 & -8 & 5 & 0 & 474 & 483 & 11 & 6 & 0 & 275 & 266 & 8 & 8 & 0 & 1441 & 1470 \\
\hline-10 & 2 & 0 & 288 & 270 & 3 & 3 & 0 & 628 & 631 & -7 & 5 & 0 & 260 & 270 & 12 & 6 & 0 & 289 & 298 & 9 & 8 & 0 & 292 & 299 \\
\hline-9 & 2 & 0 & 228 & 213 & 4 & 3 & 0 & 113 & 95 & -6 & 5 & 0 & 1131 & 1138 & -5 & 7 & 0 & 386 & 398 & 10 & 8 & 0 & 709 & 708 \\
\hline-8 & 2 & 0 & 682 & 701 & 5 & 3 & 0 & 1077 & 1076 & -5 & 5 & 0 & 476 & 454 & -4 & 7 & 0 & 553 & 552 & 11 & 8 & 0 & 781 & 789 \\
\hline-7 & 2 & 0 & 542 & 527 & 6 & 3 & 0 & 1135 & 1.167 & -4 & 5 & 0 & 1271 & 1276 & -3 & 7 & 0 & 473 & 508 & 12 & 8 & 0 & 469 & 473 \\
\hline-6 & 2 & 0 & 397 & 405 & 7 & 3 & 0 & 1398 & 1411 & -3 & 5 & 0 & 1316 & 1299 & -2 & 7 & 0 & 167 & 144 & -7 & 9 & 0 & 804 & 782 \\
\hline-5 & 2 & 0 & 402 & 365 & 8 & 3 & 0 & 570 & 576 & -2 & 5 & 0 & 464 & 464 & -1 & 7 & 0 & 916 & 907 & -5 & 9 & 0 & 789 & 771 \\
\hline-4 & 2 & 0 & 19 & 31 & 9 & 3 & 0 & 973 & 975. & -1 & 5 & 0 & 2748 & 2745 & 0 & 7 & 0 & 877 & 899 & -4 & 9 & 0 & 190 & 204 \\
\hline
\end{tabular}

Observed and calculated structure factors: $\quad\left[\mathrm{OS}(\mathrm{CO}) \mathrm{PPH}_{3}\right]_{2}$ (MU-I) (MU-PPH ${ }_{2}$ )

Page 2

h \& 1 10Fo 10FC

$\begin{array}{lllll}-3 & 9 & 0 & 352 & 343\end{array}$ $\begin{array}{llllll}-2 & 9 & 0 & 517 & 504\end{array}$ $\begin{array}{lllll}-1 & 9 & 0 & 692 & 708\end{array}$ $0 \quad 900202 \quad 215$ 190678660 $290 \quad 359 \quad 360$ $4 \quad 9 \quad 0 \quad 283 \quad 268$ $690 \quad 375 \quad 370$ $\begin{array}{lllll}7 & 9 & 0 & 767 & 750\end{array}$ $890 \quad 10321015$ $\begin{array}{lllll}9 & 9 & 0 & 376 & 390\end{array}$ $\begin{array}{lllll}10 & 9 & 0 & 421 & 419\end{array}$ $\begin{array}{lllll}11 & 9 & 0 & 720 & 745\end{array}$ $\begin{array}{lllll}-5 & 10 & 0 & 1131 & 1117\end{array}$ $\begin{array}{lllll}-3 & 10 & 0 & 630 & 640\end{array}$ $\begin{array}{lllll}-2 & 1.0 & 0 & 541 & 546\end{array}$ $\begin{array}{lllll}-1 & 10 & 0 & 240 & 228\end{array}$ 0100 854 845 $110 \quad 0 \quad 600 \quad 590$ $\begin{array}{lllll}2 & 10 & 0 & 281 & 288\end{array}$ $\begin{array}{lllll}4 & 10 & 0 & 406 & 384\end{array}$ $\begin{array}{lllll}6 & 10 & 0 & 244 & 247\end{array}$ $\begin{array}{lllll}7 & 10 & 0 & 587 & 594\end{array}$ $\begin{array}{lllll}8 & 10 & 0 & 506 & 504\end{array}$ $\begin{array}{lllll}9 & 10 & 0 & 219 & 198\end{array}$ $\begin{array}{lllll}10 & 10 & 0 & 328 & 299\end{array}$ $\begin{array}{lllll}11 & 10 & 0 & 312 & 320\end{array}$ $\begin{array}{lllll}-5 & 11 & 0 & 757 & 749\end{array}$ $\begin{array}{lllll}-4 & 11 & 0 & 298 & 289\end{array}$ $\begin{array}{lllll}-3 & 11 & 0 & 628 & 629\end{array}$ $\begin{array}{lllll}-2 & 11 & 0 & 557 & 546\end{array}$ $\begin{array}{lllll}-1 & 11 & 0 & 197 & 231\end{array}$ $\begin{array}{lllll}0 & 11 & 0 & 1186 & 1175\end{array}$ $\begin{array}{lllll}2 & 11 & 0 & 893 & 920\end{array}$ $h \mathrm{k} 1$ 10FO 10FC

$\begin{array}{lllll}411 & 0 & 619 & 623\end{array}$

$\begin{array}{lllll}5 & 11 & 0 & 593 & 571\end{array}$

$\begin{array}{lllll}6 & 11 & 0 & 584 & 593\end{array}$

$\begin{array}{lllll}7 & 11 & 0 & 205 & 174\end{array}$

$\begin{array}{lllll}10 & 11 & 0 & 275 & 281\end{array}$

$\begin{array}{lllll}-4 & 12 & 0 & 372 & 343\end{array}$

$\begin{array}{lllll}-3 & 12 & 0 & 262 & 318\end{array}$

$\begin{array}{lllll}-2 & 12 & 0 & 314 & 315\end{array}$

$\begin{array}{lllll}0 & 12 & 0 & 876 & 868\end{array}$

$\begin{array}{lllll}1 & 12 & 0 & 513 & 522\end{array}$

$\begin{array}{lllll}2 & 12 & 0 & 1342 & 1428\end{array}$

$\begin{array}{lllll}3 & 12 & 0 & 389 & 372\end{array}$

$4 \begin{array}{llll}12 & 0 & 639 & 632\end{array}$

$\begin{array}{lllll}5 & 12 & 0 & 1152 & 1159\end{array}$

$\begin{array}{lllll}6 & 12 & 0 & 555 & 548\end{array}$

$\begin{array}{lllll}7 & 12 & 0 & 817 & 821\end{array}$

$\begin{array}{lllll}8 & 12 & 0 & 316 & 288\end{array}$

$\begin{array}{lllll}9 & 12 & 0 & 251 & 226\end{array}$

$\begin{array}{lllll}11 & 12 & 0 & 274 & 263\end{array}$

$\begin{array}{lllll}-1 & 13 & 0 & 198 & 190\end{array}$

$\begin{array}{lllll}0 & 13 & 0 & 433 & 461\end{array}$

$\begin{array}{lllll}1 & 13 & 0 & 651 & 615\end{array}$

$\begin{array}{lllll}2 & 13 & 0 & 899 & 949\end{array}$

$\begin{array}{lllll}3 & 13 & 0 & 475 & 470\end{array}$

$\begin{array}{lllll}4 & 13 & 0 & 484 & 480\end{array}$

$513 \bigcirc 800 \quad 804$

$\begin{array}{llll}13 & 0 & 282 & 298\end{array}$

$\begin{array}{lllll}7 & 13 & 0 & 1088 & 1079\end{array}$

$\begin{array}{lllll}9 & 13 & 0 & 674 & 677\end{array}$

$\begin{array}{lllll}1 & 14 & 0 & 405 & 398\end{array}$

$214 \quad 0 \quad 533 \quad 545$

$\begin{array}{lllll}314 & 0 & 394 & 407\end{array}$

$\begin{array}{lllll}14 & 0 & 427 & 417\end{array}$

$514 \bigcirc 350 \quad 343$ h k 1 10Fo 10FC

$\begin{array}{lllll}7 & 14 & 0 & 679 & 678\end{array}$

$\begin{array}{lllll}9 & 14 & 0 & 562 & 583\end{array}$

$\begin{array}{lllll}0 & 15 & 0 & 330 & 314\end{array}$

4150 o 301280

$\begin{array}{lllll}7 & 15 & 0 & 177 & 167\end{array}$

$\begin{array}{lllll}8 & 15 & 0 & 297 & 273\end{array}$

$\begin{array}{lllll}5 & 16 & 0 & 210 & 198\end{array}$

$\begin{array}{llll}-4-15 & 1 & 211 & 229\end{array}$

$\begin{array}{llll}-1-15 & 1 & 414 & 370\end{array}$

$\begin{array}{llll}0-15 & 1 & 402 & 334\end{array}$

$\begin{array}{llll}-9-14 & 1 & 241 & 182\end{array}$

$-8-141523 \quad 514$

$-6-141235 \quad 240$

$-3-14 \quad 1 \quad 466 \quad 402$

$\begin{array}{llll}1-14 & 1 & 411 & 396\end{array}$

$2-14 \quad 1 \quad 245 \quad 213$

$-10-13 \quad 1 \quad 608 \quad 599$

$\begin{array}{llll}-8-13 & 1 & 917 & 914\end{array}$

$\begin{array}{llll}-7-13 & 1 & 276 & 279\end{array}$

$\begin{array}{llll}-6-13 & 1 & 866 & 857\end{array}$

$\begin{array}{llll}-4-13 & 1 & 376 & 374\end{array}$

$\begin{array}{llll}-3-13 & 1 & 541 & 530\end{array}$

$\begin{array}{llll}-1-13 & 1 & 672 & 660\end{array}$

0-13 $1440 \quad 454$

$-10-12 \quad 1 \quad 463 \quad 454$

$\begin{array}{llll}-8-12 & 1 & 722 & 717\end{array}$

$\begin{array}{llll}-7-12 & 1 & 676 & 675\end{array}$

$\begin{array}{llll}-6-12 & 1 & 1117 & 1122\end{array}$

$\begin{array}{llll}-5-12 & 1 & 706 & 686\end{array}$

$-4-12 \quad 1 \quad 852 \quad 854$

$-3-12 \quad 1 \quad 833 \quad 816$

$\begin{array}{llll}-1-12 & 1 & 1049 & 1036\end{array}$

$\begin{array}{llll}-12 & 1 & 522 & 520\end{array}$

$\begin{array}{llll}4-12 & 1 & 350 & 326\end{array}$ h $k$ I 10FO 10FC

$\begin{array}{llll}-10-11 & 1 & 294 & 270\end{array}$ $\begin{array}{llll}-7-11 & 1 & 693 & 687\end{array}$ $\begin{array}{llll}-6-11 & 1 & 673 & 678\end{array}$ $\begin{array}{llll}-5-11 & 1 & 1177 & 1159\end{array}$ $\begin{array}{llll}-4-11 & 1 & 943 & 911\end{array}$ $\begin{array}{llll}-3-11 & 1 & 772 & 748\end{array}$

$\begin{array}{llll}-1-11 & 1 & 1188 & 1201\end{array}$

$0-11 \quad 1 \quad 484 \quad 469$

1-11 110021021

$\begin{array}{llll}2-11 & 1 & 316 & 326\end{array}$

$3-11 \quad 1 \quad 264 \quad 235$

$4-11 \quad 1 \quad 588 \quad 610$

$\begin{array}{llll}5-11 & 1 & 239 & 192\end{array}$

$\begin{array}{llll}-9-10 & 1 & 353 & 362\end{array}$

$-6-10 \quad I \quad 232 \quad 275$

$\begin{array}{llll}-5-10 & 1 & 927 & 955\end{array}$

$-3-10 \quad 1 \quad 749 \quad 740$

$-2-10 \quad 1 \quad 173 \quad 141$

$-1-10 \quad 1 \quad 564 \quad 555$

$\begin{array}{llll}0-10 & 1 & 711 & 721\end{array}$

1-10 110501081

$2-10 \quad 1 \quad 634 \quad 646$

$\begin{array}{cccc}3-10 & 1 & 309 & 312\end{array}$

$4-10 \quad 1 \quad 918 \quad 920$

$\begin{array}{llll}6-10 & 1 & 927 & 932\end{array}$

$\begin{array}{lllll}-12 & -9 & 1 & 755 & 759\end{array}$

$\begin{array}{lllll}-10 & -9 & 1 & 513 & 515\end{array}$

$\begin{array}{lllll}-9 & -9 & 1 & 279 & 335\end{array}$

$\begin{array}{lllll}-7 & -9 & 1 & 626 & 627\end{array}$

$\begin{array}{llll}-9 & 1 & 529 & 530\end{array}$

$\begin{array}{lllll}-5 & -9 & 1 & 217 & 170\end{array}$

$\begin{array}{lllll}-3 & -9 & 1 & 507 & 500\end{array}$

$\begin{array}{llll}-9 & 1 & 168 & 156\end{array}$

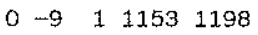

$\mathrm{h} \mathrm{k}$ l 10FO 10FC

$\begin{array}{lllll}1 & -9 & 1 & 768 & 783\end{array}$

$\begin{array}{lllll}2 & -9 & 1 & 838 & 828\end{array}$

$\begin{array}{lllll}3 & -9 & 1 & 264 & 255\end{array}$

$\begin{array}{lllll}4 & -9 & 1 & 680 & 685\end{array}$

$\begin{array}{lllll}5 & -9 & 1 & 289 & 236\end{array}$

$\begin{array}{lllll}6 & -9 & 1 & 973 & 958\end{array}$

$\begin{array}{lllll}-12 & -8 & 1 & 775 & 794\end{array}$

$\begin{array}{lllll}-11 & -8 & 1 & 547 & 548\end{array}$

$\begin{array}{lllll}-10 & -8 & 1 & 815 & 847\end{array}$

$\begin{array}{llllll}-9 & -8 & 1 & 723 & 731\end{array}$

$\begin{array}{lllll}-7 & -8 & 1 & 1051 & 1034\end{array}$

$\begin{array}{lllll}-5 & -8 & 1 & 737 & 733\end{array}$

$\begin{array}{lllll}-4 & -8 & 1 & 410 \quad 406\end{array}$

$\begin{array}{lllll}-2 & -8 & 1 & 451 & 463\end{array}$

$\begin{array}{lllll}-1 & -8 & 1 & 838 & 852\end{array}$

$\begin{array}{lllll}2 & -8 & 1 & 799 & 835\end{array}$

$\begin{array}{lllll}3 & -8 & 1 & 544 & 543\end{array}$

$\begin{array}{lllll}4 & -8 & 1 & 609 & 597\end{array}$

$\begin{array}{lllll}5 & -8 & 1 & 400 & 388\end{array}$

$\begin{array}{lllll}6 & -8 & 1 & 464 & 468\end{array}$

$\begin{array}{lllll}7 & -8 & 1 & 437 & 466\end{array}$

$\begin{array}{lllll}8 & -8 & 1 & 525 & 518\end{array}$

$\begin{array}{lllll}-12 & -7 & 1 & 371 & 412\end{array}$

$\begin{array}{lllll}-11 & -7 & 1 & 754 & 763\end{array}$

$\begin{array}{rrrrr}-10 & -7 & 1 & 620 & 638 \\ -9 & -7 & 1 & 790 & 790\end{array}$

$\begin{array}{lllll}-7 & -7 & 1 & 987 & 965\end{array}$

$\begin{array}{lllll}-6 & -7 & 1 & 786 & 802\end{array}$

$\begin{array}{llllll}-5 & -7 & 1 & 1471 & 1493\end{array}$

$\begin{array}{lllll}-4 & -7 & 1 & 722 & 726\end{array}$

$\begin{array}{lllll}-3 & -7 & 1 & 187 & 205\end{array}$

$\begin{array}{lllll}-2 & -7 & 1 & 1713 & 1669\end{array}$

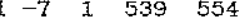

$\begin{array}{lllll}0 & -7 & 1 & 1281 & 1242\end{array}$ 


\begin{tabular}{|c|c|c|c|c|c|c|c|c|c|c|c|c|c|c|c|c|c|c|c|c|c|c|c|c|}
\hline-6 & -6 & 1 & 1440 & 1446 & -5 & -4 & 1 & 149 & 132 & 8 & -3 & 1 & 411 & 401 & -2 & -1 & 1 & 372 & 388 & 2 & 1 & 1 & 992 & 968 \\
\hline-5 & -6 & 1 & 1658 & 1673 & -4 & -4 & 1 & 1642 & 1620 & 9 & -3 & 1 & 838 & 823 & -1 & -1 & 1 & 2138 & 2133 & 3 & 1 & 1 & 2108 & 2090 \\
\hline-4 & -6 & 1 & 1154 & 1182 & -3 & -4 & 1 & 298 & 299 & 10 & -3 & 1 & 461 & 462 & 0 & -1 & 1 & 449 & 449 & 4 & 1 & 1. & 652 & 661 \\
\hline-2 & -6 & 1 & 2522 & 2504 & -2 & -4 & 1 & 1701 & 1645 & -12 & -2 & 1. & 655 & 665 & 1 & -1 & 1 & 1175 & 1142 & 5 & 1 & 1 & 228 & 190 \\
\hline-1 & -6 & 1 & 182 & 129 & -1 & -4 & 1 & 718 & 740 & -11 & -2 & 1 & 817 & 813 & 2 & -1 & 1 & 507 & 484 & 6 & 1 & 1 & 2019 & 2029 \\
\hline 0 & -6 & 1 & 2517 & 2496 & 0 & -4 & 1 & 2261 & 2193 & -10 & -2 & 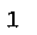 & 565 & 586 & 3 & -1 & 1 & 221 & 242 & 7 & 1. & 1 & 193 & 196 \\
\hline 1 & -6 & 1 & 373 & 359 & 1 & -4 & 1 & 1505 & 1489 & -9 & -2 & 1 & 495 & 494 & 4 & -1 & 1 & 1105 & 1125 & 8 & . & 1 & 1097 & 1102 \\
\hline 2 & -6 & 1 & 875 & 914 & 2 & -4 & 1 & 1513 & 1497 & -8 & -2 & 1 & 1281 & 1294 & 5 & -1 & 1 & 344 & 343 & 10 & & 1 & 373 & 371 \\
\hline 3 & -6 & 1 & 297 & 266 & 3 & -4 & 1 & 1523 & 1497 & -6 & -2 & 1 & 1938 & 1960 & 8 & -1 & 1 & 791 & 810 & -9 & 2 & 1 & 226 & 200 \\
\hline 4 & -6 & 1 & 258 & 279 & 4 & -4 & 1 & 224 & 198 & -5 & -2 & 1 & 1017 & 961 & 9 & -1 & 1 & 338 & 328 & -7 & & 1 & 367 & 380 \\
\hline 5 & -6 & 1 & 779 & 795 & 5 & -4 & 1 & 1371 & 1395 & -4 & -2 & 1 & 494 & 508 & 10 & -1 & 1 & 245 & 285 & $-G$ & 2 & 1. & 219 & 219 \\
\hline 6 & -6 & 1 & 585 & 611 & 6 & -4 & 1 & 234 & 236 & -3 & -2 & 1 & 504 & 472 & -10 & 0 & 1 & 710 & 723 & -5 & 2 & 1 & 890 & 889 \\
\hline 11 & -5 & 1 & 294 & 296 & 7 & -4 & 1 & 1365 & 1356 & -2 & -2 & 1 & 627 & 641 & -8 & 0 & 1 & 689 & 737 & -4 & 2 & 1 & 317 & 305 \\
\hline-10 & -5 & 1 & 190 & 75 & 9 & -4 & 1 & 423 & 431 & -1 & -2 & 1 & 867 & 793 & -7 & 0 & 1 & 750 & 753 & -3 & 2 & 1 & 1.225 & 1199 \\
\hline-9 & -5 & 1 & 349 & 341 & 10 & -4 & 1 & 440 & 469 & 0 & -2 & 1 & 699 & 649 & -6 & 0 & 1 & 881 & 892 & -2 & 2 & 1 & 478 & 442 \\
\hline-8 & -5 & 1 & 305 & 325 & -12 & -3 & 1 & 262 & 258 & 1 & -2 & 1 & 164 & 102 & -5 & 0 & 1 & 1387 & 1424 & -1 & 2. & 1 & 553 & 514 \\
\hline-6 & -5 & 1 & 1707 & 1716 & -11 & -3 & 1 & 720 & 720 & 3 & -2 & 1 & 1026 & 1059 & -4 & 0 & 1 & 1280 & 1258 & 0 & 2 & 1 & 1170 & 1075 \\
\hline-5 & -5 & 1 & 997 & 982 & -10 & -3 & 1 & 281 & 263 & 4 & -2 & 1 & 229 & 191 & -3 & 0 & 1 & 1567 & 1513 & 1 & 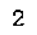 & 1 & 1685 & 1657 \\
\hline-4 & -5 & 1 & 1631 & 1655 & -8 & -3 & 1 & 1129 & 1148 & 5 & -2 & 1 & 235 & 230 & -2 & 0 & 1 & 2009 & 1949 & 2 & 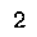 & 1 & 2046 & 2044 \\
\hline-3 & -5 & 1. & 1055 & 1040 & -7 & -3 & 1 & 520 & 502 & 6 & -2 & 1 & 994 & 1004 & -1 & 0 & 1 & 3527 & 3588 & 3 & 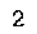 & 1 & 1622 & 1625 \\
\hline-2 & -5 & 1 & 1675 & 1605 & -6 & -3 & 1 & 1501 & 1476 & 7 & -2 & 1 & 1106 & 1118 & 1 & 0 & 1 & 3637 & 3603 & 4 & 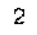 & 1 & $5 \Delta 4$ & 519 \\
\hline-1 & -5 & 1 & 1329 & 1332 & -5 & -3 & 1 & 1093 & 1105 & 8 & -2 & 1 & 865 & 865 & 2 & 0 & 1 & 107 & 77 & 5 & 2 & 1 & 273 & 464 \\
\hline 0 & -5 & 1 & 2849 & 2777 & -4 & -3 & 1 & 174 & 157 & 9 & -2 & 1 & 800 & 785 & 3 & 0 & 1 & 247 & 241. & 6 & $?$ & 1 & 1850 & 1872 \\
\hline 1 & -5 & 1 & 422 & 419 & -3 & -3 & $I$ & 1224 & 1174 & 10 & -2 & 1 & 365 & 359 & 4 & 0 & 1 & 1274 & 1274 & 7 & 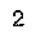 & 1 & 385 & 397 \\
\hline 2 & -5 & 1 & 1803 & 1846 & -2 & -3 & 1 & 781 & 737 & -12 & -1 & 1 & 666 & 647 & 5 & 0 & 1 & 179 & 127 & 8 & f & 1 & 1444 & 1.467 \\
\hline 3 & -5 & 1 & 360 & 368 & -1 & -3 & 1 & 1082 & 1050 & -11 & -1 & 1 & 482 & 474 & 6 & 0 & 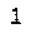 & 1367 & 1434 & 9 & 2 & 1 & 424 & 456 \\
\hline
\end{tabular}

Observed and calculated structure factors: $\quad\left[\mathrm{OS}(\mathrm{CO}) \mathrm{PPH}_{3} \mathrm{l}_{2}\right.$ (MU-I) (MJ-PPH$)$

Page 4

h $\mathrm{k} \quad 1$ 10FO 10FC

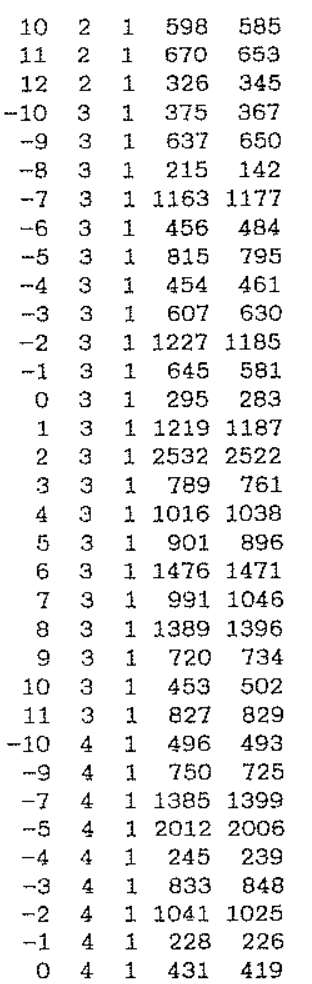

h $\mathrm{k} 1 \mathrm{IOFO} 10 \mathrm{FC}$

141972956

$\begin{array}{lllll}2 & 4 & 1 & 161 & 153\end{array}$

$\begin{array}{lllll}3 & 4 & 1 & 231 & 233\end{array}$

$441518 \quad 529$

$541224 \quad 225$

641603612

$7 \quad 4 \quad 1 \quad 12181229$

$\begin{array}{lllll}8 & 4 & 1 & 1171 & 1152\end{array}$

$941823 \quad 839$

$10 \triangle 1486495$

$1141676 \quad 666$

$\begin{array}{lllll}-10 & 5 & 1 & 272 & 290\end{array}$

$\begin{array}{lllll}-9 & 5 & 1 & 602 & 579\end{array}$

$\begin{array}{lllll}-7 & 5 & 1 & 802 & 811\end{array}$

$\begin{array}{lllll}-6 & 5 & 1 & 499 & 505\end{array}$

$\begin{array}{lllll}-5 & 5 & 1 & 1721 & 1713\end{array}$

$\begin{array}{lllll}-4 & 5 & 1 & 624 & 612\end{array}$

$\begin{array}{lllll}-3 & 5 & 1 & 1466 & 1478\end{array}$

$\begin{array}{lllll}-2 & 5 & 1 & 909 & 897\end{array}$

$\begin{array}{lllll}-1 & 5 & 1 & 359 & 349\end{array}$

- 5115031480

$\begin{array}{lllll}1 & 5 & 1 & 1351 & 1335\end{array}$

$\begin{array}{lllll}2 & 5 & 1 & 1152 & 1143\end{array}$

$\begin{array}{lllll}4 & 5 & 1 & 289 & 265\end{array}$

$\begin{array}{lllll}5 & 5 & 1 & 513 & 524\end{array}$

$\begin{array}{lllll}6 & 5 & 1 & 471 & 476\end{array}$

$\begin{array}{lllll}7 & 5 & 1 & 445 & 434\end{array}$

$851350 \quad 358$

$\begin{array}{lllll}9 & 5 & 1 & 674 & 687\end{array}$

$\begin{array}{lllll}10 & 5 & 1 & 496 & 481\end{array}$

$\begin{array}{llllll}-9 & 6 & 1 & 505 & 510\end{array}$

$\begin{array}{lllll}-7 & 6 & 1 & 435 & 442\end{array}$

$\begin{array}{lllll}-6 & 6 & 1 & 534 & 568\end{array}$

$\begin{array}{lllll}-5 & 6 & 1 & 582 & 585\end{array}$ h $k$ I 1OFO 1OFC

$\begin{array}{lllll}-4 & 6 & 1 & 1105 & 1126\end{array}$

$\begin{array}{lllll}-3 & 6 & 1 & 790 & 765\end{array}$

$\begin{array}{lllll}-2 & 6 & 1 & 1176 & 1187\end{array}$

$\begin{array}{lllll}-1 & 6 & 1 & 1107 & 1082\end{array}$

$\begin{array}{lllll}0 & 6 & 1 & 2119 & 2149\end{array}$

$\begin{array}{lllll}1 & 6 & 1 & 546 & 527\end{array}$

$26511894 \quad 1915$

$\begin{array}{lllll}3 & 6 & 1 & 569 & 581\end{array}$

$461358 \quad 370$

$\begin{array}{lllll}5 & 6 & 1 & 1484 & 1477\end{array}$

$\begin{array}{lllll}6 & 6 & 1 & 457 & 471\end{array}$

$\begin{array}{lllll}7 & 6 & 1 & 974 & 1000\end{array}$

$\begin{array}{lllll}8 & 6 & 1 & 633 & 642\end{array}$

$\begin{array}{lllll}-8 & 7 & 1 & 202 & 172\end{array}$

$\begin{array}{lllll}-6 & 7 & 1 & 372 & 339\end{array}$

$\begin{array}{lllll}-4 & 7 & 1 & 1092 & 1093\end{array}$

$\begin{array}{lllll}-3 & 7 & 1 & 390 & 379\end{array}$

$\begin{array}{lllll}-2 & 7 & 1 & 1013 & 1018\end{array}$

$\begin{array}{lllll}-1 & 7 & 1 & 593 & 570\end{array}$

$\begin{array}{lllll}0 & 7 & 1 & 1951 & 1930\end{array}$

17116421656

$\begin{array}{lllll}2 & 7 & 1 & 2921 & 2956\end{array}$

$\begin{array}{lllll}3 & 7 & 1 & 385 & 423\end{array}$

$\begin{array}{lllll}4 & 7 & 1 & 831 & 873\end{array}$

$\begin{array}{llllll}5 & 7 & 1 & 1170 & 1177\end{array}$

$\begin{array}{lllll}7 & 7 & 1 & 1855 & 1847\end{array}$

$\begin{array}{lllll}9 & 7 & 1 & 822 & 814\end{array}$

$\begin{array}{lllll}10 & 7 & 1 & 223 & 247\end{array}$

$\begin{array}{lllll}11 & 7 & 1 & 307 & 326\end{array}$

$\begin{array}{lllll}-6 & 8 & 1 & 284 & 282\end{array}$

$\begin{array}{lllll}-5 & 8 & 1 & 271 & 260\end{array}$

$\begin{array}{lllll}-4 & 8 & 1 & 261 & 283\end{array}$

$\begin{array}{lllll}-3 & 8 & 1 & 272 & 278\end{array}$

$\begin{array}{lllll}-2 & 8 & 1 & 523 & 541\end{array}$ h $k$ IOFO 1OFC

$\begin{array}{lllll}-1 & 8 & 1 & 327 & 299\end{array}$

$\begin{array}{lllll}0 & 8 & 1 & 687 & 712\end{array}$

$\begin{array}{lllll}1 & 8 & 1 & 1175 & 1175\end{array}$

$\begin{array}{lllll}2 & 8 & 1 & 1673 & 1620\end{array}$

$\begin{array}{lllll}3 & 8 & 1 & 1061 & 1075\end{array}$

$\begin{array}{lllll}4 & 8 & 1 & 781 & 788\end{array}$

$\begin{array}{lllll}5 & 8 & 1 & 977 & 985\end{array}$

$\begin{array}{lllll}6 & 8 & 1 & 297 & 311\end{array}$

$\begin{array}{lllll}7 & 8 & 1 & 1363 & 1380\end{array}$

$\begin{array}{lllll}8 & 8 & 1 & 528 & 541\end{array}$

$\begin{array}{lllll}9 & 8 & 1 & 1159 & 1177\end{array}$

$\begin{array}{lllll}10 & 8 & 1 & 343 & 332\end{array}$

$\begin{array}{lllll}11 & 8 & 1 & 508 & 475\end{array}$

$\begin{array}{lllll}12 & 8 & 1 & 514 & 497\end{array}$

$\begin{array}{lllll}-6 & 9 & 1 & 667 & 674\end{array}$

$\begin{array}{lllll}-4 & 9 & 1 & 284 & 327\end{array}$

$\begin{array}{lllll}-1 & 9 & 1 & 539 & 524\end{array}$

$\begin{array}{lllll}0 & 9 & 1 & 430 & 433\end{array}$

$\begin{array}{lllll}1 & 9 & 1 & 233 & 204\end{array}$

$\begin{array}{lllll}2 & 9 & 1 & 341 & 299\end{array}$

$\begin{array}{lllll}3 & 9 & 1 & 1048 & 1059\end{array}$

491535540

$\begin{array}{lllll}5 & 9 & 1 & 187 & 234\end{array}$

$\begin{array}{lllll}6 & 9 & 1 & 429 & 426\end{array}$

$\begin{array}{lllll}7 & 9 & 1 & 671 & 664\end{array}$

$\begin{array}{lllll}8 & 9 & 1 & 1007 & 978\end{array}$

$\begin{array}{lllll}9 & 9 & 1 & 910 & 917\end{array}$

$\begin{array}{rrrrr}10 & 9 & 1 & 673 & 693\end{array}$

$\begin{array}{lllll}11 & 9 & 1 & 440 & 417\end{array}$

$\begin{array}{lllll}12 . & 9 & 1 & 704 & 712\end{array}$

$\begin{array}{lllll}-6 & 10 & 1 & 834 & 800\end{array}$

$\begin{array}{lllll}-5 & 10 & 1 & 225 & 231\end{array}$

$\begin{array}{lllll}-4 & 10 & 1 & 918 & 949\end{array}$

$\begin{array}{lllll}-1 & 10 & 1 & 589 & 598\end{array}$ $\mathrm{h} \mathrm{k} 1$ 10FO 1OFC

$\begin{array}{lllll}0 & 10 & 1 & 331 & 367\end{array}$ $\begin{array}{lllll}1 & 10 & 1 & 806 & 778\end{array}$ $\begin{array}{lllll}2 & 10 & 1 & 503 & 507\end{array}$ $\begin{array}{lllll}6 & 10 & 1 & 272 & 269\end{array}$ $\begin{array}{lllll}7 & 10 & 1 & 185 & 120\end{array}$ $\begin{array}{lllll}8 & 10 & 1 & 757 & 786\end{array}$ $\begin{array}{lllll}9 & 10 & 1 & 348 & 334\end{array}$ $\begin{array}{lllll}10 & 10 & 1 & 621 & 618\end{array}$ $\begin{array}{lllll}12 & 10 & 1 & 645 & 617\end{array}$ $\begin{array}{lllll}-5 & 11 & 1 & 464 & 455\end{array}$ $\begin{array}{lllll}-4 & 11 & 1 & 969 & 983\end{array}$ $\begin{array}{lllll}-3 & 11 & 1 & 223 & 258\end{array}$ $\begin{array}{lllll}-2 & 11 & 1 & 485 & 478\end{array}$ $\begin{array}{lllll}-1 & 11 & 1 & 704 & 713\end{array}$ $\begin{array}{lllll}1 & 11 & 1 & 921 & 918\end{array}$ $\begin{array}{lllll}3 & 11 & 1 & 380 & 391\end{array}$ $\begin{array}{lllll}4 & 11 & 1 & 189 & 139\end{array}$ $\begin{array}{lllll}5 & 11 & 1 & 211 & 233\end{array}$ $\begin{array}{lllll}6 & 11 & 1 & 392 & 356\end{array}$ $811 \quad 1 \quad 315 \quad 320$ $\begin{array}{lllll}10 & 11 & 1 & 330 & 306\end{array}$ $\begin{array}{lllll}-4 & 12 & 1 & 442 & 428\end{array}$ $\begin{array}{lllll}-3 & 12 & 1 & 507 & 484\end{array}$ $\begin{array}{lllll}-2 & 12 & 1 & 272 & 292\end{array}$

$\begin{array}{lllll}-1 & 12 & 1 & 629 & 613\end{array}$ $\begin{array}{lllll}0 & 12 & 1 & 255 & 246\end{array}$ $\begin{array}{lllll}1 & 12 & 1 & 1064 & 1060\end{array}$ $\begin{array}{lllll}2 & 12 & 1 & 342 & 358\end{array}$ $\begin{array}{lllll}3 & 12 & 1 & 998 & 1030\end{array}$

$\begin{array}{lllll}4 & 12 & 1 & 489 & 533\end{array}$

$\begin{array}{lllll}5 & 12 & 1 & 360 & 373\end{array}$

$\begin{array}{lllll}6 & 12 & 1 & 833 & 847\end{array}$

$\begin{array}{lllll}8 & 12 & 1 & 268 & 320\end{array}$ $\begin{array}{lllll}-3 & 13 & 1 & 400 & 415\end{array}$ 


\begin{tabular}{|c|c|c|c|c|c|c|c|c|c|c|c|c|c|c|c|c|c|c|c|c|c|c|}
\hline 813 & 1 & 831 & 829 & $-8-12$ & 2 & 408 & 426 & & -10 & 2 & 801 & 792 & -10 & -7 & 2 & 896 & 897 & -7 & -5 & 2 & 38 & 1196 \\
\hline 1013 & 1 & 380 & 358 & $-7-12$ & 2 & 813 & 818 & -11 & -9 & 2 & 450 & 476 & -8 & -7 & 2 & 967 & 973 & -6 & -5 & 2 & 671 & 695 \\
\hline 014 & 1 & 221 & 237 & $-6-12$ & 2 & 947 & 978 & -8 & -9 & 2 & 405 & 380 & -7 & -7 & 2 & 458 & 479 & -5 & -5 & 2 & 2205 & 2222 \\
\hline 114 & 1 & 353 & 371 & $-5-12$ & 2 & 1038 & 1025 & -7 & -9 & 2 & 356 & 350 & -6 & -7 & 2 & 1210 & 1210 & -4 & -5 & 2 & 1279 & 1237 \\
\hline 214 & 1 & 646 & 615 & $-4-12$ & 2 & 589 & 550 & -5 & -9 & 2 & 387 & 382 & -5 & -7 & 2 & 669 & 681 & -3 & -5 & 2 & 1846 & 185.1 \\
\hline 314 & 1 & 586 & 591 & $-3-12$ & 2 & 203 & 265 & -4 & -9 & 2 & 1009 & 999 & -4 & -7 & 2 & 962 & 963 & -1 & -5 & 2 & 2096 & 2186 \\
\hline 414 & 1 & 551 & 527 & $-2-12$ & 2 & 685 & 680 & -2 & -9 & 2 & 721 & 763 & -3 & -7 & 2 & 631 & 624 & 0 & -5 & 2 & 1381 & 1329 \\
\hline $5 \quad 14$ & 1 & 209 & 194 & $0-12$ & 2 & 754 & 760 & -1 & -9 & 2 & 422 & 414 & -1 & -7 & 2 & 1041 & 1095 & 1 & -5 & 2 & 2245 & 2209 \\
\hline 614 & 1 & 573 & 577 & $1-12$ & 2 & 248 & 218 & 0 & -9 & 2 & 268 & 213 & 0 & -7 & 2 & 562 & 559 & 2 & -5 & 2 & 425 & 428 \\
\hline 714 & 1 & 275 & 282 & $-11-11$ & 2 & 403 & 391 & 1 & -9 & 2 & 1198 & 1189 & $I$ & -7 & 2 & 178 & 149 & 3 & -5 & 2 & 938 & 955 \\
\hline 814 & 1 & 890 & 836 & $-9-11$ & 2 & 481 & 490 & 2 & -9 & 2 & 475 & 464 & 3 & -7 & 2 & 702 & 727 & 4 & -5 & 2 & 601 & 6.30 \\
\hline 015 & 1 & 246 & 166 & $-8-11$ & 2 & 421 & 402 & 3 & -9 & 2 & 890 & 910 & 4 & -7 & 2 & 267 & 299 & 5 & -5 & 2 & 179 & 249 \\
\hline 215 & 1 & 208 & 207 & $-7-11$ & 2 & 634 & 633 & 5 & -9 & 2 & 867 & 881 & 5 & -7 & 2 & 289 & 304 & 6 & -5 & 2 & 1017 & 1049 \\
\hline 315 & 1 & 220 & 238 & $-6-11$ & 2 & 1287 & 1289 & 6 & -9 & 2 & 271 & 347 & 6 & -7 & 2 & 319 & 297 & 7 & -5 & 2 & 310 & 343 \\
\hline 415 & 1 & 340 & 352 & $-5-11$ & 2 & 1076 & 1078 & 7 & -9 & 2 & 878 & 868 & 8 & -7 & 2 & 601 & 615 & 8 & -5 & 2 & 371 & $3 \leq 2$ \\
\hline 515 & 1 & 262 & 259 & $-4-11$ & 2 & 974 & 953 & -12 & -8 & 2 & 573 & 593 & -12 & -6 & 2 & 571 & 557 & -12 & -4 & 2 & 286 & 252 \\
\hline 715 & 1 & 281 & 328 & $-3-11$ & 2 & 599 & 592 & -11 & -8 & 2 & 840 & 852 & -10 & -6 & 2 & 1054 & 1087 & -8 & -4 & 2 & 289 & 285 \\
\hline 815 & 1 & 361. & 383 & $-2-11$ & 2 & 985 & 1006 & -10 & -8 & 2 & 399 & 412 & -8 & -6 & 2 & 1061 & 1061 & -5 & -4 & 2 & 2194 & 2210 \\
\hline$-5-15$ & 2 & 401 & 368 & $-1-11$ & 2 & 418 & 453 & -9 & -8 & 2 & 297 & 301 & -7 & -6 & 2 & 663 & 674 & -4 & -4 & 2 & 256 & 256 \\
\hline$-3-15$ & 2 & 379 & 364 & $0-11$ & 2 & 987 & 961 & $-B$ & -8 & 2 & 687 & 694 & -6 & -6 & 2 & 1093 & 1129 & -3 & -4 & 2 & 2136 & 2074 \\
\hline$-1-15$ & 2 & 239 & 265 & $1-11$ & 2 & 515 & 522 & -6 & -8 & 2 & 996 & 1019 & -5 & -6 & 2 & 1690 & 1656 & -2 & -4 & 2 & 1626 & 1559 \\
\hline$-7-14$ & 2 & 310 & 314 & $2-11$ & 2 & 630 & 611 & -4 & -8 & 2 & 193 & 191 & -4 & -6 & 2 & 1519 & 1525 & -1 & -4 & & 1674 & 1657 \\
\hline $0-14$ & 2 & 495 & 462 & $3-11$ & 2 & 268 & 260 & -3 & -8 & 2 & 384 & 366 & -3 & -6 & 2 & 1112 & 1090 & 0 & -4 & 2 & 2132 & 2062 \\
\hline$-9-13$ & 2 & 502 & 492 & $5-11$ & 2 & 356 & 389 & -2 & -8 & 2 & 266 & 319 & -2 & -6 & 2 & 537 & 470 & 1 & -4 & 2 & 2216 & 2180 \\
\hline$-8-13$ & 2 & 216 & 234 & $-6-10$ & 2 & 999 & 1041 & 0 & -8 & 2 & 178 & 171 & -1 & -6 & 2 & 1658 & 1777 & 2 & -4 & 2 & 1960 & 1958 \\
\hline$-7-13$ & 2 & 578 & 558 & $-5-10$ & 2 & 208 & 216 & $I$ & -8 & 2 & 1054 & 1046 & 0 & -6 & 2 & 456 & 439 & 3 & -4 & & 1149 & 115 \\
\hline
\end{tabular}

observed and calculated structure factors: $\left[\mathrm{OS}(\mathrm{CO})_{2} \mathrm{PPH}_{3}\right]_{2}$ (MU-I) (MH-PPH$)_{2}$ )

Page 6

h $k 1$ 10FO 10FC

$\begin{array}{lllll}4 & -4 & 2 & 980 & 984\end{array}$

$\begin{array}{lllll}5 & -4 & 2 & 273 & 275\end{array}$

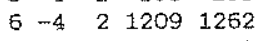

$7 \rightarrow 4 \quad 2 \quad 288 \quad 306$

$\begin{array}{lllll}8 & -4 & 2 & 832 & 817\end{array}$

$\begin{array}{lllll}-12 & -3 & 2 & 512 & 484\end{array}$

$\begin{array}{lllll}-11 & -3 & 2 & 429 & 411\end{array}$

$\begin{array}{lllll}-10 & -3 & 2 & 416 & 456\end{array}$

$\begin{array}{lllll}-9 & -3 & 2 & 342 & 361\end{array}$

$\begin{array}{lllll}-7 & -3 & 2 & 865 & 864\end{array}$

$\begin{array}{lllll}-6 & -3 & 2 & 620 & 645\end{array}$

$\begin{array}{lllll}-5 & -3 & 2 & 195 & 154\end{array}$

$\begin{array}{llllll}-4 & -3 & 2 & 744 & 750\end{array}$

$\begin{array}{lllll}-3 & -3 & 2 & 1393 & 1357\end{array}$

$\begin{array}{llllll}-2 & -3 & 2 & 1418 & 1355\end{array}$

$\begin{array}{lllll}-1 & -3 & 2 & 1985 & 1876\end{array}$

$\begin{array}{lllll}0 & -3 & 2 & 1104 & 1082\end{array}$

$\begin{array}{lllll}1 & -3 & 2 & 372 & 342\end{array}$

$\begin{array}{lllll}2 & -3 & 2 & 2635 & 2653\end{array}$

$\begin{array}{lllll}3 & -3 & 2 & 598 & 610\end{array}$

$\begin{array}{lllll}4 & -3 & 2 & 1491 & 1537\end{array}$

$\begin{array}{lllll}5 & -3 & 2 & 326 & 328\end{array}$

$\begin{array}{lllll}6 & -3 & 2 & 1526 & 1560\end{array}$

$\begin{array}{lllll}7 & -3 & 2 & 720 & 797\end{array}$

$\begin{array}{lllll}8 & -3 & 2 & 1173 & 1187\end{array}$

$\begin{array}{lllll}9 & -3 & 2 & 385 & 415\end{array}$

$\begin{array}{lllll}-12 & -2 & 2 & 546 & 556\end{array}$

$\begin{array}{llllll}-11 & -2 & 2 & 744 & 749\end{array}$

$\begin{array}{lllll}-10 & -2 & 2 & 687 & 701\end{array}$

$\begin{array}{lllll}-9 & -2 & 2 & 653 & 630\end{array}$

$\begin{array}{lllll}-7 & -2 & 2 & 1042 & 1031\end{array}$

$\begin{array}{lllll}-6 & -2 & 2 & 390 & 395\end{array}$

$\begin{array}{llllll}-5 & -2 & 2 & 1198 & 1193\end{array}$

$\begin{array}{lllll}-4 & -2 & 2 & 785 & 777\end{array}$ h $k 1$ 10FO 1OFC

$\begin{array}{lllll}-2 & -2 & 2 & 1202 & 1126\end{array}$

$\begin{array}{lllll}-1 & -2 & 2 & 241 & 252\end{array}$

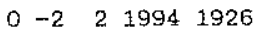

$\begin{array}{lllll}1 & -2 & 2 & 1425 & 1398\end{array}$

$\begin{array}{lllll}2 & -2 & 2 & 1279 & 1302\end{array}$

$\begin{array}{lllll}3 & -2 & 2 & 136 & 128\end{array}$

$\begin{array}{llllll}4 & -2 & 2 & 1228 & 1243\end{array}$

$\begin{array}{lllll}5 & -2 & 2 & 476 & 462\end{array}$

$\begin{array}{lllll}6 & -2 & 2 & 1092 & 1123\end{array}$

$\begin{array}{lllll}7 & -2 & 2 & 1045 & 1063\end{array}$

$\begin{array}{lllll}8 & -2 & 2 & 1058 & 1077\end{array}$

$\begin{array}{lllll}9 & -2 & 2 & 735 & 743\end{array}$

$\begin{array}{lllll}10 & -2 & 2 & 438 & 461\end{array}$

$\begin{array}{lllll}11 & -2 & 2 & 574 & 558\end{array}$

$\begin{array}{lllll}-12 & -1 & 2 & 280 & 276\end{array}$

$\begin{array}{lllll}-11 & -1 & 2 & 1021 & 1051\end{array}$

$\begin{array}{lllll}-10 & -1 & 2 & 537 & 549\end{array}$

$\begin{array}{lllll}-9 & -1 & 2 & 1080 & 1099\end{array}$

$\begin{array}{lllll}-8 & -1 & 2 & 207 & 199\end{array}$

$\begin{array}{llllll}-7 & -1 & 2 & 1111 & 1133\end{array}$

$\begin{array}{lllll}-6 & -1 & 2 & 1082 & 1086\end{array}$

$\begin{array}{lllll}-5 & -1 & 2 & 1866 & 1899\end{array}$

$\begin{array}{lllll}-4 & -1 & 2 & 671 & 661\end{array}$

$\begin{array}{lllll}-3 & -1 & 2 & 4.16 & 413\end{array}$

$\begin{array}{lllll}-2 & -1 & 2 & 277 & 258\end{array}$

$\begin{array}{lllll}-1 & -1 & 2 & 147 & 148\end{array}$

$\begin{array}{lllll}0 & -1 & 2 & 1059 & 1039\end{array}$

$\begin{array}{lllll}1 & -1 & 2 & 397 & 328\end{array}$

$\begin{array}{lllll}2 & -1 & 2 & 840 & 841\end{array}$

$\begin{array}{lllll}4 & -1 & 2 & 700 & 710\end{array}$

$\begin{array}{lllll}5 & -1 & 2 & 173 & 1.45\end{array}$

$\begin{array}{lllll}7 & -1 & 2 & 777 & 788\end{array}$

$\begin{array}{lllll}8 & -1 & 2 & 229 & 240\end{array}$

$\begin{array}{lllll}9 & -1 & 2 & 892 & 913\end{array}$ $\mathrm{h} \mathrm{k}$ l. 10FO 10FC

$\begin{array}{lllll}10 & -1 & 2 & 329 & 367\end{array}$

$\begin{array}{lllll}11 & -1 & 2 & 400 & 377\end{array}$

$\begin{array}{lllll}-11 & 0 & 2 & 743 & 765\end{array}$

$\begin{array}{lllll}-9 & 0 & 2 & 1082 & 1085\end{array}$

$\begin{array}{lllll}-7 & 0 & 2 & 733 & 755\end{array}$

$\begin{array}{lllll}-6 & 0 & 2 & 1523 & 1520\end{array}$

$\begin{array}{lllll}-5 & 0 & 2 & 851 & 867\end{array}$

$\begin{array}{llllll}-4 & 0 & 2 & 2546 & 2527\end{array}$

$\begin{array}{lllll}-3 & 0 & 2 & 1927 & 1877\end{array}$

$\begin{array}{llllll}-2 & 0 & 2 & 1766 & 1735\end{array}$

$\begin{array}{lllll}-1 & 0 & 2 & 327 & 312\end{array}$

$\begin{array}{lllll}0 & 0 & 2 & 2497 & 2604\end{array}$

$\begin{array}{lllll}1 & 0 & 2 & 166 & 122\end{array}$

$\begin{array}{lllll}2 & 0 & 2 & 1248 & 1170\end{array}$

$\begin{array}{lllll}3 & 0 & 2 & 541 & 547\end{array}$

$4002540 \quad 525$

$5 \quad 0 \quad 2 \quad 1406 \quad 1455$

$\begin{array}{lllll}6 & 0 & 2 & 326 & 333\end{array}$

$\begin{array}{lllll}7 & 0 & 2 & 315 & 332\end{array}$

$\begin{array}{lllll}8 & 0 & 2 & 394 & 430\end{array}$

$\begin{array}{lllll}9 & 0 & 2 & 593 & 612\end{array}$

$\begin{array}{lllll}11 & 0 & 2 & 250 & 258\end{array}$

$\begin{array}{lllll}-10 & 1 & 2 & 308 & 272\end{array}$

$\begin{array}{lllll}-9 & 1 & 2 & 686 & 661\end{array}$

$\begin{array}{lllll}-8 & 1 & 2 & 212 & 113\end{array}$

$\begin{array}{lllll}-7 & 1 & 2 & 446 & 447\end{array}$

$\begin{array}{lllll}-6 & 1 & 2 & 1124 & 11.07\end{array}$

$\begin{array}{lllll}-5 & 1 & 2 & 410 & 403\end{array}$

$\begin{array}{lllll}-4 & 1 & 2 & 2492 & 2445\end{array}$

$\begin{array}{lllll}-3 & 1 & 2 & 1363 & 1309\end{array}$

$\begin{array}{lllll}-2 & 1 & 2 & 2430 & 2325\end{array}$

$\begin{array}{lllll}-1 & 1 & 2 & 1403 & 1317\end{array}$

$\begin{array}{lllll}0 & 1 & 2 & 3163 & 3327\end{array}$

$1121544 \quad 1511$ h $k 1$ 10Fo 10FC

$\begin{array}{lllll}2 & 1 & 2 & 2451 & 2508\end{array}$

$\begin{array}{lllll}3 & 1 & 2 & 883 & 871\end{array}$

$\begin{array}{lllll}4 & 1 & 2 & 540 & 544\end{array}$

$\begin{array}{lllll}5 & 1 & 2 & 1562 & 1621\end{array}$

$\begin{array}{lllll}6 & 1 & 2 & 249 & 242\end{array}$

$\begin{array}{lllll}7 & 1 & 2 & 1490 & 1557\end{array}$

$\begin{array}{lllll}8 & 1 & 2 & 467 & 456\end{array}$

$\begin{array}{lllll}9 & 1 & 2 & 282 & 278\end{array}$

$\begin{array}{lllll}-11 & 2 & 2 & 247 & 279\end{array}$

$\begin{array}{lllll}-9 & 2 & 2 & 250 & 276\end{array}$

$\begin{array}{lllll}-6 & 2 & 2 & 356 & 338\end{array}$

$\begin{array}{lllll}-5 & 2 & 2 & 397 & 417\end{array}$

$\begin{array}{lllll}-4 & 2 & 2 & 2111 & 2098\end{array}$

$\begin{array}{lllll}-3 & 2 & 2 & 359 & 373\end{array}$

$\begin{array}{lllll}-2 & 2 & 2 & 2717 & 2630\end{array}$

$\begin{array}{lllll}-1 & 2 & 2 & 1092 & 1033\end{array}$

o 2 216441614

$\begin{array}{llllll}1 & 2 & 2 & 2217 & 2.162\end{array}$

$\begin{array}{llllll}2 & 2 & 2 & 1267 & 1251\end{array}$

$\begin{array}{lllll}3 & 2 & 2 & 1159 & 1196\end{array}$

$\begin{array}{lllll}4 & 2 & 2 & 1442 & 1466\end{array}$

$\begin{array}{lllll}5 & 2 & 2 & 1329 & 1371\end{array}$

$\begin{array}{lllll}6 & 2 & 2 & 847 & 850\end{array}$

$\begin{array}{llllll}7 & 2 & 2 & 1853 & 1859\end{array}$

$\begin{array}{lllll}8 & 2 & 2 & 491 & 499\end{array}$

$\begin{array}{lllll}9 & 2 & 2 & 1045 & 1032\end{array}$

$\begin{array}{lllll}10 & 2 & 2 & 253 & 306\end{array}$

$\begin{array}{lllll}11 & 2 & 2 & 311 & 327\end{array}$

$\begin{array}{lllll}-10 & 3 & 2 & 381 & 385\end{array}$

$\begin{array}{lllll}-8 & 3 & 2 & 286 & 271\end{array}$

$\begin{array}{lllll}-6 & 3 & 2 & 658 & 654\end{array}$

$\begin{array}{lllll}-5 & 3 & 2 & 298 & 304\end{array}$

$\begin{array}{lllll}-4 & 3 & 2 & 692 & 709\end{array}$

$\begin{array}{lllll}-2 & 3 & 2 & 1483 & 1407\end{array}$ h $\mathrm{k} 1$ 10Fo 10FC

$\begin{array}{lllll}-1 & 3 & 2 & 684 & 666\end{array}$

- 32214131266

$\begin{array}{lllll}1 & 3 & 2 & 1797 & 1758\end{array}$

$\begin{array}{lllll}2 & 3 & 2 & 1429 & 1443\end{array}$

$\begin{array}{lllll}3 & 3 & 2 & 2328 & 2329\end{array}$

$\begin{array}{lllll}4 & 3 & 2 & 807 & 817\end{array}$

$\begin{array}{lllll}5 & 3 & 2 & 770 & 813\end{array}$

$\begin{array}{lllll}6 & 3 & 2 & 808 & 825\end{array}$

$\begin{array}{lllll}7 & 3 & 2 & 1240 & 1294\end{array}$

$8 \quad 3 \quad 2 \quad 1187 \quad 1225$

$\begin{array}{lllll}9 & 3 & 2 & 1176 & 1199\end{array}$

$\begin{array}{lllll}10 & 3 & 2 & 889 & 894\end{array}$

$\begin{array}{lllll}12 & 3 & 2 & 634 & 625\end{array}$

$\begin{array}{lllll}-10 & 4 & 2 & 645 & 674\end{array}$

$\begin{array}{lllll}-9 & 4 & 2 & 264 & 290\end{array}$

$\begin{array}{lllll}-8 & 4 & 2 & 648 & 636\end{array}$

$\begin{array}{lllll}-7 & 4 & 2 & 294 & 310\end{array}$

$\begin{array}{lllll}-6 & 4 & 2 & 1634 & 1651\end{array}$

$\begin{array}{lllll}-4 & 4 & 2 & 1265 & 1266\end{array}$

$\begin{array}{lllll}-3 & 4 & 2 & 182 & 156\end{array}$

$\begin{array}{lllll}-2 & 4 & 2 & 592 & 582\end{array}$

$\begin{array}{lllll}-1 & 4 & 2 & 1478 & 1445\end{array}$

$\begin{array}{lllll}0 & 4 & 2 & 234 & 228\end{array}$

$142879 \quad 865$

$\begin{array}{lllll}24 & 2 & 1298 & 1295\end{array}$

34218431855

542861895

$\begin{array}{lllll}6 & 4 & 2 & 714 & 741\end{array}$

$\begin{array}{lllll}7 & 4 & 2 & 850 & 888\end{array}$

84214001402

942901944

$\begin{array}{lllll}10 \quad 4 \quad 2 & 992 & 982\end{array}$

$\begin{array}{lllll}12 & 4 & 2 & 723 & 704\end{array}$

$\begin{array}{lllll}-9 & 5 & 2 & 291 & 288\end{array}$ 


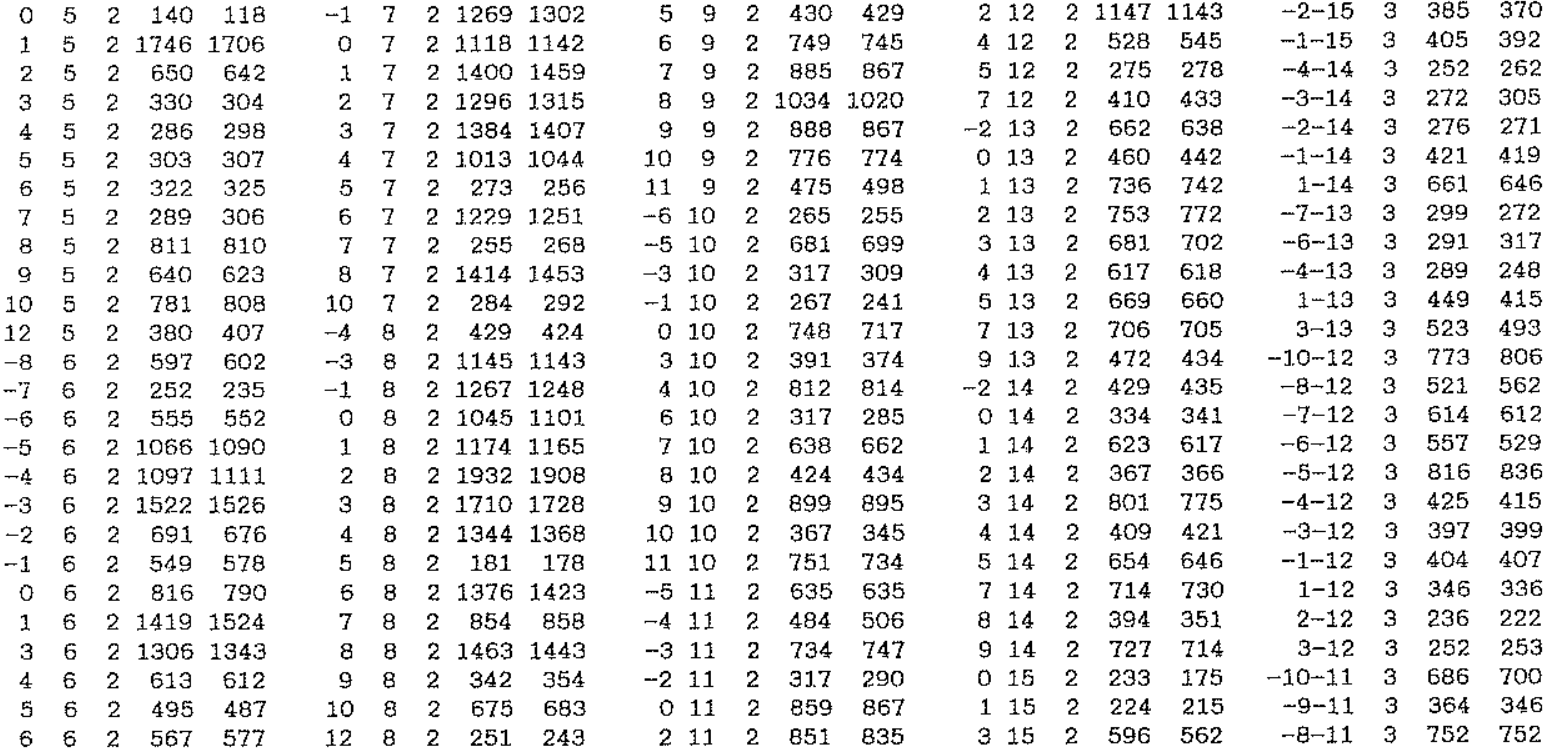

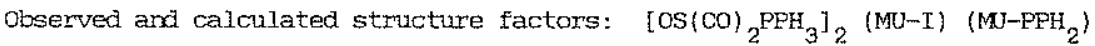

Page 8

$\mathrm{h} k 1$ 10FO 10FC

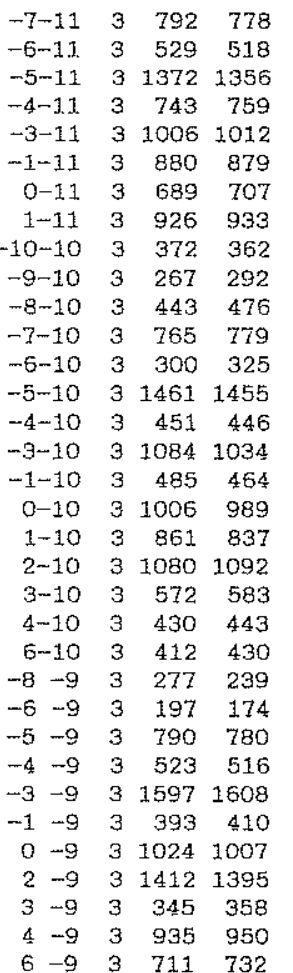

h $\mathrm{k} \quad 1$ 10FO $10 \mathrm{FC}$

$\begin{array}{lllll}7 & -9 & 3 & 321 & 304\end{array}$

$\begin{array}{lllll}-12 & -8 & 3 & 503 & 499\end{array}$

$\begin{array}{lllll}-11 & -8 & 3 & 448 & 474\end{array}$

$\begin{array}{lllll}-10 & -8 & 3 & 570 & 545\end{array}$

$\begin{array}{lllll}-7 & -8 & 3 & 594 & 594\end{array}$

$\begin{array}{lllll}-5 & -8 & 3 & 399 & 385\end{array}$

$\begin{array}{lllll}-4 & -8 & 3 & 579 & 576\end{array}$

$\begin{array}{lllll}-3 & -8 & 3 & 1006 & 960\end{array}$

$\begin{array}{lllll}-2 & -8 & 3 & 493 & 510\end{array}$

$\begin{array}{lllll}-1 & -8 & 3 & 401 & 435\end{array}$

$\begin{array}{lllll}0 & -8 & 3 & 676 & 667\end{array}$

$\begin{array}{lllll}2 & -8 & 3 & 1515 & 1492\end{array}$

$\begin{array}{lllll}4 & -8 & 3 & 1171 & 1175\end{array}$

$\begin{array}{lllll}5 & -8 & 3 & 358 & 348\end{array}$

$\begin{array}{lllll}6 & -8 & 3 & 586 & 606\end{array}$

$\begin{array}{lllll}7 & -8 & 3 & 631 & 628\end{array}$

$\begin{array}{lllll}8 & -8 & 3 & 532 & 525\end{array}$

$\begin{array}{lllll}-12 & -7 & 3 & 421 & 461\end{array}$

$\begin{array}{lllll}-11 & -7 & 3 & 871 & 892\end{array}$

$\begin{array}{lllll}-10 & -7 & 3 & 665 & 648\end{array}$

$\begin{array}{lllll}-9 & -7 & 3 & 634 & 629\end{array}$

$\begin{array}{llllll}-7 & -7 & 3 & 477 & 476\end{array}$

$\begin{array}{llllll}-6 & -7 & 3 & 788 & 796\end{array}$

$\begin{array}{lllll}-5 & -7 & 3 & 1154 & 1201\end{array}$

$\begin{array}{lllll}-4 & -7 & 3 & 191 & 200\end{array}$

$\begin{array}{lllll}-3 & -7 & 3 & 178 & 171\end{array}$

$\begin{array}{lllll}-2 & -7 & 3 & 458 & 472\end{array}$

$\begin{array}{lllll}-1 & -7 & 3 & 530 & 534\end{array}$

$\begin{array}{llll}-7 & 3 & 343 & 335\end{array}$

$\begin{array}{lllll}1 & -7 & 3 & 232 & 209\end{array}$

$\begin{array}{lllll}2 & -7 & 3 & 1046 & 1019\end{array}$

$\begin{array}{lllll}3 & -7 & 3 & 308 & 257\end{array}$

$\begin{array}{lllll}4 & -7 & 3 & 1021 & 1046\end{array}$

$\begin{array}{llllll}6 & -7 & 3 & 253 & 288\end{array}$ h $\mathrm{k} 1$ 10FO 1OFC

$\begin{array}{lllll}7 & -7 & 3 & 695 & 680\end{array}$

$\begin{array}{lllll}8 & -7 & 3 & 260 & 219\end{array}$

$\begin{array}{lllll}-11 & -6 & 3 & 859 & 872\end{array}$

$\begin{array}{lllll}-9 & -6 & 3 & 1023 & 1053\end{array}$

$\begin{array}{lllll}-7 & -6 & 3 & 336 & 297\end{array}$

$\begin{array}{lllll}-6 & -6 & 3 & 1345 & 1359\end{array}$

$\begin{array}{lllll}-5 & -6 & 3 & 932 & 935\end{array}$

$\begin{array}{lllll}-4 & -6 & 3 & 1755 & 1743\end{array}$

$\begin{array}{lllll}-3 & -6 & 3 & 529 & 546\end{array}$

$\begin{array}{lllll}-2 & -6 & 3 & 1393 & 1381\end{array}$

$\begin{array}{lllll}-1 & -6 & 3 & 227 & 217\end{array}$

$\begin{array}{lllll}0 & -6 & 3 & 1657 & 1581\end{array}$

$\begin{array}{lllll}1 & -6 & 3 & 381 & 343\end{array}$

$\begin{array}{lllll}2 & -6 & 3 & 259 & 245\end{array}$

$\begin{array}{lllll}3 & -6 & 3 & 527 & 532\end{array}$

$\begin{array}{lllll}4 & -6 & 3 & 563 & 572\end{array}$

$\begin{array}{lllll}5 & -6 & 3 & 219 & 219\end{array}$

$\begin{array}{lllll}7 & -6 & 3 & 200 & 173\end{array}$

$\begin{array}{lllll}9 & -6 & 3 & 667 & 641\end{array}$

$\begin{array}{lllll}-11 & -5 & 3 & 483 & 496\end{array}$

$\begin{array}{lllll}-10 & -5 & 3 & 401 & 438\end{array}$

$\begin{array}{lllll}-9 & -5 & 3 & 1287 & 1301\end{array}$

$\begin{array}{lllll}-8 & -5 & 3 & 657 & 633\end{array}$

$\begin{array}{lllll}-7 & -5 & 3 & 586 & 609\end{array}$

$\begin{array}{lllll}-6 & -5 & 3 & 1602 & 1639\end{array}$

$\begin{array}{lllll}-5 & -5 & 3 & 370 & 355\end{array}$

$\begin{array}{lllll}-4 & -5 & 3 & 2307 & 2340\end{array}$

$\begin{array}{lllll}-3 & -5 & 3 & 799 & 778\end{array}$

$\begin{array}{lllll}-2 & -5 & 3 & 1682 & 1653\end{array}$

$\begin{array}{lllll}-1 & -5 & 3 & 473 & 464\end{array}$

o $-5 \quad 3 \quad 1914 \quad 1890$

$\begin{array}{lllll}1 & -5 & 3 & 1102 & 1070\end{array}$

$\begin{array}{lllll}2 & -5 & 3 & 1467 & 1490\end{array}$

$\begin{array}{llllll}5 & -5 & 3 & 499 & 522\end{array}$ h $k$ I 10FO 10FC

$\begin{array}{lllll}7 & -5 & 3 & 399 & 394\end{array}$ $\begin{array}{lllll}8 & -5 & 3 & 409 & 404\end{array}$ $\begin{array}{lllll}-10 & -4 & 3 & 343 & 320\end{array}$ $\begin{array}{lllll}-9 & -4 & 3 & 527 & 541\end{array}$ $\begin{array}{lllll}-8 & -4 & 3 & 696 & 644\end{array}$ $\begin{array}{lllll}-7 & -4 & 3 & 487 & 450\end{array}$ $\begin{array}{lllll}-6 & -4 & 3 & 1592 & 1577\end{array}$ $\begin{array}{lllll}-5 & -4 & 3 & 266 & 280\end{array}$ $\begin{array}{lllll}-4 & -4 & 3 & 2558 & 2579\end{array}$ $\begin{array}{lllll}-3 & -4 & 3 & 61.5 & 599\end{array}$ $\begin{array}{lllll}-2 & -4 & 3 & 2478 & 2394\end{array}$ $\begin{array}{lllll}-1 & -4 & 3 & 187 & 123\end{array}$

$\begin{array}{lllll}0 & -4 & 3 & 1473 & 1431\end{array}$

$\begin{array}{lllll}1 & -4 & 3 & 2429 & 2396\end{array}$

$\begin{array}{lllll}2 & -4 & 3 & 1510 & 1518\end{array}$

$\begin{array}{lllll}3 & -4 & 3 & 1512 & 1557\end{array}$

$\begin{array}{lllll}4 & -4 & 3 & 528 & 539\end{array}$

$\begin{array}{lllll}5 & -4 & 3 & 739 & 748\end{array}$

$\begin{array}{lllll}6 & -4 & 3 & 523 & 527\end{array}$

$\begin{array}{lllll}7 & -4 & 3 & 621 & 624\end{array}$

$\begin{array}{lllll}8 & -4 & 3 & 199 & 169\end{array}$

$\begin{array}{lllll}9 & -4 & 3 & 332 & 342\end{array}$

$\begin{array}{lllll}-12 & -3 & 3 & 269 & 300\end{array}$

$\begin{array}{lllll}-11 & -3 & 3 & 384 & 391\end{array}$

$\begin{array}{lllll}-7 & -3 & 3 & 279 & 265\end{array}$

$\begin{array}{lllll}-6 & -3 & 3 & 165 & 149\end{array}$

$\begin{array}{lllll}-5 & -3 & 3 & 266 & 299\end{array}$

$\begin{array}{lllll}-4 & -3 & 3 & 1345 & 1332\end{array}$

$\begin{array}{lllll}-3 & -3 & 3 & 224 & 219\end{array}$

$\begin{array}{lllll}-2 & -3 & 3 & 2393 & 2328\end{array}$

$\begin{array}{lllll}-1 & -3 & 3 & 704 & 715\end{array}$

$\begin{array}{llll}-3 & 3 & 506 & 465\end{array}$

$\begin{array}{lllll}1 & -3 & 3 & 2152 & 2107\end{array}$

$\begin{array}{lllll}2 & -3 & 3 & 140 & 106\end{array}$ $h \mathrm{k}$ I 1OEO 1OFC

$\begin{array}{lllll}3 & -3 & 3 & 2673 & 2687\end{array}$

$\begin{array}{lllll}4 & -3 & 3 & 454 & 455\end{array}$

$\begin{array}{lllll}5 & -3 & 3 & 1308 & 1328\end{array}$

$\begin{array}{lllll}6 & -3 & 3 & 671 & 672\end{array}$

$\begin{array}{lllll}7 & -3 & 3 & 886 & 899\end{array}$

$\begin{array}{lllll}8 & -3 & 3 & 705 & 714\end{array}$

$\begin{array}{lllll}9 & -3 & 3 & 623 & 623\end{array}$

$\begin{array}{lllll}10 & -3 & 3 & 443 & 405\end{array}$

$\begin{array}{lllll}-12 & -2 & 3 & 599 & 585\end{array}$

$\begin{array}{lllll}-11 & -2 & 3 & 365 & 390\end{array}$

$\begin{array}{lllll}-10 & -2 & 3 & 619 & 657\end{array}$

$\begin{array}{lllll}-9 & -2 & 3 & 345 & 341\end{array}$

$\begin{array}{lllll}-8 & -2 & 3 & 526 & 513\end{array}$

$\begin{array}{lllll}-7 & -2 & 3 & 285 & 294\end{array}$

$\begin{array}{lllll}-6 & -2 & 3 & 848 & 867\end{array}$

$\begin{array}{lllll}-4 & -2 & 3 & 309 & 316\end{array}$

$\begin{array}{lllll}-3 & -2 & 3 & 933 & 914\end{array}$

$\begin{array}{lllll}-2 & -2 & 3 & 862 & 820\end{array}$

$\begin{array}{lllll}0 & -2 & 3 & 664 & 630\end{array}$

$\begin{array}{lllll}1 & -2 & 3 & 2161 & 1999\end{array}$

$\begin{array}{lllll}2 & -2 & 3 & 1732 & 1709\end{array}$

$\begin{array}{llllll}3 & -2 & 3 & 2115 & 2115\end{array}$

$\begin{array}{llllll}5 & -2 & 3 & 1561 & 1626\end{array}$

$\begin{array}{lllll}5 & -2 & 3 & 669 & 696\end{array}$

$\begin{array}{lllll}7 & -2 & 3 & 902 & 909\end{array}$

$\begin{array}{lllll}8 & -2 & 3 & 1272 & 1308\end{array}$

$\begin{array}{lllll}9 & -2 & 3 & 640 & 647\end{array}$

$\begin{array}{lllll}10 & -2 & 3 & 716 & 698\end{array}$

$\begin{array}{lllll}-11 & -1 & 3 & 360 & 341\end{array}$

$\begin{array}{lllll}-10 & -1 & 3 & 1045 & 1046\end{array}$

$\begin{array}{lllll}-9 & -1 & 3 & 430 & 470\end{array}$

$\begin{array}{lllll}-8 & -1 & 3 & 828 & 850\end{array}$

$\begin{array}{lllll}-7 & -1 & 3 & 428 & 449\end{array}$

$\begin{array}{lllll}-6 & -1 & 3 & 995 & 997\end{array}$ 
$\begin{array}{lllll}-2 & 3 & 3 & 1079 & 1018\end{array}$

13211276

$\begin{array}{lllll}1 & 1 & 3 & 2254 & 2179\end{array}$

$\begin{array}{llllll}2 & 1 & 3 & 2542 & 2573\end{array}$

$\begin{array}{lllll}3 & 1 & 3 & 1575 & 1590\end{array}$

$\begin{array}{lllll}4 & 1 & 3 & 419 & 394\end{array}$

$\begin{array}{lllll}5 & 1 & 3 & 595 & 581\end{array}$

$\begin{array}{lllll}6 & 1 & 3 & 995 & 1033\end{array}$

$813540 \quad 513$

$\begin{array}{lllll}9 & 1 & 3 & 407 & 415\end{array}$

$\begin{array}{lllll}10 & 1 & 3 & 384 & 394\end{array}$

$\begin{array}{lllll}-9 & 2 & 3 & 404 & 395\end{array}$

$\begin{array}{llllll}-8 & 2 & 3 & 538 & 546\end{array}$

$\begin{array}{lllll}-7 & 2 & 3 & 229 & 239\end{array}$

$\begin{array}{lllll}-5 & 2 & 3 & 1080 & 1104\end{array}$

$\begin{array}{lllll}-4 & 2 & 3 & 423 & 437\end{array}$

$\begin{array}{lllll}-3 & 2 & 3 & 2907 & 2875\end{array}$

$\begin{array}{lllll}-2 & 2 & 3 & 446 & 425\end{array}$

$\begin{array}{llllll}-1 & 2 & 3 & 1351 & 1308\end{array}$

o $22 \quad 321902164$

$\begin{array}{llllll}1 & 2 & 3 & 1513 & 1454\end{array}$

$\begin{array}{lllll}2 & 2 & 3 & 2323 & 2288\end{array}$

$\begin{array}{llllll}3 & 2 & 3 & 1639 & 1631\end{array}$

$\begin{array}{llllll}4 & 2 & 3 & 1477 & 1536\end{array}$

$\begin{array}{lllll}5 & 2 & 3 & 137 & 121\end{array}$

$\begin{array}{lllll}6 & 2 & 3 & 1396 & 1418\end{array}$ $\begin{array}{llllll}-1 & 3 & 3 & 1516 & 1456\end{array}$

$\begin{array}{lllll}0 & 3 & 3 & 319 & 310\end{array}$

$\begin{array}{lllll}1 & 3 & 3 & 573 & 538\end{array}$

$\begin{array}{llllll}2 & 3 & 3 & 3656 & 3679\end{array}$

$\begin{array}{lllll}3 & 3 & 3 & 501 & 522\end{array}$

$\begin{array}{llllll}4 & 3 & 3 & 2132 & 2191\end{array}$

$\begin{array}{lllll}5 & 3 & 3 & 184 & 171\end{array}$

$\begin{array}{llllll}6 & 3 & 3 & 1134 & 1166\end{array}$

$\begin{array}{llllll}7 & 3 & 3 & 1217 & 1234\end{array}$

$\begin{array}{llllll}8 & 3 & 3 & 1366 & 1392\end{array}$

$\begin{array}{lllll}9 & 3 & 3 & 859 & 830\end{array}$

$\begin{array}{lllll}10 & 3 & 3 & 660 & 652\end{array}$

$\begin{array}{lllll}11 & 3 & 3 & 326 & 330\end{array}$

$\begin{array}{lllll}-10 & 4 & 3 & 325 & 324\end{array}$

$\begin{array}{lllll}-9 & 4 & 3 & 642 & 647\end{array}$

$\begin{array}{lllll}-8 & 4 & 3 & 261 & 249\end{array}$

$\begin{array}{llllll}-7 & 4 & 3 & 374 & 370\end{array}$

$\begin{array}{lllll}-5 & 4 & 3 & 922 & 941\end{array}$

$\begin{array}{lllll}-3 & 4 & 3 & 264 & 260\end{array}$

$\begin{array}{lllll}-2 & 4 & 3 & 168 & 163\end{array}$

$-1 \quad 4 \quad 3 \quad 1563 \quad 1504$

$\begin{array}{lllll}0 & 4 & 3 & 268 & 234\end{array}$

$\begin{array}{lllll}1 & 4 & 3 & 195 & 178\end{array}$

24310751020 $\begin{array}{lllll}12 & 4 & 3 & 223 & 189\end{array}$

$\begin{array}{lllll}-9 & 5 & 3 & 788 & 822\end{array}$

$\begin{array}{lllll}-1 & 5 & 3 & 571 & 557\end{array}$

$\begin{array}{lllll}-7 & 5 & 3 & 571 & 557 \\ -5 & 5 & 3 & 568 & 587\end{array}$

$\begin{array}{lllll}-5 & 5 & 3 & 1162 & 1187\end{array}$

$\begin{array}{lllll}-4 & 5 & 3 & 995 & 1003\end{array}$

$\begin{array}{lllll}-3 & 5 & 3 & 1004 & 998\end{array}$

$\begin{array}{lllll}-1 & 5 & 3 & 213 & 220\end{array}$

$\begin{array}{lllll}0 & 5 & 3 & 1110 & 1075\end{array}$

$\begin{array}{lllll}1 & 5 & 3 & 168 & 179\end{array}$

$\begin{array}{lllll}2 & 5 & 3 & 924 & 891\end{array}$

$\begin{array}{lllll}3 & 5 & 3 & 1681 & 1637\end{array}$

$\begin{array}{lllll}5 & 3 & 1075 & 1089\end{array}$

$\begin{array}{lllll}5 & 5 & 3 & 870 & 897\end{array}$

$\begin{array}{lllll}6 & 5 & 3 & 941 & 923\end{array}$

$\begin{array}{lllll}7 & 5 & 3 & 689 & 704\end{array}$

$\begin{array}{lllll}8 & 5 & 3 & 484 & 488\end{array}$

$\begin{array}{lllll}9 & 5 & 3 & 949 & 994\end{array}$

$\begin{array}{lllll}10 & 5 & 3 & 211 & 205\end{array}$

$\begin{array}{lllll}11 & 5 & 3 & 833 & 794\end{array}$

$\begin{array}{lllll}12 & 5 & 3 & 307 & 300\end{array}$

$\begin{array}{lllll}-9 & 6 & 3 & 823 & 814\end{array}$

$\begin{array}{lllll}-7 & 6 & 3 & 668 & 643\end{array}$

$\begin{array}{lllll}-6 & 6 & 3 & 413 & 408\end{array}$

$\begin{array}{lllll}-5 & 6 & 3 & 590 & 577\end{array}$ $\begin{array}{llll}6 & 3 & 275 & 263\end{array}$

$\begin{array}{lllll}9 & 6 & 3 & 653 & 649\end{array}$

$\begin{array}{lllll}1 & 6 & 3 & 586 & 589\end{array}$

$\begin{array}{lllll}7 & 7 & 3 & 565 & 571\end{array}$

$\begin{array}{lllll}-6 & 7 & 3 & 349 & 347\end{array}$

$\begin{array}{llllll}-4 & 7 & 3 & 1423 & 1394\end{array}$

$\begin{array}{llllll}-2 & 7 & 3 & 1522 & 1561\end{array}$

$\begin{array}{lllll}0 & 7 & 3 & 885 & 896\end{array}$

$\begin{array}{llllll}1 & 7 & 3 & 1481 & 1525\end{array}$

$\begin{array}{lllll}7 & 3 & 1257 & 1267\end{array}$

$\begin{array}{llllll}3 & 7 & 3 & 1268 & 1250\end{array}$

$\begin{array}{lllll}4 & 7 & 3 & 746 & 709\end{array}$

$\begin{array}{lllll}5 & 7 & 3 & 554 & 556\end{array}$

$\begin{array}{lllll}6 & 7 & 3 & 325 & 327\end{array}$

$\begin{array}{lllll}7 & 7 & 3 & 955 & 940\end{array}$

$\begin{array}{llllll}8 & 7 & 3 & 184 & 216\end{array}$

$\begin{array}{lllll}9 & 7 & 3 & 224 & 250\end{array}$

$\begin{array}{rrrrr}10 & 7 & 3 & 209 & 144\end{array}$

$\begin{array}{lllll}-7 & 8 & 3 & 319 & 312\end{array}$

$\begin{array}{lllll}-5 & 8 & 3 & 249 & 220\end{array}$

$\begin{array}{llllll}-4 & 8 & 3 & 674 & 687\end{array}$

$\begin{array}{lllll}-3 & 8 & 3 & 519 & 520\end{array}$

$\begin{array}{llllll}-2 & 8 & 3 & 1576 & 1601\end{array}$

$\begin{array}{lllll}-1 & 8 & 3 & 499 & 484\end{array}$

$\begin{array}{lllll}0 & 8 & 3 & 973 & 958\end{array}$

Observed and calculated structure factors: [OS(CO) $\left.{ }_{2} \mathrm{PPH}_{3}\right]_{2}$ (MU-I) (MU-PPH ${ }_{2}$ )

Page 10

$\begin{array}{rrrrr}\mathrm{h} & \mathrm{k} & \mathrm{l} & 10 \mathrm{FO} & 10 \mathrm{Fc} \\ 1 & 8 & 3 & 1268 & 1332 \\ 2 & 8 & 3 & 974 & 944 \\ 3 & 8 & 3 & 1821 & 1820 \\ 4 & 8 & 3 & 876 & 895 \\ 5 & 8 & 3 & 1481 & 1509 \\ 6 & 8 & 3 & 536 & 547 \\ 7 & 8 & 3 & 1188 & 1187 \\ 8 & 8 & 3 & 512 & 525 \\ 9 & 8 & 3 & 657 & 692 \\ -3 & 9 & 3 & 652 & 617 \\ -2 & 9 & 3 & 879 & 849 \\ -1 & 9 & 3 & 549 & 520 \\ 0 & 9 & 3 & 918 & 916 \\ 1 & 9 & 3 & 892 & 888 \\ 2 & 9 & 3 & 397 & 366 \\ 3 & 9 & 3 & 1596 & 1606 \\ 4 & 9 & 3 & 522 & 538 \\ 5 & 9 & 3 & 1375 & 1416 \\ 6 & 9 & 3 & 402 & 398 \\ 7 & 9 & 3 & 893 & 975 \\ 8 & 9 & 3 & 1007 & 1010 \\ 9 & 9 & 3 & 826 & 798 \\ 10 & 9 & 3 & 542 & 565 \\ 11 & 9 & 3 & 409 & 417 \\ 12 & 9 & 3 & 259 & 304 \\ -6 & 10 & 3 & 261 & 238 \\ -5 & 10 & 3 & 282 & 258 \\ -4 & 10 & 3 & 266 & 234 \\ -2 & 10 & 3 & 222 & 194 \\ 0 & 10 & 3 & 541 & 525 \\ 1 & 10 & 3 & 217 & 218 \\ 2 & 10 & 3 & 388 & 403 \\ 3 & 10 & 3 & 939 & 943 \\ 4 & 10 & 3 & 283 & 290\end{array}$

h $k$ I 10FO 10FC

h $k$ l 10Fo 10Fc

$\begin{array}{lllll}5 & 10 & 3 & 936 & 958\end{array}$

$\begin{array}{lllll}7 & 10 & 3 & 350 & 341\end{array}$

$\begin{array}{lllll}8 & 10 & 3 & 952 & 948\end{array}$

$\begin{array}{lllll}9 & 10 & 3 & 334 & 324\end{array}$

$\begin{array}{lllll}10 & 10 & 3 & 1032 & 1023\end{array}$

$\begin{array}{lllll}11 & 10 & 3 & 315 & 320\end{array}$

$\begin{array}{lllll}12 & 10 & 3 & 525 & 525\end{array}$

$\begin{array}{lllll}-5 & 11 & 3 & 479 & 473\end{array}$

$\begin{array}{lllll}-4 & 11 & 3 & 461 & 495\end{array}$

$\begin{array}{lllll}-3 & 11 & 3 & 326 & 327\end{array}$

$\begin{array}{lllll}-2 & 11 & 3 & 323 & 308\end{array}$

$\begin{array}{lllll}-1 & 11 & 3 & 222 & 264\end{array}$

$\begin{array}{lllll}0 & 11 & 3 & 484 & 481\end{array}$

$\begin{array}{lllll}1 & 11 & 3 & 872 & 914\end{array}$

$\begin{array}{lllll}3 & 11 & 3 & 371 & 346\end{array}$

$\begin{array}{lllll}4 & 11 & 3 & 596 & 595\end{array}$

$\begin{array}{lllll}5 & 11 & 3 & 374 & 347\end{array}$

$\begin{array}{lllll}6 & 11 & 3 & 216 & 239\end{array}$

$\begin{array}{lllll}7 & 11 & 3 & 251 & 238\end{array}$

$\begin{array}{lllll}8 & 11 & 3 & 650 & 662\end{array}$

$\begin{array}{lllll}10 & 11 & 3 & 890 & 886\end{array}$

$\begin{array}{lllll}-4 & 12 & 3 & 345 & 356\end{array}$

$\begin{array}{lllll}-3 & 12 & 3 & 812 & 790\end{array}$

$\begin{array}{lllll}-2 & 12 & 3 & 392 & 388\end{array}$

$\begin{array}{lllll}-1 & 12 & 3 & 498 & 517\end{array}$

$\begin{array}{lllll}1 & 12 & 3 & 813 & 810\end{array}$

$\begin{array}{lllll}2 & 12 & 3 & 316 & 317\end{array}$

$\begin{array}{lllll}3 & 12 & 3 & 741 & 746\end{array}$

$\begin{array}{lllll}10 & 12 & 3 & 396 & 386\end{array}$

$\begin{array}{lllll}-3 & 13 & 3 & 738 & 736\end{array}$

$\begin{array}{lllll}-1 & 13 & 3 & 658 & 636\end{array}$

$\begin{array}{lllll}1 & 13 & 3 & 428 & 434\end{array}$

$\begin{array}{lllll}2 & 13 & 3 & 716 & 740\end{array}$

$\begin{array}{lllll}3 & 13 & 3 & 696 & 694\end{array}$ h $\mathrm{k} I$ 10FO 10FC

$\begin{array}{lllll}4 & 13 & 3 & 561 & 567\end{array}$

$\begin{array}{lllll}5 & 13 & 3 & 234 & 291\end{array}$

$\begin{array}{lllll}7 & 13 & 3 & 222 & 219\end{array}$

$\begin{array}{lllll}8 & 13 & 3 & 319 & 311\end{array}$

$\begin{array}{lllll}-2 & 14 & 3 & 303 & 286\end{array}$

$\begin{array}{lllll}-1 & 14 & 3 & 580 & 559\end{array}$

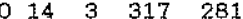

$\begin{array}{lllll}1 & 14 & 3 & 271 & 230\end{array}$

$\begin{array}{lllll}2 & 14 & 3 & 739 & 725\end{array}$

$\begin{array}{lllll}3 & 14 & 3 & 249 & 253\end{array}$

$\begin{array}{lllll}4 & 14 & 3 & 858 & 848\end{array}$

$\begin{array}{lllll}6 & 14 & 3 & 581 & 572\end{array}$

$\begin{array}{lllll}7 & 14 & 3 & 276 & 294\end{array}$

$\begin{array}{lllll}8 & 14 & 3 & 589 & 580\end{array}$

$\begin{array}{lllll}9 & 14 & 3 & 360 & 362\end{array}$

$\begin{array}{lllll}0 & 15 & 3 & 380 & 316\end{array}$

$\begin{array}{lllll}2 & 15 & 3 & 494 & 508\end{array}$

$\begin{array}{lllll}4 & 15 & 3 & 758 & 757\end{array}$

$\begin{array}{lllll}6 & 15 & 3 & 546 & 530\end{array}$

$\begin{array}{lllll}7 & 15 & 3 & 397 & 420\end{array}$

$\begin{array}{lllll}8 & 15 & 3 & 479 & 458\end{array}$

$\begin{array}{lllll}2 & 16 & 3 & 224 & 109\end{array}$

$\begin{array}{lllll}4 & 16 & 3 & 393 & 406\end{array}$

$\begin{array}{lllll}6 & 16 & 3 & 361 & 362\end{array}$

$\begin{array}{llll}-5-15 & 4 & 541 & 532\end{array}$

$-3-15 \quad 4 \quad 666 \quad 645$

$-2-15 \quad 225 \quad 254$

$-4-14 \quad 4 \quad 503 \quad 511$

$\begin{array}{llll}-3-14 \quad 4 \quad 479 \quad 502 & 2\end{array}$

$\begin{array}{llll}-2-14 & 4 & 425 & 474\end{array}$

$\begin{array}{llll}-1-14 & 4 & 321 & 295\end{array}$

$\begin{array}{llll}0-14 & 4 & 575 & 548\end{array}$

$-6-13 \quad 4 \quad 235 \quad 252$

$\begin{array}{lll}-5-13 & 4 & 240 \\ -240\end{array}$ $\mathrm{h} k \mathrm{k}$ 1OFO 10FC

$-4-13 \quad 4 \quad 318 \quad 309$

$\begin{array}{llll}-2-13 \quad 4 & 384 & 395\end{array}$

$0-13 \quad 4 \quad 401 \quad 412$

$-13 \quad 4 \quad 566 \quad 567$

$\begin{array}{llll}-9-12 \quad 4 & 544 & 569\end{array}$

$\begin{array}{llll}-7-12 & 4 & 316 & 320\end{array}$

$\begin{array}{llll}-6-12 & 4 & 717 & 720\end{array}$

$-5-124305306$

$-4-12 \quad 4 \quad 498 \quad 485$

$\begin{array}{llll}-3-12 \quad 4 & 263 & 248\end{array}$

$\begin{array}{llll}2-12 & 4 & 240 & 202\end{array}$

3-12 $4 \quad 246 \quad 218$

$4-12 \quad 4 \quad 398 \quad 384$

$-10-11 \quad 4 \quad 390 \quad 407$

$-9-114753 \quad 783$

$-8-11 \quad 4 \quad 521 \quad 476$

$-7-11 \quad 4 \quad 406 \quad 409$

$-6-11 \quad 4 \quad 10651051$

$\begin{array}{llll}-5-11 & 4 & 338 & 328\end{array}$

$\begin{array}{llll}-4-11 & 4 & 1267 & 1226\end{array}$

$\begin{array}{lll}-3-11 & 4 & 234 \\ -225\end{array}$

$\begin{array}{llll}-2-11 & 4 & 733 & 757\end{array}$

$\begin{array}{llll}-1-11 & 4 & 276 & 261\end{array}$

$0-11 \quad 4 \quad 638 \quad 625$

$1-11 \quad 4 \quad 288 \quad 236$

$\begin{array}{llll}2-11 & 4 & 345 & 372\end{array}$

$3-11 \quad 4 \quad 208 \quad 207$

$\begin{array}{llll}-11-10 & 4 & 256 & 272\end{array}$

$-10-10 \quad 4 \quad 559 \quad 576$

$\begin{array}{llll}-9-10 & 4 & 722 & 711\end{array}$

$-8-10 \quad 4 \quad 780 \quad 815$

$\begin{array}{llll}-7-10 & 4 & 548 & 523\end{array}$

$-6-10 \quad 410801070$

$-4-10 \quad 4 \quad 1624 \quad 1594$ h $\mathrm{k}$ I 1OFO 1OEC

$-2-10 \quad 4 \quad 1128 \quad 1178$

$\begin{array}{llll}-1-10 & 4 & 507 & 532\end{array}$

0-10 4 662 682

$1-10 \quad 4 \quad 1140 \quad 1091$

$2-10 \quad 436 \quad 751$

$3-10 \quad 4 \quad 322 \quad 326$

4-10 $4 \quad 208 \quad 282$

$\begin{array}{lllll}-10 & -9 & 4 & 319 & 322\end{array}$

$\begin{array}{lllll}-9 & -9 & 4 & 413 & 385\end{array}$

$\begin{array}{lllll}-8 & -9 & 4 & 623 & 633\end{array}$

$\begin{array}{lllll}-7 & -9 & 4 & 454 & 468\end{array}$

$\begin{array}{lllll}-6 & -9 & 4 & 650 & 650\end{array}$

$\begin{array}{lllll}-5 & -9 & 4 & 236 & 239\end{array}$

$\begin{array}{lllll}-4 & -9 & 4 & 1346 & 1303\end{array}$

$\begin{array}{lllll}-3 & -9 & 4 & 291 & 321\end{array}$

$\begin{array}{llllll}-2 & -9 & 4 & 1267 & 1338\end{array}$

$\begin{array}{lllll}-1 & -9 & 4 & 352 & 384\end{array}$

$\begin{array}{lllll}0 & -9 & 4 & 184 & 125\end{array}$

$\begin{array}{lllll}1 & -9 & 4 & 1335 & 1343\end{array}$

$\begin{array}{lllll}3 & -9 & 4 & 1337 & 1356\end{array}$

$\begin{array}{lllll}4 & -9 \quad 4 & 372 \quad 353\end{array}$

$\begin{array}{lllll}5 & -9 & 4 & 427 & 441\end{array}$

$\begin{array}{lllll}7 & -9 & 4 & 318 & 312\end{array}$

$\begin{array}{lllll}-11 & -8 & 4 & 222 & 268\end{array}$

$\begin{array}{lllll}-9 & -8 & 4 & 257 & 215\end{array}$

$\begin{array}{lllll}-8 & -8 & 4 & 199 & 199\end{array}$

$\begin{array}{lllll}-7 & -8 & 4 & 443 & 431\end{array}$

$\begin{array}{lllll}-5 & -8 & 4 & 217 & 208\end{array}$

$\begin{array}{lllll}-4 & -8 & 4 & 256 & 282\end{array}$

$\begin{array}{lllll}-3 & -8 & 4 & 922 & 906\end{array}$

$\begin{array}{lllll}-2 & -8 & 4 & 1123 & 1132\end{array}$

$\begin{array}{lllll}-1 & -8 & 4 & 456 & 469\end{array}$

$\begin{array}{lllll}1 & -8 & 4 & 12121196\end{array}$

$\begin{array}{lllll}2 & -8 & 4 & 559 & 550\end{array}$ 


\begin{tabular}{|c|c|c|c|c|c|c|c|c|c|c|c|c|c|c|c|c|c|c|c|c|c|c|c|c|}
\hline 9 & 7 & & 54 & 4 & 12 & -5 & $\frac{4}{n}$ & 404 & 14 & $\sigma$ & -4 & & 082 & 815 & 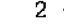 & -2 & $\frac{4}{6}$ & 2000 & 2004 & -6 & 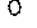 & & 13 & \\
\hline-8 & -7 & 4 & 518 & 521 & -10 & -5 & 4 & 957 & 944 & 7 & -4 & 4 & 198 & 196 & 3 & -2 & 4 & 738 & 759 & -5 & 0 & 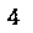 & 616 & 623 \\
\hline-7 & -7 & 4 & 502 & 483 & -9 & -5 & 4 & 587 & 611 & 8 & -4 & 4 & 408 & 397 & 4 & -2 & 4 & 2472 & 2486 & -4 & 0 & 4 & 2061 & 2034 \\
\hline-6 & -7 & 4 & 574 & 574 & -8 & -5 & 4 & 1143 & 1159 & 9 & -4 & 4 & 256 & 232 & 5 & -2 & 4 & 168 & 196 & -3 & 0 & 4 & 1560 & 1494 \\
\hline-5 & -7 & 4 & 483 & 511 & -7 & -5 & 4 & 649 & 634 & -10 & -3 & & 221 & 190 & 6 & -2 & 4 & 1240 & 1275 & -2 & 0 & 4 & 699 & 656 \\
\hline-4 & -7 & 4 & 752 & 722 & -5 & -5 & 4 & 2012 & 2039 & -9 & -3 & & 408 & 429 & 7 & -2 & 4 & 1030 & 1037 & -1 & 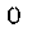 & 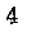 & 658 & 589 \\
\hline-3 & -7 & 4 & 411 & 441 & -3 & -5 & 4 & 2431 & 2392 & -8 & -3 & & 366 & 381 & 8 & -2 & 4 & 549 & 555 & 0 & 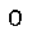 & 4 & 572 & 577 \\
\hline-2 & -7 & 4 & 161 & 166 & -2 & -5 & 4 & 204 & 214 & -7 & -3 & 4 & 902 & 929 & 9 & -2 & 4 & 933 & 922. & 1 & 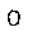 & 4 & 1577 & 1551 \\
\hline-1 & -7 & 4 & 553 & 537 & -1 & -5 & 4 & 676 & 710 & -6 & -3 & 4 & 292 & 320 & 10 & -2 & 4 & 257 & 224 & 2 & 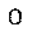 & 4 & 496 & 526 \\
\hline 0 & -7 & 4 & 371 & 378 & 0 & -5 & 4 & 444 & 449 & -5 & -3 & 4 & 1303 & 1298 & 11 & -2 & 4 & 524 & 510 & 3 & 0 & 4 & 1076 & 1090 \\
\hline 1 & -7 & 4 & 730 & 717 & 1 & -5 & 4 & 843 & 834 & -4 & -3 & 4 & 474 & 481 & -11 & -1 & 4 & 866 & 911 & 4 & 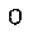 & 4 & 782 & 787 \\
\hline 2 & -7 & 4 & 551 & 545 & 2 & -5 & 4 & 895 & 883 & -3 & -3 & $\mathbf{4}$ & 2227 & 2208 & -9 & -1 & 4 & 902 & 915 & 5 & 0 & 4 & 868 & 899 \\
\hline 3 & -7 & 4 & 1470 & 1469 & 3 & -5 & 4 & 637 & 641 & -2 & -3 & 4 & 148 & 137 & -8 & -1 & 4 & 341 & 310 & 6 & 0 & 4 & 968 & 1031 \\
\hline 4 & -7 & 4 & 603 & 625 & 5 & -5 & 4 & 351 & 342 & -1 & -3 & 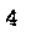 & 1476 & 1472 & -7 & -1 & 4 & 545 & 555 & 7 & 0 & 4 & 493 & 496 \\
\hline 5 & -7 & 4 & 971 & 1027 & 6 & -5 & 4 & 200 & 194 & 0 & -3 & 4 & 1622 & 1597 & -6 & -1 & 4 & 757 & 733 & 9 & 0 & 4 & 1025 & 1023 \\
\hline 6 & -7 & 4 & 570 & 561 & 7 & -5 & 4 & 224 & 228 & 1 & -3 & 4 & 260 & 228 & -5 & -1 & 4 & 791 & 776 & 11 & 0 & 4 & 662 & 656 \\
\hline 7 & -7 & 4 & 281 & 282 & 9 & -5 & 4 & 428 & 401 & 2 & -3 & 4 & 3197 & 3178 & -4 & -1 & 4 & 413 & 404 & -11 & 1 & 4 & 452 & 452 \\
\hline 8 & -7 & 4 & 796 & 815 & -12 & -4 & 4 & 232 & 100 & 3 & -3 & 4 & 504 & 517 & -3 & -1 & 4 & 710 & 670 & -10 & 1 & 4 & 401 & 393 \\
\hline-12 & -6 & 4 & 671 & 651 & -11 & -4 & 4 & 253 & 258 & 4 & -3 & 4 & 1976 & 1961 & -2 & -1 & 4 & 1200 & 1126 & -9 & 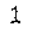 & 4 & 858 & 830 \\
\hline-10 & -6 & 4 & 1065 & 1072 & -10 & -4 & 4 & 315 & 391 & 5 & -3 & 4 & 212 & 229 & -1 & -1 & 4 & 1241 & 1216 & -8 & 1 & 4 & 380 & 394 \\
\hline-8 & -6 & 4 & 808 & 818 & -9 & -4 & 4 & 1001 & 974 & 6 & -3 & 4 & 620 & 619 & 0 & -1 & 4 & 294 & 322 & -7 & 1 & 4 & 961 & 966 \\
\hline-7 & -6 & 4 & 286 & 270 & -8 & -4 & 4 & 1063 & 1039 & 7 & -3 & 4 & 630 & 611 & 1 & -1 & 4 & 1147 & 1068 & -6 & 1 & 4 & 768 & 731 \\
\hline-5 & -6 & 4 & 1441 & 1445 & -7 & -4 & 4 & 1273 & 1297 & 8 & -3 & 4 & 437 & 437 & 2 & -1 & 4 & 372 & 336 & -5 & 1 & 4 & 230 & 253 \\
\hline-4 & -6 & 4 & 1168 & 1123 & -6 & -4 & 4 & 317 & 312 & 9 & -3 & 4 & 342 & 310 & 3 & -1 & 4 & 1222 & 1217 & -4 & L. & 4 & 2672 & 2641 \\
\hline-3 & -6 & 4 & 1050 & 1016 & -5 & -4 & 4 & 1864 & 1847 & 10 & -3 & 4 & 307 & 265 & 4 & -1 & 4 & 1907 & 1920 & -3 & 2 & 4 & 386 & 337 \\
\hline-2 & -6 & 4 & 966 & 940 & -4 & -4 & 4 & 335 & 348 & -11 & -2 & 4 & 615 & 625 & 5 & -1 & 4 & 1007 & 1025 & -2 & 1 & 4 & 2675 & 2572 \\
\hline
\end{tabular}

Observed and calculated structure factors: $\left.\quad \operatorname{lOS}(\mathrm{CO}) \mathrm{C}_{2} \mathrm{PPH}_{3}\right]_{2}(\mathrm{MU}-\mathrm{I})\left(\mathrm{M}-\mathrm{PPH}_{2}\right)$

Page 12

\begin{tabular}{|c|c|c|c|c|c|c|c|c|c|c|c|c|c|c|c|c|c|c|c|c|c|c|c|c|}
\hline $\mathrm{h}$ & $\mathrm{k}$ & 1 & 1OFO & $10 F C$ & $\mathrm{~h}$ & $\mathrm{k}$ & 1 & 10FO & $10 F C$ & h & $\mathrm{k}$ & $I$ & $10 \mathrm{FO}$ & $10 F C$ & $h$ & $\mathrm{k}$ & 1 & 10Fo & 10FC & $\mathrm{h}$ & $k$ & 1 & $10 \mathrm{FO}$ & $10 F \mathrm{C}$ \\
\hline 1 & 1 & 4 & 286 & 270 & -7 & 3 & 4 & 320 & 319 & 10 & 4 & 4 & 940 & 992 & 6 & 6 & 4 & 580 & 607 & 1 & 8 & 4 & 729 & 757 \\
\hline 0 & 1 & 4 & 2148 & 2141 & -6 & 3 & 4 & 737 & 764 & 11 & 4 & 4 & 225 & 194 & 7 & 6 & 4 & 648 & 671 & 2 & 8 & 4 & 1644 & 1633 \\
\hline 1 & 1 & 4 & 1493 & 1420 & -4 & 3 & 4 & 1175 & 1188 & 12 & 4 & 4 & 383 & 385 & 8 & 6 & 4 & 694 & 709 & 3 & 8 & 4 & 507 & 504 \\
\hline 2 & 1 & 4 & 2094 & 1979 & -3 & 3 & 4 & 1464 & 1431 & -8 & 5 & 4 & 769 & 763 & 10 & 6 & 4 & 903 & 940 & 4 & 8 & 4 & 1681 & 1684 \\
\hline 3 & 1 & 4 & 579 & 558 & -2 & 3 & 4 & 2290 & 2264 & -6 & 5 & 4 & 419 & 429 & 11 & 6 & 4 & 258 & 254 & 5 & 8 & 4 & 355 & 321 \\
\hline 4 & 1 & 4 & 655 & 649 & -1 & 3 & 4 & 1627 & 1554 & -5 & 5 & 4 & 796 & 787 & 12 & 6 & 4 & 541 & 576 & 6 & 8 & 4 & 878 & 852 \\
\hline 5 & 1 & 4 & 180 & 140 & 0 & 3 & 4 & 1622 & 1596 & -4 & 5 & 4 & 747 & 768 & -8 & 7 & 4 & 690 & 701 & 7 & $B$ & 4 & 764 & 774 \\
\hline 6 & 1 & 4 & 608 & 628 & 1 & 3 & 4 & 568 & 557 & -3 & 5 & 4 & 328 & 338 & -7 & 7 & 4 & 382 & 367 & 8 & $B$ & 4 & 918 & 921 \\
\hline 7 & 1 & 4 & 393 & 405 & 2 & 3 & 4 & 742 & 701 & 0 & 5 & 4 & 1033 & 997 & -6 & 7 & 4 & 450 & 439 & 10 & 8 & 4 & 288 & 293 \\
\hline 10 & 1 & 4 & 415 & 4.17 & 3 & 3 & 4 & 3481 & 3524 & 1 & 5 & 4 & 278 & 260 & -5 & 7 & 4 & 689 & 704 & -6 & 9 & 4 & 210 & 204 \\
\hline 11 & 1 & 4 & 494 & 498 & 4 & 3 & 4 & 211 & 190 & 2 & 5 & 4 & 766 & 725 & -3 & 7 & 4 & 2803 & 1800 & -4 & 9 & 4 & 460 & 451 \\
\hline 12 & 1 & 4 & 232 & 209 & 5 & 3 & 4 & 1633 & 1674 & 3 & 5 & 4 & 1142 & 1104 & -2 & 7 & 4 & 210 & 193 & -3 & 9 & 4 & 494 & 500 \\
\hline-10 & 2 & 4 & 311 & 352 & 6 & 3 & 4 & 308 & 274 & 4 & 5 & 4 & 1459 & 15.15 & -1 & 7 & 4 & 1364 & 1385 & -2 & 9 & 4 & 971 & 977 \\
\hline-9 & 2 & 4 & 295 & 274 & 7 & 3 & 4 & 816 & 831 & 5 & 5 & 4 & 1830 & 1822 & 0 & 7 & 4 & 433 & 394 & -1 & 9 & 4 & 1195 & 1221 \\
\hline-8 & 2 & 4 & 853 & 875 & 8 & 3 & 4 & 980 & 986 & 6 & 5 & 4 & 751 & 780 & 1 & 7 & 4 & 655 & 656 & 0 & 9 & 4 & 723 & 716 \\
\hline-7 & 2 & 4 & 664 & 695 & 9 & 3 & 4 & 981 & 994 & 7 & 5 & 4 & 688 & 636 & 2 & 7 & 4 & 1257 & 1260 & 1 & 9 & 4 & 402 & 435 \\
\hline-6 & 2 & 4 & 730 & 726 & 10 & 3 & 4 & 341 & 334 & 8 & 5 & 4 & 1107 & 1144 & 3 & 7 & 4 & 687 & 684 & 2 & 9 & 4 & 1059 & 1067 \\
\hline-5 & 2 & 4 & 411. & 416 & 11 & 3 & 4 & 396 & 390 & 9 & 5 & 4 & 266 & 281 & 4 & 7 & 4 & 507 & 510 & 4 & 9 & 4 & 1862 & 1872 \\
\hline-4 & 2 & 4 & 1973 & 1969 & -10 & 4 & 4 & 561 & 547 & 10 & 5 & 4 & 1239 & 1248 & 5 & 7 & 4 & 465 & 432 & 6 & 9 & 4 & 1187 & 1197 \\
\hline-3 & 2 & 4 & 825 & 819 & -9 & 4 & 4 & 300 & 316 & 12 & 5 & 4 & 697 & 722 & 7 & 7 & 4 & 455 & 470 & 7 & 9 & 4 & 896 & 913 \\
\hline-2 & 2 & 4 & 3192 & 3117 & -5 & 4 & 4 & 383 & 394 & -8 & 6 & 4 & 802 & 830 & 8 & 7 & 4 & 319 & 309 & 8 & 9 & 4 & 790 & 802 \\
\hline-1 & 2 & 4 & 1193 & 1113 & -3 & 4 & 4 & 535 & 515 & -7 & 6 & 4 & 237 & 249 & 9 & 7 & 4 & 335 & 336 & 9 & 9 & 4 & 691 & 687 \\
\hline 0 & 2 & 4 & 780 & 827 & -2 & 4 & 4 & 1063 & 1032 & -6 & 6 & 4 & 457 & 464 & 10 & 7 & 4 & 280 & 293 & 10 & 9 & 4 & 787 & 783 \\
\hline 1 & 2 & 4 & 2192 & 2149 & -1 & 4 & 4 & 933 & 895 & -5 & 6 & 4 & 923 & 912 & 11 & 7 & 4 & 275 & 289 & 11 & 9 & 4 & 208 & 1.71 \\
\hline 2 & 2 & 4 & 1558 & 1522 & 0 & 4 & 4 & 761 & 737 & -4 & 6 & 4 & 441 & 451 & 12 & 7 & 4 & 357 & 348 & 12 & 9 & 4 & 224 & 215 \\
\hline 3 & 2 & 4 & 2409 & 2434 & 1 & 4 & 4 & 9.12 & 926 & -3 & 6 & 4 & 1442 & 1435 & -8 & 8 & 4 & 315 & 317 & -2 & 10 & 4 & 488 & 456 \\
\hline 4 & 2 & 4 & 880 & 886 & 2 & 4 & 4 & 280 & 245 & -2 & 6 & 4 & 659 & 645 & -7 & 8 & 4 & 286 & 313 & -1 & 10 & 4 & 541 & 535 \\
\hline 5 & 2 & 4 & 881 & 905 & 3 & 4 & 4 & 2655 & 2677 & -1 & 6 & 4 & 699 & 639 & -6 & 8 & 4 & 332 & 324 & 0 & 10 & 4 & 736 & 719 \\
\hline 7 & 2 & 4 & 838 & 889 & 4 & 4 & 4 & 827 & 846 & 0 & 6 & 4 & 169 & 189 & -5 & 8 & 4 & 353 & 377 & 1 & 10 & 4 & 460 & 445 \\
\hline 9 & 2 & 4 & 621 & 649 & 5 & 4 & 4 & 1999 & 2000 & 1 & 6 & 4 & 719 & 703 & -4 & 8 & 4 & 636 & 632 & 2 & 10 & 4 & 564 & 557 \\
\hline 10 & 2 & 4 & 403 & 409 & 6 & 4 & 4 & 319 & 325 & 2 & 6 & 4 & 272 & 280 & -3 & 8 & 4 & 1315 & 1348 & 3 & 10 & 4 & 601 & 575 \\
\hline 12 & 2 & 4 & 271 & 224 & 7 & 4 & 4 & 647 & 675 & 3 & 6 & 4 & 975 & 945 & -2 & 8 & 4 & 642 & 635 & 4 & 10 & 4 & 1502 & 1510 \\
\hline-9 & 3 & 4 & 283 & 252 & 8 & 4 & 4 & 1356 & 1371 & 4 & 6 & 4 & 689 & 725 & -1 & 8 & 4 & 1663 & 1661 & 5 & 10 & 4 & 424 & 436 \\
\hline 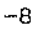 & 3 & 4 & 573 & 556 & 9 & 4 & 4 & 451 & 432 & 5 & 6 & 4 & 532 & 558 & 0 & 8 & 4 & 372 & 351 & 6 & 10 & 4 & 866 & 883 \\
\hline
\end{tabular}


$211 \$ 397 \quad 404$

$\begin{array}{lllll}3 & 11 & 4 & 449 & 455\end{array}$

$\begin{array}{lllll}4 & 11 & 4 & 388 & 367\end{array}$

$\begin{array}{lllll}5 & 11 & 4 & 605 & 625\end{array}$

$6114676 \quad 705$

$711 \quad 4 \quad 645 \quad 630$

$9114849 \quad 809$

$\begin{array}{lllll}11 & 11 & 4 & 865 & 866\end{array}$

$\begin{array}{lllll}-4 & 12 \quad 4 & 644 & 617\end{array}$

$\begin{array}{lllll}-2 & 12 & 4 & 671 & 661\end{array}$

$\begin{array}{lllll}0 & 12 & 4 & 284 & 296\end{array}$

$\begin{array}{lllll}1 & 12 & 4 & 491 & 491\end{array}$

$212 \quad 4 \quad 405 \quad 444$

$\begin{array}{lllll}4 & 12 \quad 4 & 353 & 359\end{array}$

$5124454 \quad 461$

$\begin{array}{lllll}6 & 12 \quad 4 \quad 230 & 200\end{array}$

$\begin{array}{lllll}7 & 12 & 4 & 355 & 354\end{array}$

$912 \quad 4 \quad 400 \quad 382$

$\begin{array}{lllll}10 & 12 & 4 & 291 & 251\end{array}$

$\begin{array}{lllll}11 & 12 & 4 & 487 & 511\end{array}$

$\begin{array}{lllll}-2 & 13 & 4 & 883 & 854\end{array}$

- $134515 \quad 518$

$113 \leadsto 430431$

$2134368 \quad 405$

$3134779 \quad 729$

$413 \quad 4 \quad 512 \quad 538$ $\begin{array}{lllll}8 & 14 & 4 & 322 & 287\end{array}$

$914 \quad 4 \quad 302 \quad 362$

$1014 \quad 4 \quad 257 \quad 222$

$\begin{array}{lllll}0 & 15 & 4 & 330 & 356\end{array}$

$\begin{array}{lllll}1 & 15 & 4 & 441 & 404\end{array}$

$\begin{array}{lllll}3 & 15 & 4 & 627 & 636\end{array}$

$\begin{array}{lllll}5 & 15 & 4 & 716 & 697\end{array}$

$\begin{array}{lllll}6 & 15 & 4 & 264 & 239\end{array}$

$\begin{array}{lllll}7 & 15 & 4 & 493 & 491\end{array}$

$\begin{array}{lllll}8 & 15 & 4 & 455 & 461\end{array}$

$\begin{array}{lllll}3 & 16 & 4 & 362 & 345\end{array}$

$\begin{array}{lllll}4 & 16 & 4 & 227 & 179\end{array}$

$\begin{array}{lllll}5 & 16 & 4 & 555 & 547\end{array}$

$616 \quad 4 \quad 289 \quad 293$

$\begin{array}{llll}-6-14 & 5 & 241 & 222\end{array}$

$\begin{array}{llll}-5-14 & 5 & 31.9 & 306\end{array}$

$\begin{array}{llll}-4-14 & 5 & 433 & 428\end{array}$

$\begin{array}{llll}-3-14 & 5 & 590 & 647\end{array}$

$\begin{array}{llll}-2-14 & 5 & 533 & 583\end{array}$

$\begin{array}{llll}-1-14 & 5 & 457 & 483\end{array}$

$\begin{array}{llll}-3-13 & 5 & 674 & 687\end{array}$

$\begin{array}{llll}-2-13 & 5 & 288 & 255\end{array}$

$\begin{array}{llll}-1-13 & 5 & 688 & 674\end{array}$

1-13 $5 \quad 564 \quad 536$

$\begin{array}{llll}2-13 & 5 & 259 & 247\end{array}$

$\begin{array}{llll}-8-12 & 5 & 384 & 373\end{array}$
$-9-11 \quad 5 \quad 503 \quad 509$

$\begin{array}{llll}-8-11 & 5 & 741 . & 749\end{array}$

$\begin{array}{llll}-7-11 & 5 & 475 & 441\end{array}$

$\begin{array}{llll}-6-11 & 5 & 2.56 & 203\end{array}$

$\begin{array}{llll}-5-11 & 5 & 1052 & 1032\end{array}$

$\begin{array}{llll}-3-11 & 5 & 665 & 721\end{array}$

$\begin{array}{llll}-2-11 & 5 & 315 & 343\end{array}$

$\begin{array}{llll}-1-11 & 5 & 202 & 135\end{array}$

0-11 $5 \begin{array}{lll}581 & 376\end{array}$

$\begin{array}{llll}-10-10 & 5 & 423 & 451\end{array}$

$\begin{array}{llll}-9-10 & 5 & 947 & 990\end{array}$

$\begin{array}{llll}-8-10 & 5 & 713 & 690\end{array}$

$\begin{array}{llll}-7-10 & 5 & 842 & 817\end{array}$

$\begin{array}{llll}-6-10 & 5 & 321 & 305\end{array}$

$\begin{array}{llll}-5-10 & 5 & 1240 & 1237\end{array}$

$\begin{array}{llll}-4-10 & 5 & 229 & 252\end{array}$

$\begin{array}{llll}-3-10 & 5 & 1372 & 1424\end{array}$

$\begin{array}{llll}-1-10 & 5 & 826 & 841\end{array}$

$0-10 \quad 5 \quad 730 \quad 720$

$1-10 \quad 5 \quad 348 \quad 361$

2-10 $5 \quad 595 \quad 578$

$\begin{array}{llll}3-10 & 5 & 326 & 327\end{array}$

$\begin{array}{lllll}-9 & -9 & 5 & 864 & 898\end{array}$

$\begin{array}{lllll}-8 & -9 & 5 & 217 & 237\end{array}$

$\begin{array}{lllll}-7 & -9 & 5 & 1220 & 1228\end{array}$

$\begin{array}{lllll}-6 & -9 & 5 & 272 & 247\end{array}$ $\begin{array}{lllll}4 & -9 & 5 & 541 & 551\end{array}$

$\begin{array}{lllll}-9 & -8 & 5 & 337 & 302\end{array}$

$\begin{array}{lllll}-7 & -8 & 5 & 1041 & 1042\end{array}$

$\begin{array}{lllll}-6 & -8 & 5 & 270 & 297\end{array}$

$\begin{array}{lllll}-5 & -8 & 5 & 426 & 402\end{array}$

$\begin{array}{lllll}-4 & -8 & 5 & 748 & 741\end{array}$

$\begin{array}{lllll}-3 & -8 & 5 & 892 & 888\end{array}$

$\begin{array}{lllll}-2 & -8 & 5 & 874 & 877\end{array}$

$\begin{array}{lllll}-1 & -8 & 5 & 901 & 900\end{array}$

$\begin{array}{lllll}0 & -8 & 5 & 1031 & 1034\end{array}$

$\begin{array}{lllll}1 & -8 & 5 & 295 & 276\end{array}$

$\begin{array}{lllll}2 & -8 & 5 & 1495 & 1478\end{array}$

$\begin{array}{lllll}3 & -8 & 5 & 557 & 537\end{array}$

$\begin{array}{lllll}4 & -8 & 5 & 1353 & 1377\end{array}$

$\begin{array}{lllll}6 & -8 & 5 & 398 & 41.2\end{array}$

$\begin{array}{lllll}7 & -8 & 5 & 455 & 419\end{array}$

$\begin{array}{lllll}-11 & -7 & 5 & 630 & 581\end{array}$

$\begin{array}{lllll}-9 & -7 & 5 & 580 & 567\end{array}$

$\begin{array}{lllll}-8 & -7 & 5 & 214 & 231\end{array}$

$\begin{array}{lllll}-6 & -7 & 5 & 377 & 395\end{array}$

$\begin{array}{lllll}-5 & -7 & 5 & 255 & 175\end{array}$

$\begin{array}{lllll}-4 & -7 & 5 & 173 & 177\end{array}$

$\begin{array}{lllll}-2 & -7 & 5 & 1100 & 1158\end{array}$

$\begin{array}{lllll}-1 & -7 & 5 & 533 & 511\end{array}$

$\begin{array}{lllll}0 & -7 & 5 & 1038 & 1041\end{array}$

$\begin{array}{llllll}2 & -7 & 5 & 1179 & 1176\end{array}$ $\begin{array}{lllll}-5 & -6 & 5 & 858 & 842 \\ -5 & 254 & 278\end{array}$

$\begin{array}{lllll}-4 & -6 & 5 & 815 & 822\end{array}$

$\begin{array}{lllll}-3 & -6 & 5 & 1248 & 1223\end{array}$

$\begin{array}{lllll}-2 & -6 & 5 & 502 & 499\end{array}$

$\begin{array}{lllll}-1 & -6 & 5 & 275 & 271\end{array}$

$\begin{array}{lllll}0 & -6 & 5 & 1126 & 1165\end{array}$

$\begin{array}{lllll}2 & -6 & 5 & 617 & 580\end{array}$

$\begin{array}{lllll}3 & -6 & 5 & 711 & 714\end{array}$

$\begin{array}{lllll}4 & -6 & 5 & 786 & 784\end{array}$

$\begin{array}{lllll}5 & -6 & 5 & 859 & 878\end{array}$

$\begin{array}{lllll}6 & -6 & 5 & 586 & 581\end{array}$

$\begin{array}{lllll}7 & -6 & 5 & 585 & 584\end{array}$

$\begin{array}{lllll}9 & -6 & 5 & 830 & 819\end{array}$

$\begin{array}{lllll}-11 & -5 & 5 & 560 & 579\end{array}$

$\begin{array}{lllll}-10 & -5 & 5 & 532 & 501\end{array}$

$\begin{array}{lllll}-9 & -5 & 5 & 1065 & 1058\end{array}$

$\begin{array}{lllll}-8 & -5 & 5 & 400 & 398\end{array}$

$\begin{array}{lllll}-7 & -5 & 5 & 801 & 796\end{array}$

$\begin{array}{lllll}-6 & -5 & 5 & 940 & 939\end{array}$

$\begin{array}{lllll}-4 & -5 & 5 & 1916 & 1932\end{array}$

$\begin{array}{lllll}-2 & -5 & 5 & 1020 & 1004\end{array}$

$\begin{array}{lllll}-1 & -5 & 5 & 416 & 374\end{array}$

$\begin{array}{lllll}0 & -5 & 5 & 513 & 487\end{array}$

$\begin{array}{lllll}1 & -5 & 5 & 327 & 339\end{array}$

$\begin{array}{lllll}2 & -5 & 5 & 243 & 239\end{array}$

Observed and calculated structure factors: $\quad\left[\mathrm{OS}(\mathrm{CO}){ }_{2} \mathrm{PPH}_{3}\right]_{2}$ (MU-I) (MU-PPH ${ }_{2}$ )

Page 14

h k 1 10FO 10FC

$\begin{array}{lllll}3 & -5 & 5 & 267 & 262\end{array}$

$\begin{array}{lllll}5 & -5 & 5 & 318 & 333\end{array}$

$\begin{array}{lllll}6 & -5 & 5 & 290 & 310\end{array}$

$\begin{array}{lllll}7 & -5 & 5 & 262 & 254\end{array}$

$\begin{array}{lllll}8 & -5 & 5 & 407 & 394\end{array}$

$\begin{array}{lllll}9 & -5 & 5 & 532 & 529\end{array}$

$\begin{array}{lllll}-10 & -4 & 5 & 664 & 637\end{array}$

$\begin{array}{lllll}-9 & -4 & 5 & 628 & 652\end{array}$

$\begin{array}{lllll}-8 & -4 & 5 & 1326 & 1317\end{array}$

$\begin{array}{lllll}-7 & -4 & 5 & 700 & 685\end{array}$

$\begin{array}{lllll}-6 & -4 & 5 & 1252 & 1257\end{array}$

$\begin{array}{lllll}-5 & -4 & 5 & 475 & 448\end{array}$

$\begin{array}{lllll}-4 & -4 & 5 & 2236 & 2235\end{array}$

$\begin{array}{lllll}-3 & -4 & 5 & 892 & 836\end{array}$

$\begin{array}{lllll}-2 & -4 & 5 & 2467 & 2443\end{array}$

$\begin{array}{lllll}-1 & -4 & 5 & 744 & 745\end{array}$

$\begin{array}{lllll}0 & -4 & 5 & 508 & 492\end{array}$

$\begin{array}{lllll}1 & -4 & 5 & 976 & 996\end{array}$

$\begin{array}{lllll}2 & -4 & 5 & 500 & 532\end{array}$

$\begin{array}{lllll}3 & -4 & 5 & 899 & 914\end{array}$

$\begin{array}{lllll}4 & -4 & 5 & 449 & 482\end{array}$

$\begin{array}{lllll}6 & -4 & 5 & 227 & 220\end{array}$

$\begin{array}{lllll}10 & -4 & 5 & 477 & 483\end{array}$

$\begin{array}{lllll}-10 & -3 & 5 & 338 & 298\end{array}$

$\begin{array}{llllll}-8 & -3 & 5 & 1347 & 1400\end{array}$

$\begin{array}{lllll}-7 & -3 & 5 & 352 & 335\end{array}$

$\begin{array}{lllll}-6 & -3 & 5 & 1255 & 1249\end{array}$

$\begin{array}{lllll}-4 & -3 & 5 & 1650 & 1612\end{array}$

$\begin{array}{lllll}-3 & -3 & 5 & 1568 & 1524\end{array}$

$\begin{array}{lllll}-2 & -3 & 5 & 2968 & 2942\end{array}$

$\begin{array}{lllll}-1 & -3 & 5 & 1619 & 1629\end{array}$

$\begin{array}{llllll}0 & -3 & 5 & 1472 & 1484\end{array}$

$\begin{array}{llllll}1 & -3 & 5 & 1913 & 1871\end{array}$

$\begin{array}{lllll}2 & -3 & 5 & 154 & 48\end{array}$ h $k 1$ 1OFO 1OFC

$\begin{array}{lllll}3 & -3 & 5 & 2517 & 2482\end{array}$

$\begin{array}{lllll}4 & -3 & 5 & 330 & 346\end{array}$

$\begin{array}{lllll}5 & -3 & 5 & 846 & 836\end{array}$

$\begin{array}{lllll}6 & -3 & 5 & 279 & 327\end{array}$

$\begin{array}{lllll}7 & -3 & 5 & 178 & 150\end{array}$

$\begin{array}{lllll}8 & -3 & 5 & 558 & 555\end{array}$

$\begin{array}{lllll}9 & -3 & 5 & 410 & 401\end{array}$

$\begin{array}{lllll}-10 & -2 & 5 & 308 & 323\end{array}$

$\begin{array}{lllll}-9 & -2 & 5 & 249 & 276\end{array}$

$\begin{array}{lllll}-8 & -2 & 5 & 557 & 560\end{array}$

$\begin{array}{llllll}-6 & -2 & 5 & 778 & 805\end{array}$

$\begin{array}{lllll}-5 & -2 & 5 & 265 & 257\end{array}$

$\begin{array}{lllll}-4 & -2 & 5 & 461 & 468\end{array}$

$\begin{array}{lllll}-3 & -2 & 5 & 1170 & 1149\end{array}$

$\begin{array}{lllll}-2 & -2 & 5 & 1692 & 1630\end{array}$

$\begin{array}{llllll}-1 & -2 & 5 & 2197 & 2172\end{array}$

o $\quad-2 \quad 5 \quad 1537 \quad 1550$

$\begin{array}{lllll}1 & -2 & 5 & 1662 & 1603\end{array}$

$\begin{array}{lllll}2 & -2 & 5 & 1149 & 1116\end{array}$

$\begin{array}{lllll}3 & -2 & 5 & 2077 & 2044\end{array}$

$\begin{array}{lllll}4 & -2 & 5 & 566 & 552\end{array}$

$\begin{array}{lllll}5 & -2 & 5 & 1778 & 1845\end{array}$

$\begin{array}{lllll}6 & -2 & 5 & 188 & 181\end{array}$

$\begin{array}{lllll}7 & -2 & 5 & 317 & 333\end{array}$

$\begin{array}{lllll}8 & -2 & 5 & 1048 & 1044\end{array}$

$\begin{array}{lllll}9 & -2 & 5 & 237 & 255\end{array}$

$\begin{array}{lllll}10 & -2 & 5 & 630 & 585\end{array}$

$\begin{array}{lllll}-10 & -1 & 5 & 682 & 750\end{array}$

$\begin{array}{lllll}-8 & -1 & 5 & 499 & 509\end{array}$

$\begin{array}{lllll}-6 & -1 & 5 & 185 & 194\end{array}$

$\begin{array}{lllll}-5 & -1 & 5 & 766 & 751\end{array}$

$\begin{array}{lllll}-4 & -1 & 5 & 336 & 336\end{array}$

$\begin{array}{lllll}-3 & -1 & 5 & 710 & 665\end{array}$

$\begin{array}{lllll}-2 & -1 & 5 & 182 & 127\end{array}$ h $\mathrm{k}$ I 10FO 10FC

$\begin{array}{lllll}-1 & -1 & 5 & 1614 & 1539\end{array}$

$\begin{array}{lllll}0 & -1 & 5 & 637 & 644\end{array}$

$\begin{array}{lllll}1 & -1 & 5 & 865 & 819\end{array}$

$\begin{array}{lllll}2 & -1 & 5 & 1762 & 1766\end{array}$

$\begin{array}{lllll}3 & -1 & 5 & 1467 & 1483\end{array}$

$\begin{array}{lllll}4 & -1 & 5 & 1730 & 1710\end{array}$

$\begin{array}{lllll}5 & -1 & 5 & 1787 & 1846\end{array}$

$\begin{array}{lllll}6 & -1 & 5 & 959 & 955\end{array}$

$\begin{array}{lllll}7 & -1 & 5 & 530 & 538\end{array}$

$\begin{array}{lllll}8 & -1 & 5 & 1285 & 1296\end{array}$

$\begin{array}{llllll}10 & -1 & 5 & 1056 & 10.34\end{array}$

$\begin{array}{rllll}-10 & 0 & 5 & 865 & 872\end{array}$

$\begin{array}{lllll}-8 & 0 & 5 & 1081 & 1082\end{array}$

$\begin{array}{lllll}-7 & 0 & 5 & 200 & 160\end{array}$

$\begin{array}{lllll}-6 & 0 & 5 & 488 & 457\end{array}$

$\begin{array}{lllll}-5 & 0 & 5 & 1052 & 1082\end{array}$

$\begin{array}{lllll}-4 & 0 & 5 & 799 & 763\end{array}$

$\begin{array}{lllll}-3 & 0 & 5 & 909 & 909\end{array}$

$\begin{array}{lllll}-2 & 0 & 5 & 1614 & 1571\end{array}$

$\begin{array}{lllll}-1 & 0 & 5 & 647 & 635\end{array}$

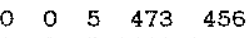

$100 \quad 5 \quad 1441 \quad 1361$

$\begin{array}{lllll}2 & 0 & 5 & 701 & 652\end{array}$

$\begin{array}{llll}0 & 5 & 1669 & 1671\end{array}$

$\begin{array}{lllll}5 & 0 & 5 & 1247 & 1225\end{array}$

$\begin{array}{llllll}6 & 0 & 5 & 1350 & 1398\end{array}$

$\begin{array}{lllll}7 & 0 & 5 & 565 & 561\end{array}$

$\begin{array}{lllll}8 & 0 & 5 & 837 & 854\end{array}$

$\begin{array}{lllll}9 & 0 & 5 & 321 & 330\end{array}$

$\begin{array}{lllll}10 & 0 & 5 & 904 & 949\end{array}$

$\begin{array}{lllll}11 & 0 & 5 & 457 & 460\end{array}$

$\begin{array}{lllll}-10 & 1 & 5 & 700 & 685\end{array}$

$\begin{array}{rrrrr}-9 & 1 & 5 & 559 & 579 \\ -8 & 1 & 5 & 978 & 1009\end{array}$ h $\mathrm{k} l 10 \mathrm{FO}$ 1OFC

$\begin{array}{lllll}-7 & 1 & 5 & 403 & 388\end{array}$

$\begin{array}{lllll}-6 & 1 & 5 & 756 & 791\end{array}$

$\begin{array}{lllll}-5 & 1 & 5 & 1317 & 1308\end{array}$

$\begin{array}{lllll}-4 & 1 & 5 & 332 & 299\end{array}$

$\begin{array}{lllll}-3 & 1 & 5 & 2359 & 2300\end{array}$

$\begin{array}{llllr}-2 & 1 & 5 & 378 & 359\end{array}$

$\begin{array}{lllll}-1 & 1 & 5 & 783 & 736\end{array}$

$0 \quad 151137 \quad 1113$

$\begin{array}{lllll}1 & 1 & 5 & 1597 & 1550\end{array}$

$2 \quad 1 \quad 5 \quad 1157 \quad 1093$

$\begin{array}{lllll}3 & 1 & 5 & 440 & 445\end{array}$

$\begin{array}{lllll}4 & 1 & 5 & 291 & 251\end{array}$

$\begin{array}{lllll}5 & 1 & 5 & 477 & 461\end{array}$

$\begin{array}{lllll}6 & 1 & 5 & 404 & 417\end{array}$

$\begin{array}{lllll}7 & 1 & 5 & 263 & 235\end{array}$

$\begin{array}{lllll}8 & 1 & 5 & 369 & 356\end{array}$

$\begin{array}{lllll}9 & 1 & 5 & 409 & 411\end{array}$

$\begin{array}{lllll}10 & 1 & 5 & 656 & 648\end{array}$

$\begin{array}{lllll}11 & 1 & 5 & 584 & 594\end{array}$

$\begin{array}{lllll}12 & 1 & 5 & 446 & 430\end{array}$

$\begin{array}{lllll}-9 & 2 & 5 & 753 & 760\end{array}$

$\begin{array}{lllll}-8 & 2 & 5 & 401 & 381\end{array}$

$\begin{array}{lllll}-7 & 2 & 5 & 855 & 845\end{array}$

$\begin{array}{lllll}-6 & 2 & 5 & 611 & 601\end{array}$

$\begin{array}{lllll}-5 & 2 & 5 & 1139 & 3115\end{array}$

$\begin{array}{lllll}-4 & 2 & 5 & 1000 \quad 964\end{array}$

$\begin{array}{lllll}-3 & 2 & 5 & 2657 & 2634\end{array}$

$\begin{array}{lllll}-2 & 2 & 5 & 1066 & 1029\end{array}$

$\begin{array}{lllll}-1 & 2 & 5 & 2172 & 2078\end{array}$

$\begin{array}{lllll}0 & 2 & 5 & 178 & 147\end{array}$

$\begin{array}{lllll}1 & 2 & 5 & 141 & 142\end{array}$

$\begin{array}{lllll}2 & 2 & 5 & 2182 & 2150\end{array}$

$\begin{array}{lllll}4 & 2 & 5 & 1310 & 1314\end{array}$

$\begin{array}{lllll}5 & 2 & 5 & 158 & 147\end{array}$

h k 1 1OFO 1OFC

$\begin{array}{lllll}6 & 2 & 5 & 324 & 322\end{array}$

$\begin{array}{lllll}7 & 2 & 5 & 283 & 272\end{array}$

$\begin{array}{lllll}8 & 2 & 5 & 254 & 267\end{array}$

$\begin{array}{lllll}11 & 2 & 5 & 581 & 534\end{array}$

$\begin{array}{lllll}12 & 2 & 5 & 281 & 261\end{array}$

$\begin{array}{lllll}-9 & 3 & 5 & 430 & 433\end{array}$

$\begin{array}{lllll}-7 & 3 & 5 & 983 & 1016\end{array}$

$\begin{array}{lllll}-6 & 3 & 5 & 227 & 167\end{array}$

$\begin{array}{lllll}-5 & 3 & 5 & 1196 & 1180\end{array}$ 


\begin{tabular}{|c|c|c|c|c|c|c|c|c|c|c|c|c|c|c|c|c|c|c|c|c|c|c|c|}
\hline-9 & 5 & 5 & 732 & 730 & 8 & 6 & 5 & 242 & 197 & 8 & 8 & 5 & 447 & 435 & 12 & 10 & 5 & 90 & 361 & 14 & 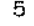 & 51 & 160 \\
\hline-7 & 5 & 5 & 374 & 380 & 9 & 6 & 5 & 714 & 706 & 9 & 8 & 5 & 227 & 250 & -3 & 11 & 5 & 212 & 245 & 015 & 5 & 435 & 438 \\
\hline-4 & 5 & 5 & 413 & 448 & 10 & 6 & 5 & 688 & 697 & 12 & 8 & 5 & 397 & 366 & -1 & 11 & 5 & 230 & 219 & 115 & 5 & 390 & 353 \\
\hline-3 & 5 & 5 & 381 & 358 & 11 & 6 & 5 & 940 & 928 & -6 & 9 & 5 & 451 & 460 & 1. & 11 & 5 & 458 & 4.58 & 215 & 5 & 329 & 332 \\
\hline-2 & 5 & 5 & 538 & 504 & 12 & 6 & 5 & 549 & 579 & -4 & 9 & 5 & 392 & 411 & 3 & 11 & 5 & 378 & 340 & 415 & 5 & 609 & 609 \\
\hline-1 & 5 & 5 & 680 & 627 & -8 & 7 & 5 & 444 & 452 & -3 & 9 & 5 & 877 & 872 & 4 & 11 & 5 & 870 & 826 & 615 & 5 & 522 & 552 \\
\hline 0 & 5 & 5 & 825 & 839 & -7 & 7 & 5 & 772 & 740 & -2 & 9 & 5 & 984 & 972 & 5 & 11 & 5 & 888 & 895 & 715 & 5 & 312 & 277 \\
\hline 1 & 5 & 5 & 630 & 639 & -6 & 7 & 5 & 456 & 454 & -1 & 9 & 5 & 1298 & 1270 & 6 & 11 & 5 & 807 & 788 & 815 & 5 & 355 & 359 \\
\hline 2 & 5 & 5 & 764 & 741 & -4 & 7 & 5 & 1130 & 1136 & 0 & 9 & 5 & 1134 & 1163 & 7 & 11 & 5 & 512 & 503 & 21.6 & & 263 & 305 \\
\hline 3 & 5 & 5 & 959 & 968 & -2 & 7 & 5 & 1622 & 1581 & 1 & 9 & 5 & 822 & 8.14 & 8 & 11 & 5 & 646 & 645 & 416 & 5 & 476 & 466 \\
\hline 4 & 5 & 5 & 1861 & 1867 & -1 & 7 & 5 & 233 & 253 & 3 & 9 & 5 & 1251 & 1229 & 10 & 11 & 5 & 896 & 885 & 516 & 5 & 469 & 461 \\
\hline 5 & 5 & 5 & 1288 & 1360 & 0 & 7 & 5 & 719 & 717 & 4 & 9 & 5 & 189 & 208 & -3 & 12 & 5 & 528 & 540 & 616 & 5 & 39 & 471 \\
\hline 6 & 5 & 5 & 1397 & 1446 & 1 & 7 & 5 & 496 & 482 & 5 & 9 & 5 & 1644 & 1620 & -1 & 12 & 5 & 391 & 382 & $-5-14$ & 5 & 416 & 416 \\
\hline 7 & 5 & 5 & 846 & 849 & 3 & 7 & 5 & 334 & 321 & 6 & 9 & 5 & 408 & 409 & 2 & 12 & 5 & 194 & 218 & $-4-14$ & 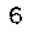 & 513 & 521 \\
\hline 8 & 5 & 5 & 227 & 132 & 4 & 7 & 5 & 760 & 743 & 7 & 9 & 5 & 556 & 562 & 4 & 12 & 5 & 248 & 271 & $-3-14$ & 5 & 547 & 544 \\
\hline 9 & 5 & 5 & 1242 & 1229 & 5 & 7 & 5 & 459 & 485 & 8 & 9 & 5 & 592 & 584 & 6 & 12 & 5 & 834 & 813 & $-2-14$ & 6 & 627 & 651 \\
\hline 10 & 5 & 5 & 323 & 326 & 6 & 7 & 5 & 211 & 222 & 9 & 9 & 5 & 337 & 339 & 7 & 12 & 5 & 342 & 331 & $-1-14$ & 6 & 443 & 437 \\
\hline 11 & 5 & 5 & 858 & 883 & 7 & 7 & 5 & 706 & 699 & 10 & 9 & 5 & 387 & 384 & 8 & 12 & 5 & 498 & 518 & $0-14$ & 6 & 354 & 394 \\
\hline 12 & 5 & 5 & 265 & 216 & 8 & 7 & 5 & 277 & 293 & 11 & 9 & 5 & 310 & 291 & 9 & 12 & 5 & 210 & 206 & $-7-13$ & 6 & 336 & 351 \\
\hline-9 & 6 & 5 & 687 & 701 & 9 & 7 & 5 & 363 & 345 & -6 & 10 & 5 & 294 & 266 & 10 & 12 & 5 & 573 & 571 & $-4-13$ & 6 & 473 & 475 \\
\hline-8 & 6 & 5 & 317 & 318 & 10 & 7 & 5 & 495 & 497 & -4 & 10 & 5 & 210 & 204 & 11 & 12 & 5 & 509 & 489 & $-2-13$ & 6 & 964 & 969 \\
\hline-7 & 6 & 5 & 788 & 782 & 11 & 7 & 5 & 609 & 579 & -3 & 10 & 5 & 219 & 216 & -3 & 13 & 5 & 585 & 566 & $-1-13$ & 6 & 296 & 273 \\
\hline-6 & 6 & 5 & 317 & 333 & 12 & 7 & 5 & 558 & 546 & -1 & 10 & 5 & 1042 & 1030 & -1 & 13 & 5 & 716 & 702 & $0-13$ & 6 & 742 & 705 \\
\hline-4 & 6 & 5 & 1024 & 1060 & -7 & 8 & 5 & 362 & 35.1 & 0 & 10 & 5 & 579 & 561 & 1 & 13 & 0 & 341 & 376 & $2-13$ & 6 & 426 & 428 \\
\hline-3 & 6 & 5 & 272 & 281 & -6 & 8 & 5 & 546 & 527 & 1 & 10 & 5 & 1045 & 1086 & 2 & 13 & 5 & 316 & 347 & $-9-12$ & 6 & 354 & 363 \\
\hline-2 & 6 & 5 & 512 & 537 & -4 & 8 & 5 & 768 & 757 & 3 & 10 & 5 & 1092 & 1096 & 3 & 13 & 5 & 232 & 213 & $-3-12$ & 6 & 311 & 273 \\
\hline
\end{tabular}

Observed and calculated structure factors: $\quad\left[O S(C O)_{2} \mathrm{PPH}_{3}\right]_{2}$ (MU-I) (MU-PPH ${ }_{2}$ )

Page 16

\begin{tabular}{|c|c|c|c|c|c|c|c|c|c|c|c|c|c|c|c|c|c|c|c|c|c|c|c|}
\hline $\mathrm{h} k$ & I & DEO & FC & $\mathrm{h}$ & $\mathrm{k}$ & & FO & $10 \mathrm{Fc}$ & h & k & 1 & IOFO & $10 \mathrm{E}$ & h & $\mathrm{k}$ & & $10 F O$ & 10 & h & $\mathrm{k}$ & 1 & 10FO & \\
\hline $2-12$ & 6 & 619 & 614 & -1 & -9 & 6 & 539 & 515 & -5 & -6 & 6 & 326 & 322 & -5 & -4 & 6 & 1027 & 1038 & 0 & -2 & 6 & 2211 & \\
\hline $0-12$ & 6 & 737 & 766 & 0 & -9 & 6 & 513 & 521 & -4 & -6 & 6 & 547 & 567 & -4 & -4 & 6 & 698 & 683 & 1 & -2 & 6 & 423 & \\
\hline $2-12$ & 6 & 420 & 411 & 1 & -9 & 6 & 722 & 718 & -2 & -6 & 6 & 502 & 505 & -3 & -4 & 6 & 1941 & 1935 & 2 & -2 & 6 & 1695 & 17 \\
\hline $3-12$ & 6 & 592 & 578 & 3 & -9 & 6 & 810 & 814 & -1 & -6 & 6 & 1838 & 1896 & -2 & -4 & 6 & 541 & 541. & 3 & -2 & 6 & 708 & \\
\hline $10-11$ & 6 & 354 & 357 & 4 & -9 & 6 & 279 & 283 & 1 & -6 & 6 & 1256 & 1267 & -1 & -4 & & 914 & 948 & 4 & -2 & 6 & 2099 & \\
\hline$-9-11$ & 6 & 727 & 717 & -10 & -8 & 6 & 221 & 234 & 2 & -6 & 6 & 580 & 607 & 0 & -4 & 6 & 147 & 74 & 5 & -2 & 6 & 180 & \\
\hline$-8-11$ & 6 & 349 & 350 & -8 & -8 & 6 & 910 & 925 & 3 & -6 & 6 & 576 & 573 & 1 & -4 & 6 & 584 & 551 & 6 & -2 & 6 & 848 & \\
\hline $7-11$ & 6 & 439 & 450 & -6 & -8 & 6 & 1231 & 1220 & 4 & -6 & 6 & 1297 & 1294 & 2 & -4 & 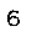 & 1231 & 1224 & 7 & -2 & 6 & 334 & \\
\hline$-6-11$ & 6 & 349 & 396 & -4 & -8 & 6 & 713 & 712 & 5 & -6 & 6 & 816 & 823 & 4 & -4 & 6 & 427 & 434 & 9 & -2 & 6 & 535 & \\
\hline$-4-1.1$ & 6 & 560 & 550 & -3 & -8 & 6 & 1339 & 1351 & 6 & -6 & 6 & 938 & 963 & 9 & -4 & 6 & 430 & 412 & -10 & -1 & 6 & 267 & \\
\hline$-1-11$ & 6 & 223 & 229 & -2 & -8 & 6 & 858 & 888 & 7 & -6 & 6 & 313 & 324 & -11 & -3 & 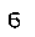 & 216 & 170 & -9 & -1 & 6 & 453 & \\
\hline $0-11$ & 6 & 529 & 524 & -1 & -8 & 6 & 1057 & 1073 & 8 & -6 & 6 & 687 & 681 & -9 & -3 & 6 & 870 & 852 & -8 & -1 & b & 199 & \\
\hline $1-11$ & 6 & 233 & 267 & 0 & -8 & E & 521 & 537 & 10 & -5 & 6 & 696 & 679 & -7 & -3 & 6 & 1860 & 1862 & -7 & -1 & 6 & 413 & \\
\hline $2-11$ & 6 & 216 & 250 & 1 & -8 & $\varepsilon$ & 899 & 878 & -9 & -5 & 6 & 658 & 664 & -6 & -3 & 6 & 378 & 398 & -6 & -1 & 6 & 278 & \\
\hline $3-11$ & 6 & 233 & 210 & 2 & -8 & 6 & 509 & 5.11 & -8 & -5 & 6 & 1.139 & 1145 & -5 & -3 & 6 & 1181 & 1.179 & -5 & -1 & 6 & 1072 & 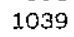 \\
\hline$-10-10$ & 6 & 64.8 & 633 & 3 & -8 & 6 & 1249 & 1245 & -7 & -5 & 6 & 524 & 543 & -4 & -3 & 6 & 952 & 940 & -4 & -1 & 6 & 706 & \\
\hline$-9-10$ & 6 & 537 & 538 & 5 & -8 & 6 & 557 & 554 & -6 & -5 & 6 & 502 & 487 & -3 & -3 & 5 & 1756 & 1698 & -3 & -1 & 6 & 164 & \\
\hline $8-10$ & 6 & 943 & 950 & -10 & -7 & 6 & 320 & 281 & -5 & -5 & 6 & 744 & 729 & -2 & -3 & 6 & 1834 & 1801 & -2 & -1 & 6 & 1973 & 15 \\
\hline$-7-10$ & 6 & 578 & 612 & -6 & -7 & $E$ & 541 & 525 & -3 & -5 & 6 & 1543 & 1519 & -1 & -3 & 6 & 2097 & 2119 & -1 & -1 & 6 & 224 & \\
\hline$-6-10$ & 6 & 858 & 853 & -3 & -7 & 6 & 939 & 919 & -2 & -5 & 6 & 657 & 657 & 0 & -3 & 6 & 1526 & 1546 & 0 & -1 & 6 & 2376 & 2 \\
\hline$-4-10$ & 6 & 958 & 945 & -2 & -7 & 6 & 300 & 292 & -1 & -5 & 6 & 919 & 883 & 1 & -3 & 6 & 819 & 820 & 1 & -1 & 6 & 288 & \\
\hline$-3-10$ & 6 & 220 & 228 & -1 & -7 & 6 & 1704 & 1769 & 0 & -5 & 6 & 325 & 352 & 2 & -3 & 6 & 1616 & 1575 & 2 & -1 & 6 & 1763 & 17 \\
\hline$-2-10$ & 6 & 991 & 1036 & 0 & -7 & 6 & 261 & 271 & 1 & -5 & 6 & 811 & 830 & 4 & -3 & 6 & 1382 & 1387 & 3 & -1 & 6 & 1633 & 16 \\
\hline $0-10$ & 6 & 213 & 197 & 1 & -7 & 6 & 1189 & 1190 & 2 & -5 & 6 & 496 & 463 & 5 & -3 & 6 & 199 & 232 & 4 & -1 & 6 & 1729 & 17 \\
\hline $1-10$ & 6 & 451 & 471 & 2 & -7 & 6 & 806 & 820 & 4 & -5 & 6 & 490 & 505 & 6 & -3 & 6 & 277 & 293 & 5 & -1 & 6 & 1250 & \\
\hline $5-10$ & 6 & 277 & 291 & 3 & -7 & 6 & 1084 & 1072 & 6 & -5 & 6 & 1078 & 1099 & -9 & -2 & 6 & 201 & 148 & 6 & -1 & 6 & 1380 & 142 \\
\hline$-11-9$ & 6 & 277 & 265 & 4 & -7 & 6 & 1040 & 1035 & 8 & -5 & 6 & 632 & 629 & -8 & -2 & 6 & 490 & 504 & 7 & -1 & 6 & 485 & \\
\hline$-10-9$ & 6 & 57.7 & 585 & 5 & -7 & 6 & 1006 & 997 & 9 & -5 & 6 & 324 & 347 & -7 & -2 & 6 & 1.503 & 1482 & 9 & -1 & 6 & 744 & \\
\hline$-8-9$ & 6 & 1212 & 1240 & 6 & -7 & 6 & 645 & 658 & -11 & -4 & 6 & 219 & 217 & -6 & -2 & 6 & 219 & 219 & 10 & -1 & 6 & 215 & \\
\hline$-7-9$ & 6 & 269 & 256 & 8 & -7 & 6 & 585 & 592 & -10 & -4 & 6 & 300 & 310 & -5 & -2 & 6 & 1338 & 1342 & 11 & -1 & 6 & 699 & \\
\hline$-6-9$ & 6 & 1163 & 1200 & -10 & -6 & 6 & 739 & 696 & -9 & -4 & 6 & 961 & 971 & -4 & -2 & 5 & 467 & 431. & -11 & 0 & 6 & 476 & \\
\hline $4-9$ & 6 & 1072 & 1075 & -9 & -6 & 6 & 311 & 306 & -8 & -4 & 6 & 891 & 913 & -3 & -2 & 6 & 1150 & 1139 & -10 & 0 & 6 & 292 & \\
\hline$-3-9$ & 6 & 861 & 851 & -8 & -6 & 6 & 769 & 771 & -7 & -4 & 6 & 1244 & 1219 & -2 & -2 & 6 & 2501 & 2459 & -9 & 0 & 6 & 727 & \\
\hline $2-9$ & 6 & 1186 & 1201 & -7 & -6 & $\mathrm{f}$ & 309 & 317 & -6 & -4 & 6 & 700 & 716 & -1 & -2 & 6 & 1421 & 1397 & -8 & 0 & 6 & 414 & \\
\hline
\end{tabular}




\begin{tabular}{|c|c|c|c|c|c|c|c|c|c|c|c|c|c|c|c|c|c|c|c|c|c|c|c|}
\hline 2 & 0 & 6 & 1349 & 1322 & -2 & 2 & 6 & 2254 & 2217 & 0 & 4 & 6 & 1204 & 1190 & -5 & 6 & 336 & 334 & -2 & 8 & & 631 & 28 \\
\hline 3 & 0 & 6 & 1306 & 1299 & -1 & 2 & 6 & 878 & 824 & 1 & 4 & 6 & 1744 & 1795 & -4 & 6 & 345 & 324 & -1 & 8 & 0 & 1533 & 1518 \\
\hline 4 & 0 & 6 & 765 & 773 & 0 & 2 & 6 & $141 B$ & 1388 & 2 & 4 & 6 & 281 & 217 & -3 & 6 & 755 & 785 & 1 & 8 & 6 & 318 & 321. \\
\hline 5 & 0 & 6 & 2188 & 2211 & 1 & 2 & 6 & 827 & 81.6 & 3 & 4 & 6 & 1750 & 1754 & -2 & 6 & 182 & 172 & 2 & 8 & 6 & 441 & 504 \\
\hline 6 & 0 & 6 & 947 & 959 & 3 & 2 & 6 & 1533 & 1474 & 4 & 4 & 6 & 1091 & 1115 & o & 6 & 637 & 670 & 3 & 8 & 6 & 469 & $\Delta 34$ \\
\hline 7 & 0 & 6 & 1086 & 1071 & 4 & 2 & 6 & 394 & 390 & 5 & 4 & 6 & 2160 & 2193 & 1 & 6 & 666 & 709 & 4 & 8 & 6 & 971 & 957 \\
\hline 8 & 0 & 6 & 284 & 249 & 5 & 2 & 6 & 344 & 344 & 6 & 4 & 6 & 286 & 306 & 4 & 6 & 61683 & 1681 & 5 & 8 & 6 & 283 & 236 \\
\hline 9 & 0 & 6 & 994 & 985 & 7 & 2 & 6 & 425 & 426 & 7 & 4 & 6 & 739 & 771 & 5 & 6 & 704 & 696 & 6 & 8 & 6 & 245 & 222 \\
\hline 10 & 0 & 6 & 655 & 642 & 9 & 2 & 6 & 198 & 228 & 8 & 4 & 6 & 731 & 750 & 6 & 6 & $6 \quad 1811$ & 1815 & 8 & 8 & 6 & 299 & 304 \\
\hline 11 & 0 & 6 & 824 & 824 & 10 & 2 & 6 & 650 & 667 & 9 & 4 & 6 & 194 & 218 & 7 & 6 & 447 & 471 & 11 & 8 & 6 & 462 & 458 \\
\hline-9 & 1 & 6 & 737 & 726 & 11 & 2 & 6 & 249 & 273 & 10 & 4 & 6 & 638 & 650 & 8 & 6 & 929 & 922 & -7 & 9 & 6 & 434 & 452 \\
\hline-8 & 1 & 6 & 649 & 669 & 12 & 2 & 6 & 536 & 522 & 11 & 4 & 6 & 268 & 252 & 9 & 6 & 385 & 398 & -5 & 9 & 6 & 654 & 636 \\
\hline-7 & 1 & 6 & 1035 & 1065 & -8 & 3 & 6 & 913 & 924 & -8 & 5 & 6 & 372 & 356 & 10 & 6 & 848 & 827 & -3 & 9 & 6 & 489 & 493 \\
\hline-6 & 1 & 6 & 757 & 766 & -6 & 3 & 6 & 1.217 & 1194 & -7 & 5 & 6 & 311 & 319 & 11 & 6 & 517 & 530 & -2 & 9 & 6 & 1121 & 1115 \\
\hline-4 & 1 & 6 & 1695 & 1683 & -4 & 3 & 6 & 1083 & 1035 & -6 & 5 & 6 & 558 & 564 & 12 & 6 & 756 & 771 & -1 & 9 & 6 & 1131 & 1100 \\
\hline-2 & 1 & 6 & 1852 & 1807 & -3 & 3 & 6 & 1765 & 1729 & -5 & 5 & 6 & 245 & 241 & -8 & 7 & 502 & 488 & 0 & 9 & 6 & 945 & 946 \\
\hline-1 & 1 & 6 & 920 & 871 & -2 & 3 & 6 & 1500 & 1472 & -4 & 5 & 6 & 810 & 800 & -7 & 7 & 782 & 745 & 1 & 9 & 6 & 499 & 517 \\
\hline 1 & 1 & 6 & 533 & 539 & -1 & 3 & 6 & 2138 & 2063 & -3 & 5 & 6 & 245 & 219 & -6 & 7 & 515 & 526 & 2 & 9 & 6 & 668 & 665 \\
\hline 2 & 1 & 6 & 1649 & 1614 & 0 & 3 & 6 & 2235 & 2274 & -1 & 5 & 6 & 1251 & 1224 & -5 & 7 & 607 & 594 & 3 & 9 & 6 & 661 & 639 \\
\hline 4 & 1 & 6 & 245 & 246 & 1 & 3 & 6 & 604 & 597 & 0 & 5 & 6 & 628 & 592 & -3 & 7 & 61227 & 1218 & 4 & 9 & 6 & 1494 & 1460 \\
\hline 5 & 1 & 6 & 1054 & 1116 & 3 & 3 & 6 & 2281 & 2294 & 1 & 5 & 6 & 1317 & 1387 & -1 & 7 & 61164 & 1134 & 5 & 9 & 6 & 476 & 482 \\
\hline 6 & 1 & 6 & 356 & 342 & 4 & 3 & 6 & 457 & 452 & 2 & 5 & 6 & 298 & 305 & 0 & 7 & 630 & 607 & 6 & 9 & 6 & 1053 & 1070 \\
\hline 7 & 1 & 6 & 1083 & 1104 & 5 & 3 & 6 & 1828 & 1838 & 3 & 5 & 6 & 1193 & 1167 & 1 & 7 & 395 & 386 & 7 & 9 & 6 & 424 & 425 \\
\hline 9 & 1 & 6 & 702 & 711 & 7 & 3 & 6 & 234 & 216 & 4 & 5 & 6 & 1564 & 1565 & 3 & 7 & 207 & 211 & 8 & 9 & 6 & 285 & 268 \\
\hline 10 & 1 & 6 & 782 & 778 & 8 & 3 & 6 & 186 & 194 & 5 & 5 & 6 & 1464 & 1496 & 5 & 7 & 442 & 467 & 9 & 9 & 6 & 244 & 244 \\
\hline 11 & 1 & 6 & 598 & 577 & 12 & 3 & 6 & 395 & 379 & 6 & 5 & 6 & 1202 & 1227 & 6 & 7 & 61200 & 1213 & -5 & 10 & 6 & 410 & 410 \\
\hline
\end{tabular}

Observed and calculated structure factors: $\left[\mathrm{OS}(\mathrm{CO})_{2} \mathrm{PPH}_{3}\right]_{2}(\mathrm{MU}-\mathrm{I})\left(\mathrm{MU}-\mathrm{PPH}_{2}\right)$

Page 18

$\begin{array}{rrrrrrrrrr}\mathrm{h} & \mathrm{k} & 1 & 10 \mathrm{FO} & 10 \mathrm{Fc} & \mathrm{h} & \mathrm{k} & \mathrm{l} & 1 \text { OFO } & 1 \text { OFc } \\ -2 & 10 & 6 & 857 & 831 & 11 & 12 & 6 & 581 & 559 \\ -1 & 10 & 6 & 369 & 356 & -3 & 13 & 6 & 261 & 225 \\ 0 & 10 & 6 & 1473 & 1457 & -2 & 13 & 6 & 567 & 526 \\ 1 & 10 & 6 & 459 & 466 & -1 & 13 & 6 & 248 & 218 \\ 2 & 10 & 6 & 1082 & 1150 & 0 & 13 & 6 & 468 & 486 \\ 3 & 10 & 6 & 386 & 414 & 1 & 13 & 6 & 221 & 157 \\ 4 & 10 & 6 & 1084 & 1110 & 5 & 13 & 6 & 391 & 392 \\ 5 & 10 & 6 & 924 & 905 & 7 & 13 & 6 & 626 & 620 \\ 6 & 10 & 6 & 1116 & 1146 & 10 & 13 & 6 & 233 & 310 \\ 7 & 10 & 6 & 707 & 696 & -2 & 14 & 6 & 492 & 453 \\ 8 & 10 & 6 & 504 & 519 & -1 & 14 & 6 & 390 & 327 \\ 9 & 10 & 6 & 579 & 555 & 0 & 14 & 6 & 548 & 532 \\ 10 & 10 & 6 & 307 & 318 & 1 & 14 & 6 & 268 & 293 \\ 11 & 10 & 6 & 422 & 419 & 3 & 14 & 6 & 483 & 477 \\ -5 & 11 & 6 & 215 & 235 & 5 & 14 & 6 & 327 & 331 \\ -1 & 11 & 6 & 278 & 242 & 6 & 14 & 6 & 236 & 211 \\ 0 & 11 & 6 & 913 & 898 & 0 & 15 & 6 & 382 & 418 \\ 2 & 11 & 6 & 870 & 869 & 1 & 15 & 6 & 425 & 389 \\ 3 & 11 & 6 & 445 & 474 & 3 & 15 & 6 & 348 & 348 \\ 4 & 11 & 6 & 668 & 671 & 4 & 15 & 6 & 338 & 318 \\ 5 & 11 & 6 & 1079 & 1090 & 5 & 15 & 6 & 665 & 660 \\ 5 & 11 & 6 & 824 & 839 & 7 & 15 & 6 & 435 & 446 \\ 7 & 11 & 6 & 828 & 798 & 3 & 16 & 6 & 293 & 325 \\ 8 & 11 & 6 & 482 & 450 & 4 & 16 & 6 & 399 & 397 \\ 9 & 11 & 6 & 610 & 633 & 5 & 16 & 6 & 435 & 433 \\ 11 & 11 & 6 & 700 & 690 & 6 & 16 & 6 & 548 & 513 \\ -3 & 12 & 6 & 271 & 238 & -4-14 & 7 & 417 & 421 \\ -2 & 12 & 6 & 332 & 304 & -3-14 & 7 & 509 & 516 \\ 5 & 12 & 6 & 789 & 788 & -2-14 & 7 & 454 & 437 \\ 6 & 12 & 6 & 346 & 340 & -1-14 & 7 & 463 & 482 \\ 7 & 12 & 6 & 871 & 820 & -6-13 & 7 & 478 & 474 \\ 8 & 12 & 6 & 241 & 207 & -5-13 & 7 & 250 & 266 \\ 9 & 12 & 6 & 527 & 494 & -3-13 & 7 & 750 & 756 \\ 10 & 12 & 6 & 409 & 425 & -1-13 & 7 & 887 & 902 \\ & & & & & & & & & \end{array}$

h $\mathrm{k} 110 \mathrm{Fo} 10 \mathrm{FC}$

h $\mathrm{k}$ I 10Fo 10Fc

h $\mathrm{k}$ I 10FO 1OFC

$\begin{array}{llll}1-13 & 7 & 486 & 464\end{array}$

$\begin{array}{lllll}-1 & -9 & 7 & 721 & 757\end{array}$

$\begin{array}{lllll}7 & -7 & 7 & 287 & 292\end{array}$

$\begin{array}{llll}-7-12 & 7 & 279 & 267\end{array}$

$\begin{array}{lllll}0 & -9 & 7 & 650 & 660\end{array}$

$\begin{array}{lllll}2 & -9 & 7 & 545 & 540\end{array}$

$\begin{array}{lllll}4 & -9 & 7 & 233 & 246\end{array}$

$\begin{array}{lllll}5 & -9 & 7 & 408 & 376\end{array}$

$\begin{array}{lllll}6 & -9 & 7 & 212 & 205\end{array}$

$\begin{array}{llll}-3-12 & 7 & 460 & 463\end{array}$

$\begin{array}{llll}-2-12 & 7 & 281 & 236\end{array}$

$\begin{array}{llll}-1-12 & 7 & 934 & 937\end{array}$

$\begin{array}{llll}1-12 & 7 & 721 & 747\end{array}$

$\begin{array}{llll}-12 & 7 & 358 & 337\end{array}$

$\begin{array}{llll}3-12 & 7 & 281 & 269\end{array}$

$\begin{array}{llll}-9-11 & 7 & 377 & 356\end{array}$

$\begin{array}{llll}-8-11 & 7 & 499 & 502\end{array}$

$\begin{array}{llll}-2-11 & 7 & 317 & 368\end{array}$

$\begin{array}{llll}-1-11 & 7 & 670 & 654\end{array}$

$\begin{array}{llll}1-11 & 7 & 715 & 717\end{array}$

$\begin{array}{llll}2-11 & 7 & 199 & 168\end{array}$

$\begin{array}{llll}4-11 & 7 & 697 & 673\end{array}$

$-10-10 \quad 7 \quad 395 \quad 364$

$\begin{array}{llll}-9-10 & 7 & 777 & 788\end{array}$

$\begin{array}{llll}-8-10 & 7 & 690 & 689\end{array}$

$\begin{array}{llll}-7-10 & 7 & 759 & 737\end{array}$

$\begin{array}{llll}-6-10 & 7 & 256 & 278\end{array}$

$\begin{array}{llll}-5-10 & 7 & 367 & 340\end{array}$

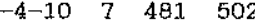

$\begin{array}{llll}-3-10 & 7 & 601 & 606\end{array}$

$\begin{array}{llll}-1-10 & 7 & 207 & 237\end{array}$

$\begin{array}{llll}1-10 & 7 & 296 & 272\end{array}$

$\begin{array}{lllll}-9 & -9 & 7 & 986 & 1023\end{array}$

$\begin{array}{lllll}-7 & -9 & 7 & 1307 & 1298\end{array}$

$\begin{array}{lllll}-6 & -9 & 7 & 286 & 275\end{array}$

$\begin{array}{lllll}-5 & -9 & 7 & 855 & 844\end{array}$

$\begin{array}{lllll}-4 & -9 & 7 & 501 & 526\end{array}$

$\begin{array}{lllll}-3 & -9 & 7 & 634 & 644\end{array}$

$\begin{array}{lllll}-9 & -8 & 7 & 619 & 655\end{array}$

$\begin{array}{lllll}-8 & -8 & 7 & 250 & 225\end{array}$

$\begin{array}{llllll}-7 & -8 & 7 & 1411 & 1426\end{array}$

$\begin{array}{lllll}-5 & -8 & 7 & 1145 & 1130\end{array}$

$\begin{array}{lllll}-4 & -8 & 7 & 660 & 651\end{array}$

$\begin{array}{lllll}-3 & -8 & 7 & 463 & 464\end{array}$

$\begin{array}{llllll}-2 & -8 & 7 & 1482 & 1571\end{array}$

$\begin{array}{lllll}-1 & -8 & 7 & 557 & 585\end{array}$

$\begin{array}{lllll}0 & -8 & 7 & 1252 & 1275\end{array}$

$\begin{array}{lllll}1 & -8 & 7 & 305 & 304\end{array}$

$\begin{array}{lllll}2 & -8 & 7 & 794 & 805\end{array}$

$\begin{array}{lllll}3 & -8 & 7 & 254 & 282\end{array}$

$\begin{array}{lllll}4 & -8 & 7 & 851 & 866\end{array}$

$\begin{array}{lllll}-10 & -7 & 7 & 238 & 235\end{array}$

$\begin{array}{lllll}-8 & -7 & 7 & 430 & 442\end{array}$

$\begin{array}{llllll}-7 & -7 & 7 & 853 & 868\end{array}$

$\begin{array}{lllll}-6 & -7 & 7 & 272 & 298\end{array}$

$\begin{array}{lllll}-5 & -7 & 7 & 825 & 802\end{array}$

$\begin{array}{lllll}-4 & -7 & 7 & 480 & 474\end{array}$

$\begin{array}{lllll}-3 & -7 & 7 & 238 & 224\end{array}$

$\begin{array}{lllll}-2 & -7 & 7 & 1729 & 1792\end{array}$

$\begin{array}{lllll}-1 & -7 & 7 & 176 & 129\end{array}$

$\begin{array}{lllll}0 & -7 & 7 & 1795 & 1831\end{array}$

$\begin{array}{lllll}2 & -7 & 7 & 730 & 718\end{array}$

$\begin{array}{lllll}3 & -7 & 7 & 887 & 880\end{array}$

$\begin{array}{lllll}4 & -7 & 7 & 1028 & 1038\end{array}$

$\begin{array}{lllll}5 & -7 & 7 & 674 & 641\end{array}$ $\begin{array}{lllll}-10 & -6 & 7 & 317 & 278\end{array}$

$\begin{array}{lllll}-9 & -6 & 7 & 321 & 342\end{array}$

$\begin{array}{lllll}-7 & -6 & 7 & 199 & 146\end{array}$

$\begin{array}{lllll}-6 & -6 & 7 & 227 & 192\end{array}$

$\begin{array}{llll}-6 & 7 & 224 & 212\end{array}$

$\begin{array}{lllll}-2 & -6 & 7 & 974 & 978\end{array}$

-1. $\begin{array}{llll}-6 & 7 & 403 & 435\end{array}$

$\begin{array}{lllll}0 & -6 & 7 & 2111 & 2201\end{array}$

$\begin{array}{lllll}1 & -6 & 7 & 405 & 391\end{array}$

$\begin{array}{lllll}2 & -5 & 7 & 912 & 89.1\end{array}$

$\begin{array}{lllll}3 & -6 & 7 & 1143 & 1165\end{array}$

$\begin{array}{lllll}4 & -6 & 7 & 432 & 443\end{array}$

$\begin{array}{lllll}5 & -6 & 7 & 1490 & 1464\end{array}$

$\begin{array}{lllll}6 & -6 & 7 & 463 & 452\end{array}$

$\begin{array}{lllll}7 & -6 & 7 & 821 & 852\end{array}$ $\begin{array}{llllll}-11 & -5 & 7 & 524 & 546\end{array}$ $\begin{array}{lllll}-10 & -5 & 7 & 265 & 314\end{array}$ $\begin{array}{lllll}-9 & -5 & 7 & 590 & 592\end{array}$ $\begin{array}{llllll}-8 & -5 & 7 & 608 & 579\end{array}$

$\begin{array}{lllll}-7 & -5 & 7 & 518 & 503\end{array}$

$\begin{array}{lllll}-6 & -5 & 7 & 290 & 301\end{array}$

$\begin{array}{lllll}-4 & -5 & 7 & 386 & 370\end{array}$

$\begin{array}{lllll}-2 & -5 & 7 & 399 & 386\end{array}$

$\begin{array}{lllll}-1 & -5 & 7 & 811 & 823\end{array}$

$\begin{array}{rrrrr}0 & -5 & 7 & 1467 & 1492\end{array}$

$\begin{array}{lllll}1 & -5 & 7 & 501 & 487\end{array}$

$\begin{array}{lllll}2 & -5 & 7 & 707 & 727\end{array}$

$\begin{array}{lllll}3 & -5 & 7 & 520 & 505\end{array}$

$\begin{array}{lllll}5 & -5 & 7 & 1353 & 1332\end{array}$

$\begin{array}{lllll}6 & -5 & 7 & 279 & 322\end{array}$

$\begin{array}{lllll}7 & -5 & 7 & 1141 & 1129\end{array}$

$\begin{array}{lllll}9 & -5 & 7 & 646 & 635\end{array}$

$\begin{array}{lllll}-2 & -9 & 7 & 958 & 1003\end{array}$ 


\begin{tabular}{|c|c|c|c|c|c|c|c|c|c|c|c|c|c|c|c|c|c|c|c|c|c|c|c|c|}
\hline-4 & -8 & 8 & 744 & 745 & 6 & -6 & 8 & 935 & 928 & 3 & -4 & 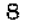 & 465 & 463 & 2 & -2 & 8 & 1150 & 1150 & $d$ & 4 & 0 & 457 & 452 \\
\hline-3 & -8 & 8 & 1043 & 1075 & 8 & -6 & 8 & 345 & 374 & 6 & -4 & 8 & 1265 & 1281 & 3 & -2 & 8 & 470 & 436 & 2 & 0 & 8 & 1655 & 1641 \\
\hline-2 & -8 & 8 & 345 & 341 & -10 & -5 & 8 & 290 & 241 & 7 & -4 & 8 & 385 & 393 & 4 & -2 & 8 & 1040 & 996 & 3 & 0 & 8 & 737 & 743 \\
\hline-1 & -8 & 8 & 1534 & 1557 & -9 & -5 & 8 & 647 & 619 & 8 & -4 & 8 & 924 & 959 & 5 & -2 & 8 & 476 & 447 & 4 & 0 & 8 & 358 & 385 \\
\hline 0 & -8 & 8 & 71.3 & 717 & -7 & -5 & 8 & 558 & 528 & 9 & -4 & 8 & 430 & 427 & 6 & -2 & 8 & 650 & 652 & 5 & 0 & 8 & 1665 & 1713 \\
\hline 1 & -8 & 8 & 684 & 704 & -6 & -5 & 8 & 558 & 555 & -11 & -3 & 8 & 487 & 521 & 7 & -2 & 8 & 338 & 312 & 6 & 0 & 8 & 337 & 362 \\
\hline 3 & -8 & 8 & 526 & 533 & -5 & -5 & 8 & 325 & 324 & -10 & -3 & 8 & 324 & 364 & 8 & -2 & 8 & 227 & 238 & 7 & 0 & 8 & 1037 & 1037 \\
\hline 5 & -8 & 8 & 305 & 304 & -4 & -5 & 8 & 268 & 234 & -9 & -3 & 8 & 782 & 791 & 9 & -2 & 8 & 260 & 256 & 9 & 0 & 8 & 481 & 491 \\
\hline 6 & -8 & 8 & 289 & 255 & -3 & -5 & 8 & 270 & 305 & -7 & -3 & 8 & 1518 & 1522 & -9 & -1 & 8 & 225 & 203 & 10 & 0 & 8 & 584 & 568 \\
\hline 10 & -7 & 8 & 421 & 408 & -2 & -5 & 8 & 407 & 416 & -5 & -3 & 8 & 1206 & 1210 & -8 & -1 & 3 & 321 & 319 & 11 & 0 & 8 & 506 & 499 \\
\hline-8 & -7 & 8 & 462 & 455 & -1 & -5 & 8 & 771 & 812 & -4 & -3 & 8 & 307 & 312 & -7 & -1 & 8 & 565 & 552 & -8 & 1 & 8 & 854 & 814 \\
\hline-7 & -7 & 8 & 770 & 762 & 0 & -5 & 8 & 1388 & 1392 & -3 & -3 & 8 & 350 & 344 & -6 & -1 & B & 1013 & 1010 & -7 & 1 & $B$ & 403 & 416 \\
\hline-6 & -7 & 8 & 1280 & 1280 & 1 & -5 & 8 & 1292 & 1290 & -2 & -3 & 8 & 1479 & 1483 & -5 & -1 & 8 & 1673 & 1631 & -6 & 1 & 8 & 545 & 542 \\
\hline-5 & -7 & 8 & 676 & 671 & 2 & -5 & 8 & 987 & 961 & -1 & -3 & 8 & 1051 & 1037 & -4 & -1 & 8 & 810 & 813 & -5 & 1 & 8 & 378 & 360 \\
\hline-4 & -7 & 8 & 956 & 935 & 3 & -5 & 8 & 443 & 416 & 0 & -3 & 8 & 995 & 983 & -3 & -1 & 8 & 658 & 672 & -4 & 1 & 8 & 395 & 395 \\
\hline-3 & $-\bar{t}$ & 8 & 930 & 937 & 4 & -5 & 8 & 1061 & 1026 & 2 & -3 & 8 & 214 & 201 & -2 & -1 & 8 & 1552 & 1508 & -3 & 1 & 8 & 277 & 250 \\
\hline-1 & -7 & 8 & 1694 & 1744 & 5 & -5 & 8 & 227 & 221 & 3 & -3 & 8 & 710 & 692 & -1 & -1 & 8 & 686 & 676 & -2 & 1 & 8 & 224 & 190 \\
\hline 1 & -7 & 8 & 1354 & 1355 & 6 & -5 & 8 & 1490 & 1537 & 4 & -3 & 8 & 293 & 303 & 0 & -1 & 8 & 3122 & 3132 & -1 & 1 & 8 & 727 & 712 \\
\hline 3 & -7 & 8 & 582 & 589 & 8 & -5 & 8 & 815 & 788 & 7 & -3 & 8 & 629 & 637 & 1 & -1 & 8 & 558 & 545 & 0 & 1 & 8 & 253 & 253 \\
\hline 4 & -7 & 8 & 678 & 688 & -10 & -4 & 8 & 458 & 461 & 8 & -3 & $B$ & 508 & 494 & 2 & -1 & 8 & 1985 & 1995 & 1 & 1 & 8 & 887 & 873 \\
\hline 5 & -7 & 8 & 398 & 439 & -9 & -4 & 8 & 817 & 778 & 9 & -3 & 8 & 505 & 514 & 3 & -1 & 8 & 340 & 359 & 2 & 1 & 8 & 1579 & 1580 \\
\hline 6 & -7 & 8 & 309 & 321 & -8 & -4 & 8 & 323 & 349 & 10 & -3 & 8 & 208 & 167 & 4 & -1 & 8 & 1197 & 1182 & 3 & 1 & 8 & 1020 & 1014 \\
\hline-9 & -6 & 8 & 367 & 329 & -7 & -4 & 8 & 1070 & 1033 & -11 & -2 & 8 & 494 & 482 & 5 & -1 & 8 & 1111 & 1141 & 5 & 1 & 8 & 1474 & 1478 \\
\hline-7 & -6 & 8 & 507 & 487 & -6 & -4 & 8 & 673 & 668 & -9 & -2 & 8 & 660 & 661 & 6 & -1 & 8 & 789 & 786 & 6 & 1 & 8 & 509 & 531 \\
\hline-5 & -6 & 8 & 775 & 778 & -5 & -4 & 8 & 603 & 575 & -8 & -2 & 8 & 285 & 305 & 7 & -1 & 8 & 350 & 370 & 7 & 1 & 8 & 1948 & 1978 \\
\hline-4 & -6 & 8 & 486 & 472 & -4 & -4 & 8 & 266 & 267 & -7 & -2 & 8 & 1640 & 1625 & 9 & -1 & 8 & 192 & 122 & 8 & 1 & 8 & 348 & 350 \\
\hline
\end{tabular}

\begin{tabular}{|c|c|c|c|c|c|c|c|c|c|c|c|c|c|c|c|c|c|c|c|c|c|c|c|c|}
\hline$h$ & $\mathrm{k}$ & 1 & 10EO & $10 \mathrm{HC}$ & $\mathrm{h}$ & $\mathrm{k}$ & 1 & $10 F O$ & $10 F c$ & $\mathrm{~h}$ & $\mathrm{k}$ & $l$ & 1OEO & $10 \mathrm{FC}$ & $\mathrm{h}$ & $\mathrm{k}$ & 1 & $10 \mathrm{FO}$ & $10 \mathrm{FC}$ & $\mathrm{h}$ & $\mathrm{k}$ & 1 & $10 F$ & $10 F C$ \\
\hline 9 & 1 & 8 & 888 & 884 & 4 & 3 & 8 & 1033 & 1030 & 4 & 5 & 8 & 785 & 801 & 8 & 7 & 8 & 950 & 911 & 10 & 9 & 8 & 243 & 247 \\
\hline 10 & 1 & 8 & 729 & 741 & 5 & 3 & 8 & 328 & 367 & 5 & 5 & 8 & 779 & 756 & 9 & 7 & 8 & 299 & 292 & 11 & 9 & 8 & 354 & 364 \\
\hline 11 & 1 & 8 & 470 & 452 & 6 & 3 & 8 & 388 & 366 & 6 & 5 & 8 & 1169 & 1217 & 10 & 7 & 8 & 513 & 530 & -6 & 10 & 8 & 291 & 299 \\
\hline-9 & 2 & 8 & 248 & 242 & 7 & 3 & 8 & 374 & 332 & 7 & 5 & 8 & 663 & 677 & 11 & 7 & 8 & 756 & 740 & -5 & 10 & 8 & 624 & 618 \\
\hline-8 & 2 & 8 & 936 & 939 & 8 & 3 & 8 & 729 & 747 & 8 & 5 & 8 & 403 & 410 & 12 & 7 & 8 & 447 & 405 & -4 & 10 & 8 & 412 & 418 \\
\hline-6 & 2 & 8 & 1413 & 1409 & 9 & 3 & 8 & 662 & 645 & 10 & 5 & 8 & 316 & 330 & -7 & 8 & 8 & 853 & 835 & -3 & 10 & 8 & 513 & 468 \\
\hline-5 & 2 & 8 & 519 & 503 & 10 & 3 & 8 & 416 & 408 & -5 & 6 & 8 & 614 & 621 & -5 & 8 & 8 & 759 & 760 & -2 & 10 & 8 & 744 & 756 \\
\hline-4 & 2 & 8 & 827 & 802 & 12 & 3 & 8 & 571 & 553 & -4 & 6 & 8 & 592 & 590 & -3 & 8 & 8 & 414 & 416 & 0 & 10 & 8 & 1150 & 1207 \\
\hline-3 & 2 & 8 & 315 & 286 & -8 & 4 & 8 & 464 & 476 & -3 & 6 & 8 & 358 & 374 & -2 & 8 & 8 & 442 & 444 & 2 & 10 & 8 & 737 & 775 \\
\hline-2 & 2 & 8 & 630 & 633 & -7 & 4 & 8 & 427 & 396 & -2 & 6 & 8 & 179 & 221 & -1 & 8 & 8 & 660 & 667 & 3 & 10 & 8 & 561 & 553 \\
\hline 1 & 2 & 8 & 1387 & 1412 & -6 & 4 & 8 & 1059 & 1069 & -1 & 6 & 8 & 660 & 665 & 0 & 8 & 8 & 241 & 187 & 4 & 10 & 8 & 415 & 390 \\
\hline 2 & 2 & 8 & 1407 & 1349 & -5 & 4 & 8 & 403 & 355 & 0 & 6 & 8 & 795 & 826 & 1 & 8 & 8 & 333 & 376 & 5 & 10 & 8 & 1040 & 1032 \\
\hline 3 & 2 & 8 & 937 & 901 & -4 & 4 & 8 & 1169 & 1182 & 1 & 6 & 8 & 1697 & 1762 & 2 & 8 & 8 & 637 & 690 & 5 & 10 & 8 & 694 & 688 \\
\hline 4 & 2 & 8 & 219 & 205 & -3 & 4 & 8 & 876 & 851 & 2 & 6 & 8 & 856 & 837 & 3 & 8 & 8 & 411 & 424 & 7 & 10 & 8 & 358 & 375 \\
\hline 5 & 2 & 8 & 396 & 371 & -2 & 4 & 8 & 298 & 279 & 3 & 6 & 8 & 1422 & 1453 & 4 & 8 & 8 & 329 & 337 & 8 & 10 & 8 & 457 & 475 \\
\hline 6 & 2 & 8 & 793 & 801 & -1 & 4 & 8 & 2090 & 2065 & 4 & 6 & 8 & 807 & 849 & 6 & 8 & 8 & 627 & 615 & -4 & 11 & 8 & 463 & 473 \\
\hline 7 & 2 & 8 & 1518 & 1532 & 0 & 4 & 8 & 547 & 561 & 5 & 6 & 8 & 756 & 761 & 7 & 8 & 8 & 685 & 674 & -3 & 11 & 8 & 369 & 358 \\
\hline 8 & 2 & 8 & 581 & 578 & 1 & 4 & 8 & 1808 & 1872 & 6 & 6 & 8 & 1815 & 1828 & 8 & 8 & 8 & 980 & 986 & -2 & 11 & 8 & 431 & 412 \\
\hline 9 & 2 & 8 & 860 & 844 & 2 & 4 & 8 & 369 & 344 & 8 & 6 & 8 & 702 & 698 & 9 & 8 & 8 & 434 & 433 & 0 & 11 & 8 & 1040 & 1056 \\
\hline 10 & 2 & 8 & 590 & 610 & 3 & 4 & 8 & 715 & 702 & 9 & 6 & 8 & 374 & 428 & 10 & 8 & 8 & 372 & 393 & 1 & 11 & 8 & 347 & 321 \\
\hline 11 & 2 & 8 & 260 & 233 & 4 & 4 & 8 & 1.196 & 1175 & 10 & 6 & 8 & 573 & 598 & 11 & 8 & 8 & 837 & 806 & 2 & 11 & 8 & 1312 & 1415 \\
\hline 12 & 2 & 8 & 717 & 720 & 5 & 4 & 8 & 1152 & 1136 & 11 & 6 & 8 & 515 & 498 & -6 & 9 & $B$ & 274 & 267 & 3 & 11 & $B$ & 243 & 262 \\
\hline-9 & 3 & 8 & 319 & 331 & 6 & 4 & 8 & 596 & 602 & 12 & 6 & 8 & 464 & 458 & -5 & 9 & $B$ & 1024 & 978 & 4 & 11 & 8 & 529 & 556 \\
\hline-8 & 3 & 8 & 741 & 745 & 7 & 4 & 8 & 720 & 713 & -7 & 7 & 8 & 587 & 602 & -3 & 9 & 8 & 586 & 600 & 5 & 11 & 8 & 1023 & 1024 \\
\hline-7 & 3 & 8 & 440 & 404 & $-B$ & 5 & 8 & 209 & 190 & -6 & 7 & 8 & 372 & 350 & -2 & 9 & $B$ & 402 & 395 & 6 & 11 & 8 & 518 & 508 \\
\hline-6 & 3 & 8 & 1735 & 1727 & -7 & 5 & 8 & 435 & 438 & -2 & 7 & 8 & 431 & 426 & -1 & 9 & 8 & 502 & 519 & 7 & 11 & 8 & 1030 & 1008 \\
\hline-4 & 3 & 8 & 1493 & 1478 & -6 & 5 & 8 & 496 & 491 & -1 & 7 & 8 & 218 & 219 & 0 & 9 & 8 & 802 & 839 & 8 & 11 & 8 & 614 & 589 \\
\hline-3 & 3 & 8 & 560 & 563 & -5 & 5 & 8 & 846 & 854 & 0 & 7 & 8 & 689 & 701 & 1 & 9 & $B$ & 645 & 669 & 9 & 11 & 8 & 434 & 453 \\
\hline-2 & 3 & 8 & 729 & 683 & -4 & 5 & 8 & 1173 & 1209 & 1 & 7 & 8 & 570 & 592 & 3 & 9 & 8 & 526 & 520 & -4 & 12 & 8 & 335 & 294 \\
\hline-1 & 3 & 8 & 1303 & 1280 & -3 & 5 & 8 & 628 & 614 & 2 & 7 & 8 & 1134 & 1122 & 5 & 9 & 8 & 330 & 329 & -3 & 12 & 8 & 261 & 295 \\
\hline 0 & 3 & 8 & 1158 & 1168 & -1 & 5 & 8 & 1725 & 1725 & 3 & 7 & 8 & 846 & 829 & 6 & 9 & 8 & 243 & 254 & -2 & 12 & 8 & 242 & 241 \\
\hline 1 & 3 & 8 & 307 & 325 & 0 & 5 & 8 & 829 & 807 & 4 & 7 & 8 & 834 & 812 & 7 & 9 & 8 & 386 & 378 & -1 & 12 & 8 & 228 & 242 \\
\hline 2 & 3 & 8 & 360 & 327 & 1 & 5 & 8 & 2789 & 2898 & 6 & 7 & 8 & 1342 & 1378 & 8 & 9 & 8 & 316 & 302 & 0 & 12 & 8 & 403 & 410 \\
\hline 3 & 3 & 8 & 146 & 144 & 3 & 5 & 8 & 1572 & 1571 & 7 & 7 & 8 & 432 & 423 & 9 & 9 & 8 & 371 & 386 & 1 & 12 & 8 & 666 & 676 \\
\hline
\end{tabular}




\begin{tabular}{|c|c|c|c|c|c|c|c|c|c|c|c|c|c|c|c|c|c|c|c|c|c|c|}
\hline-313 & 8 & 223 & 222 & $-2-11$ & 9 & 286 & 245 & 1 & -8 & 9 & 456 & 475 & -4 & -5 & 9 & 1084 & 1052 & 0 & -3 & 9 & 614 & 592 \\
\hline 113 & 8 & 418 & 424 & $-1-11$ & 9 & 688 & 700 & -9 & -7 & 9 & 547 & 556 & -2 & -5 & 9 & 975 & 978 & 1 & -3 & 9 & 613 & 613 \\
\hline 213 & 8 & 312 & 369 & $0-11$ & 9 & 631 & 636 & -8 & -7 & 9 & 349 & 357 & -1 & -5 & 9 & 909 & 916 & 2 & -3 & 9 & 245 & 255 \\
\hline 313 & 8 & 447 & 465 & $1-11$ & 9 & 755 & 769 & -7 & -7 & 9 & 932 & 926 & D & -5 & 9 & 1165 & 1.193 & 3 & -3 & 9 & 810 & 814 \\
\hline 413 & 8 & 410 & 416 & $2-11$ & 9 & 455 & 450 & -6 & -7 & 9 & 1083 & 1076 & 1 & -5 & 9 & 1251 & 1249 & 4 & -3 & 9 & 387 & 362 \\
\hline 513 & 8 & 259 & 287 & $-7-10$ & 9 & 348 & 367 & -5 & -7 & 9 & 1264 & 1259 & 2 & -5 & 9 & 1259 & 1274 & 5 & -3 & 9 & 312 & 306 \\
\hline 713 & 8 & 617 & 593 & $-6-10$ & 9 & 361 & 393 & -4 & -7 & 9 & 863 & 860 & 3 & -5 & 9 & 958 & 953 & 6 & -3 & 9 & 735 & 748 \\
\hline 813 & 8 & 327 & 330 & $-5-10$ & 9 & 229 & 251 & -3 & -7 & 9 & 473 & 474 & 5 & -5 & 9 & 1230 & 1260 & 7 & -3 & 9 & 982 & 983 \\
\hline 913 & 8 & 517 & 523 & $-3-10$ & 9 & 404 & 397 & -2 & -7 & 9 & 1322 & 1361 & 6 & -5 & 9 & 325 & 348 & 8 & -3 & 9 & 810 & 794 \\
\hline 1013 & 8 & 472 & 451 & $-1-10$ & 9 & 433 & 413 & -1 & -7 & 9 & 336 & 342 & 7 & -5 & 9 & 972 & 971 & 9 & -3 & 9 & 506 & 543 \\
\hline $\begin{array}{ll}-1 & 14\end{array}$ & 8 & 315 & 346 & $0-10$ & 9 & 693 & 699 & 0 & -7 & 9 & 1662 & 1680 & 9 & -5 & 9 & 395 & 378 & 10 & -3 & 9 & 484 & 488 \\
\hline 014 & 8 & 315 & 331 & $1-10$ & 9 & 568 & 569 & 1 & --7 & 9 & 269 & 302 & -8 & -4 & 9 & 652 & 636 & -10 & -2 & 9 & 792 & 786 \\
\hline 314 & 8 & 213 & 233 & $2-10$ & 9 & 641 & 616 & 2 & -7 & 9 & 944 & 941 & -6 & -4 & 9 & 768 & 778 & -9 & -2 & 9 & 211 & 189 \\
\hline 714 & $B$ & 278 & 224 & $3-10$ & 9 & 262 & 279 & 3 & -7 & 9 & 248 & 224 & -5 & -4 & 9 & 507 & 494 & -8 & -2 & 9 & 818 & 816 \\
\hline 814 & 8 & 329 & 287 & $4-10$ & 9 & 484 & 473 & 4 & -7 & 9 & 266 & 289 & -4 & -4 & 9 & 350 & 356 & -7 & -2 & 9 & 574 & 552 \\
\hline 914 & 8 & 284 & 303 & $\begin{array}{lll}-9 & -9\end{array}$ & 9 & 593 & 583 & 5 & -7 & 9 & 367 & 363 & -2 & -4 & 9 & 364 & 361 & -6 & -2 & 9 & 1482 & 1519 \\
\hline 115 & 8 & 402 & 409 & $\begin{array}{lll}-7 & -9\end{array}$ & 9 & 813 & 840 & 6 & -7 & 9 & 309 & 306 & -1 & -4 & 9 & 418 & 405 & -5 & -2 & 9 & 638 & 635 \\
\hline 416 & 8 & 226 & 217 & $-6-9$ & 9 & 215 & 179 & -9 & -6 & 9 & 460 & 438 & 1 & -4 & 9 & 1408 & 1395 & -4 & -2 & 9 & 1088 & 1092 \\
\hline 616 & 8 & 351 & 314 & $-5 \quad-9$ & 9 & 365 & 339 & -7 & -6 & 9 & 233 & 210 & 2 & -4 & 9 & 534 & 557 & -3 & -2 & 9 & 931 & 912 \\
\hline$-5-13$ & 9 & 550 & 528 & $-4-9$ & 9 & 313 & 325 & -6 & -6 & 9 & 1122 & 1108 & 3 & -4 & 9 & 885 & 881 & -1 & -2 & 9 & 2140 & 2146 \\
\hline$-4-13$ & 9 & 399 & 398 & $\begin{array}{ll}-2 & -9\end{array}$ & 9 & 745 & 778 & -5 & -6 & 9 & 650 & 619 & 5 & -4 & 9 & 917 & 921 & 0 & -2 & 9 & 294 & 303 \\
\hline$-3-13$ & 9 & 456 & 487 & $2-9$ & 9 & 598 & 610 & -4 & -6 & 9 & 1258 & 1228 & 6 & -4 & 9 & 743 & 763 & 1 & -2 & 9 & 1423 & 1413 \\
\hline$-1-13$ & 9 & 694 & 736 & $4-9$ & 9 & 229 & 242 & -3 & -6 & 9 & 732 & 707 & 7 & -4 & 9 & 1368 & 1360 & 2 & -2 & 9 & 231 & 209 \\
\hline$-7-1$ & 9 & 399 & 377 & $5-9$ & 9 & 259 & 251 & -2 & -6 & 9 & 1079 & 1111 & 8 & -4 & 9 & 519 & 525 & 4 & -2 & 9 & 356 & 371 \\
\hline & 9 & 226 & 253 & $6-9$ & 9 & 324 & 357 & -1 & -6 & 9 & 558 & 599 & 9 & -4 & 9 & 635 & 622 & 5 & -2 & 9 & 256 & 248 \\
\hline & 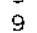 & & & ? & & - & ( & 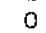 & -6 & 9 & 1503 & 1586 & -10 & -3 & 9 & 573 & 581 & 8 & -2 & 9 & 463 & 460 \\
\hline
\end{tabular}

Observed ard calculated structure factors: $\quad$ LOS(CO) $\left.\mathrm{PPH}_{3}\right]_{2}$ (MU-I) (MU-PPH $)$

Page 24

$\mathrm{h} k \mathrm{k}$ 10FO 10FC $\mathrm{h} k \mathrm{k}$ 10Fo 10FC

$\begin{array}{lllll}9 & -2 & 9 & 326 & 284\end{array}$

$\begin{array}{lllll}10 & -2 & 9 & 408 & 385\end{array}$

$\begin{array}{lllll}-10 & -1 & 9 & 614 & 645\end{array}$

$\begin{array}{lllll}-8 & -1 & 9 & 553 & 518\end{array}$

$\begin{array}{lllll}-7 & -1 & 9 & 687 & 706\end{array}$

$\begin{array}{lllll}-6 & -1 & 9 & 1382 & 1367\end{array}$

$\begin{array}{lllll}-5 & -1 & 9 & 1343 & 1311\end{array}$

$\begin{array}{lllll}-4 & -1 & 9 & 1299 & 1290\end{array}$

$\begin{array}{lllll}-3 & -1 & 9 & 1380 & 1370\end{array}$

$\begin{array}{lllll}-2 & -1 & 9 & 307 & 317\end{array}$

$\begin{array}{lllll}-1 & -1 & 9 & 2125 & 2173\end{array}$

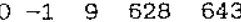

$\begin{array}{lllll}1 & -1 & 9 & 3068 & 3087\end{array}$

$\begin{array}{lllll}2 & -1 & 9 & 609 & 572\end{array}$

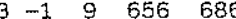

$\begin{array}{lllll}4 & -1 & 9 & 624 & 606\end{array}$

$\begin{array}{lllll}5 & -1 & 9 & 422 & 437\end{array}$

$\begin{array}{llll}-1 & 9 & 668 & 661\end{array}$

$\begin{array}{lllll}7 & -1 & 9 & 253 & 279\end{array}$

$\begin{array}{lllll}9 & -1 & 9 & 207 & 130\end{array}$

$\begin{array}{rrrrr}-10 & 0 & 9 & 318 & 330\end{array}$

$\begin{array}{lllll}-8 & 0 & 9 & 235 & 213\end{array}$

$\begin{array}{lllll}-6 & 0 & 9 & 390 & 358\end{array}$

$\begin{array}{lllll}-5 & 0 & 9 & 1425 & 1433\end{array}$

$\begin{array}{lllll}-4 & 0 & 9 & 844 & 850\end{array}$

$\begin{array}{lllll}-3 & 0 & 9 & 997 & 967\end{array}$

$\begin{array}{lllll}-1 & 0 & 9 & 762 & 830\end{array}$

o 0918041790

10928492855

200912641251

30917001702

409999995

$\begin{array}{llllll}5 & 0 & 9 & 348 & 344\end{array}$

$6 \quad 0 \quad 910801094$ $\begin{array}{lllll}8 & 0 & 9 & 847 & 841\end{array}$

$\begin{array}{lllll}10 & 0 & 9 & 326 & 261\end{array}$

$\begin{array}{lllll}11 & 0 & 9 & 243 & 229\end{array}$

$\begin{array}{lllll}-9 & 1 & 9 & 259 & 188\end{array}$

$\begin{array}{lllll}-7 & 1 & 9 & 460 & 472\end{array}$

$\begin{array}{lllll}-6 & 1 & 9 & 229 & 225\end{array}$

$\begin{array}{lllll}-5 & 1 & 9 & 654 & 637\end{array}$

$\begin{array}{lllll}-3 & 1 & 9 & 1154 & 1170\end{array}$

$\begin{array}{lllll}-1 & 1 & 9 & 369 & 377\end{array}$

$\begin{array}{lllll}0 & 1 & 9 & 1415 & 1457\end{array}$

$\begin{array}{lllll}1 & 1 & 9 & 810 & 826\end{array}$

$\begin{array}{lllll}2 & 1 & 1308 & 1324\end{array}$

$\begin{array}{lllll}3 & 1 & 9 & 1088 & 1076\end{array}$

41913021323

$\begin{array}{lllll}5 & 1 & 9 & 319 & 318\end{array}$

$6 \quad 1 \quad 9 \quad 16331633$

$\begin{array}{lllll}7 & 1 & 9 & 544 & 526\end{array}$

$8 \quad 1 \quad 9 \quad 1174 \quad 1164$

$\begin{array}{lllll}9 & 1 & 9 & 314 & 311\end{array}$

$\begin{array}{lllll}10 & 1 & 9 & 427 & 458\end{array}$

$\begin{array}{lllll}11 & 1 & 9 & 629 & 613\end{array}$

$\begin{array}{lllll}-10 & 2 & 9 & 350 & 366\end{array}$

$\begin{array}{lllll}-9 & 2 & 9 & 396 & 377\end{array}$

$\begin{array}{lllll}-7 & 2 & 9 & 972 & 974\end{array}$

$\begin{array}{lllll}-5 & 2 & 9 & 1088 & 1078\end{array}$

$\begin{array}{lllll}-4 & 2 & 9 & 681 & 673\end{array}$

$\begin{array}{lllll}-3 & 2 & 9 & 441 & 424\end{array}$

$\begin{array}{lllll}-2 & 2 & 9 & 551 & 524\end{array}$

$\begin{array}{lllll}-1 & 2 & 9 & 351 & 293\end{array}$

$\begin{array}{lllll}0 & 2 & 9 & 786 & 778\end{array}$

$\begin{array}{lllll}1 & 2 & 9 & 440 & 416\end{array}$

$2291710 \quad 1715$

$\begin{array}{lllll}3 & 2 & 9 & 535 & 554\end{array}$

42911911196 $\mathrm{h} \mathrm{k} l$ 10Fo 10FC

$\begin{array}{lllll}5 & 2 & 9 & 664 & 656\end{array}$

$\begin{array}{lllll}6 & 2 & 9 & 1286 & 1314\end{array}$

$\begin{array}{lllll}7 & 2 & 9 & 1298 & 1303\end{array}$

$\begin{array}{lllll}8 & 2 & 9 & 1305 & 1312\end{array}$

$\begin{array}{lllll}9 & 2 & 9 & 767 & 747\end{array}$

$\begin{array}{lllll}10 & 2 & 9 & 562 & 544\end{array}$

$\begin{array}{lllll}11 & 2 & 9 & 706 & 705\end{array}$

$\begin{array}{lllll}-9 & 3 & 9 & 456 & 454\end{array}$

$\begin{array}{lllll}-7 & 3 & 9 & 992 & 1010\end{array}$

$\begin{array}{lllll}-6 & 3 & 9 & 259 & 214\end{array}$

$\begin{array}{lllll}-5 & 3 & 9 & 1551 & 1575\end{array}$

$\begin{array}{lllll}-4 & 3 & 9 & 327 & 324\end{array}$

$\begin{array}{lllll}-3 & 3 & 9 & 471 & 491\end{array}$

$\begin{array}{lllll}-2 & 3 & 9 & 431 & 428\end{array}$

$\begin{array}{lllll}0 & 3 & 9 & 209 & 178\end{array}$

$\begin{array}{lllll}2 & 3 & 9 & 440 & 427\end{array}$

$\begin{array}{lllll}3 & 3 & 9 & 825 & 776\end{array}$

$\begin{array}{lllll}4 & 3 & 9 & 586 & 579\end{array}$

$\begin{array}{lllll}6 & 3 & 9 & 405 & 401\end{array}$

$\begin{array}{lllll}7 & 3 & 9 & 1126 & 1141\end{array}$

$\begin{array}{lllll}8 & 3 & 9 & 919 & 931\end{array}$

$\begin{array}{lllll}9 & 3 & 9 & 766 & 805\end{array}$

$\begin{array}{lllll}10 & 3 & 9 & 529 & 499\end{array}$

$\begin{array}{lllll}11 & 3 & 9 & 564 & 563\end{array}$

$-9 \quad 4 \quad 9590 \quad 563$

$\begin{array}{lllll}-7 & 4 & 9 & 606 & 625\end{array}$

$\begin{array}{lllll}-6 & 4 & 9 & 820 & 788\end{array}$

$\begin{array}{lllll}-5 & 4 & 9 & 1061 & 1049\end{array}$

$\begin{array}{lllll}-4 & 4 & 9 & 571 & 564\end{array}$

$\begin{array}{lllll}-3 & 4 & 9 & 867 & 866\end{array}$

$\begin{array}{lllll}-2 & 4 & 9 & 982 & 990\end{array}$

o 44917251765

$\begin{array}{lllll}1 & 4 & 9 & 234 & 227\end{array}$

$\begin{array}{lllll}2 & 4 & 9 & 906 & 892\end{array}$ h $\mathrm{k}$ l $10 \mathrm{FO}$ IOFC

$349383 \quad 376$

$\begin{array}{lllll}4 & 4 & 9 & 179 & 137\end{array}$

$\begin{array}{lllll}5 & 4 & 9 & 754 & 798\end{array}$

$\begin{array}{lllll}6 & 4 & 9 & 637 & 625\end{array}$

$\begin{array}{lllll}7 & 4 & 9 & 172 & 72\end{array}$

949649680

$\begin{array}{lllll}10 & 4 & 9 & 297 & 314\end{array}$

$\begin{array}{lllll}11 & 4 & 9 & 383 & 397\end{array}$

$\begin{array}{lllll}-7 & 5 & 9 & 374 & 423\end{array}$

$\begin{array}{lllll}-6 & 5 & 9 & 680 & 691\end{array}$

$\begin{array}{lllll}-5 & 5 & 9 & 620 & 642\end{array}$

$\begin{array}{lllll}-4 & 5 & 9 & 785 & 753\end{array}$

$\begin{array}{lllll}-3 & 5 & 9 & 647 & 526\end{array}$

$\begin{array}{lllll}-2 & 5 & 9 & 981 & 981\end{array}$

$\begin{array}{lllll}-1 & 5 & 9 & 608 & 599\end{array}$

$\begin{array}{lllll}0 & 5 & 9 & 2189 & 2271\end{array}$

$\begin{array}{lllll}1 & 5 & 9 & 731 & 756\end{array}$

$\begin{array}{lllll}2 & 5 & 9 & 1858 & 1932\end{array}$

$\begin{array}{lllll}3 & 5 & 9 & 670 & 663\end{array}$

$459573 \quad 578$

$5 \quad 5 \quad 913861398$

$\begin{array}{lllll}6 & 5 & 9 & 621 & 618\end{array}$

$\begin{array}{lllll}7 & 5 & 9 & 959 & 950\end{array}$

$\begin{array}{lllll}8 & 5 & 9 & 643 & 647\end{array}$

$\begin{array}{lllll}-6 & 6 & 9 & 504 & 498\end{array}$

$\begin{array}{lllll}-4 & 6 & 9 & 1097 & 1117\end{array}$

$\begin{array}{lllll}-3 & 6 & 9 & 475 & 486\end{array}$

$\begin{array}{lllll}6 & 9 & 804 & 823\end{array}$

$\begin{array}{lllll}-1 & 6 & 9 & 537 & 530\end{array}$

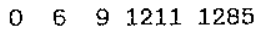

$\begin{array}{lllll}1 & 6 & 9 & 1447 & 1502\end{array}$

$\begin{array}{lllll}2 & 6 & 9 & 2254 & 2274\end{array}$

$\begin{array}{llllr}3 & 6 & 9 & 606 & 597\end{array}$

$\begin{array}{lllll}4 & 6 & 9 & 1068 & 1071\end{array}$ h $\mathrm{k} \perp 10 \mathrm{FO}$ 10FC

$\begin{array}{lllll}5 & 6 & 9 & 1218 & 1216\end{array}$

$\begin{array}{rrrrr}6 & 6 & 9 & 182 & 61 \\ 7 & 6 & 9 & 1195 & 1195\end{array}$

$\begin{array}{lllll}8 & 6 & 9 & 292 & 204\end{array}$

$\begin{array}{lllll}9 & 6 & 9 & 559 & 542\end{array}$

$\begin{array}{lllll}10 & 6 & 9 & 257 & 218\end{array}$

$\begin{array}{lllll}11 & 6 & 9 & 269 & 241\end{array}$

$\begin{array}{lllll}12 & 6 & 9 & 214 & 204\end{array}$

$\begin{array}{lllll}-4 & 7 & 9 & 628 & 637\end{array}$

$\begin{array}{lllll}-3 & 7 & 9 & 315 & 330\end{array}$

$\begin{array}{lllll}-2 & 7 & 9 & 506 & 482\end{array}$

$\begin{array}{lllll}1 & 7 & 9 & 1073 & 1110\end{array}$

$\begin{array}{lllll}2 & 7 & 9 & 923 & 954\end{array}$

$\begin{array}{lllll}3 & 7 & 9 & 1321 & 1330\end{array}$

$\begin{array}{lllll}4 & 7 & 9 & 837 & 828\end{array}$

$\begin{array}{lllll}5 & 7 & 9 & 1230 & 1238\end{array}$

$\begin{array}{lllll}6 & 7 & 9 & 427 & 426\end{array}$

$\begin{array}{lllll}7 & 7 & 9 & 1077 & 1076\end{array}$

$\begin{array}{lllll}8 & 7 & 9 & 402 & 381\end{array}$

$\begin{array}{lllll}9 & 7 & 9 & 623 & 638\end{array}$

$\begin{array}{lllll}10 & 7 & 9 & 503 & 479\end{array}$

$\begin{array}{lllll}11 & 7 & 9 & 251 & 259\end{array}$

$\begin{array}{lllll}12 & 7 & 9 & 659 & 674\end{array}$

$\begin{array}{lllll}-6 & 8 & 9 & 676 & 675\end{array}$

$\begin{array}{lllll}-4 & 8 & 9 & 247 & 236\end{array}$

$\begin{array}{lllll}-2 & 8 & 9 & 370 & 362\end{array}$

$\begin{array}{lllll}-1 & 8 & 9 & 422 & 460\end{array}$

$\begin{array}{lllll}0 & 8 & 9 & 310 & 295\end{array}$

$\begin{array}{lllll}1 & 8 & 9 & 360 & 362\end{array}$

$\begin{array}{lllll}2 & 8 & 9 & 233 & 247\end{array}$

$\begin{array}{lllll}3 & 8 & 9 & 940 & 942\end{array}$

$\begin{array}{lllll}5 & 8 & 9 & 681 & 668\end{array}$

$\begin{array}{lllll}6 & 8 & 9 & 535 & 532\end{array}$

$\begin{array}{lllll}7 & 8 & 9 & 724 & 736\end{array}$ 


\begin{tabular}{|c|c|c|c|c|c|c|c|c|c|c|c|c|c|c|c|c|c|c|c|c|c|c|c|}
\hline-2 & 9 & 9 & 197 & 189 & 7 & 11 & 9 & 553 & 575 & $-5-12$ & 10 & 445 & 435 & -3 & -8 & 10 & 569 & 595 & 8 & -6 & 10 & 212 & 68 \\
\hline-1 & 9 & 9 & 439 & 484 & 8 & 11 & 9 & 579 & 535 & $-4-12$ & 10 & 766 & 785 & -2 & -8 & 10 & 202 & 226 & -10 & -5 & 10 & 226 & 202 \\
\hline 1 & 9 & 9 & 376 & 384 & -3 & 12 & 9 & 516 & 493 & $-3-12$ & 10 & 264 & 253 & -1 & -8 & 10 & 857 & 885 & -8 & -5 & 10 & 354 & 327 \\
\hline 2 & 9 & 9 & 255 & 287 & -1 & 12 & 9 & 312 & 317 & $-2-12$ & 10 & 665 & 657 & 0 & -8 & 10 & 331 & 319 & -6 & -5 & 10 & 379 & 350 \\
\hline 3 & 9 & 9 & 355 & 374 & 0 & 12 & 9 & 438 & 456 & $-1-12$ & 10 & 288 & 280 & 2 & -8 & 10 & 393 & 366 & -5 & -5 & 10 & 1108 & 1078 \\
\hline 6 & 9 & 9 & 290 & 288 & 1 & 12 & 9 & 624 & 610 & $0-12$ & 10 & 682 & 700 & 3 & -8 & 10 & 454 & 432 & -3 & -5 & 10 & 1452 & 1511 \\
\hline 7 & 9 & 9 & 266 & 260 & 2 & 12 & 9 & 888 & 879 & $-6-11$ & 10 & 507 & 493 & 5 & -8 & 10 & 323 & 265 & -2 & -5 & 10 & 192 & 151 \\
\hline 8 & 9 & 9 & 1019 & 1019 & 3 & 12 & 9 & 938 & 966 & $-4-11$ & 10 & 890 & 911 & 6 & -8 & 10 & 306 & 331 & -1 & -5 & 10 & 990 & 1012 \\
\hline 9 & 9 & 9 & 606 & 603 & 4 & 12 & 9 & 571 & 565 & $-2-11$ & 10 & 664 & 643 & -10 & -7 & 10 & 852 & 844 & 0 & -5 & 10 & 1109 & 1119 \\
\hline 10 & 9 & 9 & 636 & 634 & 6 & 12 & 9 & 797 & 805 & $-1-11$ & 10 & 449 & 423 & -8 & -7 & 10 & 727 & 760 & 1 & -5 & 10 & 925 & 915 \\
\hline 12 & 9 & 9 & 614 & 604 & 8 & 12 & 9 & 835 & 821 & $0-11$ & 10 & 514 & 556 & -7 & -7 & 10 & 522 & 537 & 2 & -5 & 10 & 1155 & 1178 \\
\hline-5 & 10 & 9 & 546 & 561 & 10 & 12 & 9 & 419 & 421 & $1-11$ & 10 & 748 & 720 & -6 & -7 & 10 & 850 & 862 & 3 & -5 & 10 & 641 & 659 \\
\hline-4 & 10 & 9 & 939 & 945 & -1 & 13 & 9 & 218 & 136 & $2-11$ & 10 & 467 & 456 & -5 & -7 & 10 & 1162 & 1156 & 4 & -5 & 10 & 992 & 985 \\
\hline-3 & 10 & 9 & 395 & 378 & 0 & 13 & 9 & 238 & 252 & $3-11$ & 10 & 523 & 536 & -4 & -7 & 10 & 957 & 983 & 5 & -5 & 10 & 329 & 298 \\
\hline-2 & 10 & 9 & 516 & 520 & 2 & 13 & 9 & 703 & 720 & $-5-10$ & 10 & 366 & 360 & -3 & -7 & 10 & 859 & 891 & 6 & -5 & 10 & 949 & 355 \\
\hline-1 & 10 & 9 & 587 & 583 & 3 & 13 & 9 & 467 & 475 & $-4-10$ & 10 & 641 & 652 & -2 & -7 & 10 & 244 & 274 & 7 & -5 & 10 & 387 & 337 \\
\hline 1 & 10 & 9 & 846 & 873 & 8 & 13 & 9 & 496 & 463 & $-2-10$ & 10 & 517 & 523 & -1 & -7 & 10 & 1215 & 1232 & 8 & -5 & 10 & 641 & 633 \\
\hline 3 & 10 & 9 & 311 & 352 & 6 & 13 & 9 & 542 & 514 & $-1-10$ & 10 & 409 & 393 & 1 & -7 & 10 & 1095 & 1105 & -10 & -4 & 10 & 259 & 305 \\
\hline 4 & 10 & 9 & 561 & 553 & 7 & 13 & 9 & 218 & 267 & $0-10$ & 10 & 321 & 328 & 2 & -7 & 10 & 211 & 225 & -9 & -4 & 10 & 302 & 261 \\
\hline 6 & 10 & 9 & 382 & 385 & 8 & 13 & 9 & 728 & 697 & $1-10$ & 10 & 1033 & 986 & 3 & -7 & 10 & 263 & 277 & -8 & -4 & 10 & 266 & 257 \\
\hline 7 & 10 & 9 & 321 & 319 & 9 & 13 & 9 & 393 & 384 & $2-10$ & 10 & 457 & 433 & -10 & -6 & 10 & 614 & 600 & -7 & -4 & 10 & 764 & 75 \\
\hline 8 & 10 & 9 & 349 & 364 & 10 & 13 & 9 & 426 & 443 & $3-10$ & 10 & 652 & 646 & -8 & -6 & 10 & 481 & 477 & -6 & -4 & 10 & 343 & 358 \\
\hline 9 & 10 & 9 & 229 & 203 & 0 & 14 & 9 & 279 & 257 & $\begin{array}{ll}-9 & -9\end{array}$ & 10 & 276 & 248 & -7 & -6 & 10 & 652 & 642 & -4 & -4 & 10 & 599 & 602 \\
\hline 10 & 10 & 9 & 460 & 452 & 2 & 14 & 9 & 310 & 305 & $\begin{array}{lll}-8 & -9\end{array}$ & 10 & 274 & 279 & -6 & -6 & 10 & 451 & 479 & -3 & -4 & 10 & 964 & 971 \\
\hline-4 & 11 & 9 & 354 & 353 & 4 & 14 & 9 & 489 & 460 & $-6-9$ & 10 & 477 & 445 & -5 & -6 & 10 & 1554 & 1544 & -2 & -4 & 10 & 383 & 385 \\
\hline-3 & 11 & 9 & 444 & 460 & 6 & 14 & 9 & 280 & 240 & $-2 \quad-9$ & 10 & 278 & 283 & -4 & -6 & 10 & 834 & 845 & -1 & -4 & 10 & 512 & 48 \\
\hline
\end{tabular}

\begin{tabular}{|c|c|c|c|c|c|c|c|c|c|c|c|c|c|c|c|c|c|c|c|c|c|c|c|c|}
\hline \multicolumn{4}{|c|}{ observed and } & \multicolumn{2}{|c|}{ calculated } & \multicolumn{4}{|c|}{ structure factors: } & \multicolumn{10}{|c|}{$\left[\mathrm{OS}(\mathrm{CO})_{2} \mathrm{PPH}_{3}\right]_{2}(\mathrm{MU}-\mathrm{I}) \quad\left(\mathrm{MU}-\mathrm{PPH}_{2}\right)$} & \multicolumn{5}{|c|}{ Page 26} \\
\hline $\mathrm{h}$ & $\mathrm{k}$ & 1 & $10 \mathrm{FO}$ & $10 \mathrm{FC}$ & 12 & $\mathrm{k}$ & 1. & 1OFO & $10 \mathrm{CC}$ & $\mathbf{h}$ & $\mathrm{k}$ & 1 & $10 \mathrm{FO}$ & 10FC & h & $\mathrm{k}$ & 1 & $10 F O$ & $10 \mathrm{FC}$ & $h$ & k & 1 & $10 F O$ & 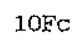 \\
\hline 0 & -4 & 10 & 919 & 953 & -1 & -2 & 10 & 316 & 355 & 1 & 0 & 10 & 1611 & 1658 & 7 & 2 & 10 & 1112 & 1091 & 4 & 4 & 10 & 218 & 212 \\
\hline 1 & -4 & 10 & 218 & 193 & 0 & -2 & 10 & 1380 & 1404 & 2 & 0 & 10 & 2579 & 2605 & 8 & 2 & 10 & 855 & 880 & 5 & 4 & 30 & 275 & 298 \\
\hline 2 & -4 & 10 & 1421 & 1449 & 1 & -2 & 10 & 189 & 180 & 3 & 0 & 10 & 647 & 622 & 9 & 2 & 10 & 719 & 701 & 6 & 4 & 10 & 261 & 291 \\
\hline 3 & -4 & 10 & 403 & 389 & 2 & -2 & 10 & 195 & 191 & 4 & 0 & 10 & 649 & 660 & 10 & 2 & 10 & 636 & 647 & 8 & 4 & 10 & 1013 & 1005 \\
\hline 4 & -4 & 10 & 1061 & 1062 & 3 & -2 & 10 & 478 & 469 & 5 & 0 & 10 & 743 & 739 & -9 & 3 & 10 & 331 & 313 & 9 & 4 & 10 & 328 & 308 \\
\hline 5 & -4 & 10 & 589 & 612 & 4 & -2 & 10 & 714 & 686 & 7 & 0 & 10 & 790 & 801 & -8 & 3 & 10 & 538 & 528 & 10 & 4 & 10 & 712 & 714 \\
\hline 6 & -4 & 10 & 1145 & 1162 & 7 & -2 & 10 & 686 & 673 & -9 & 1 & 10 & 265 & 281 & -7 & 3 & 10 & 285 & 299 & 11 & 4 & 10 & 241 & 246 \\
\hline 7 & -4 & 10 & 717 & 715 & 9 & -2 & 10 & 828 & 823 & -4 & 1 & 10 & 1374 & 1375 & -6 & 3 & 10 & 974 & 973 & 12 & 4 & 10 & 457 & 468 \\
\hline 8 & -4 & 10 & 1052 & 1043 & -9 & -1 & 10 & 924 & 914 & -2 & 1 & 10 & 1208 & 1216 & -4 & 3 & 10 & 921 & 917 & -8 & 5 & 10 & 654 & 6.39 \\
\hline 9 & -4 & 10 & 295 & 304 & -7 & -1 & 10 & 746 & 741 & -1 & 1 & 10 & 267 & 274 & -3 & 3 & 10 & 504 & 503 & -7 & 5 & 10 & 227 & 231 \\
\hline-10 & -3 & 10 & 625 & 612 & -6 & -1 & 10 & 931 & 900 & 0 & 1 & 10 & 462 & 486 & -2 & 3 & 10 & 513 & 519 & -6 & 5 & 10 & 269 & 313 \\
\hline-9 & -3 & 10 & $\Delta 11$ & 422 & -5 & -1 & 10 & 1136 & 1161 & 1 & 1 & 10 & 1779 & 1832 & -1 & 3 & 10 & 729 & 691 & -5 & 5 & 10 & 990 & 983 \\
\hline-7 & -3 & 10 & 616 & 632 & -4 & -1 & 10 & 1567 & 1551 & 2 & 1. & 10 & 1298 & 1338 & 1 & 3 & 10 & 203 & 186 & -4 & 5 & 10 & 451 & 445 \\
\hline-5 & -3 & 10 & 891 & 879 & -3 & -1 & 10 & 760 & 742 & 3 & 1 & 10 & 1836 & 1821 & 2 & 3 & 10 & 818 & 811 & -3 & 5 & 10 & 1201 & 1204 \\
\hline-4 & -3 & 10 & 465 & 478 & -2 & -1 & 10 & 1166 & 1163 & 4 & 1 & 10 & 888 & 878 & 3 & 3 & 10 & 1008 & 1027 & -2 & 5 & 10 & 233 & 278 \\
\hline-3 & -3 & 10 & 419 & 436 & -1 & -1 & 10 & 862 & 898 & 5 & 1 & 10 & 1091 & 1075 & 5 & 3 & 10 & 961 & 966 & -1 & 5 & 10 & 944 & 989 \\
\hline-2 & -3 & 10 & 313 & 291 & 0 & -1 & 10 & 1731 & 1747 & 6 & 1 & 10 & 273 & 274 & 6 & 3 & 10 & 1163 & 1201 & 0 & 5 & 10 & 979 & 967 \\
\hline-1 & -3 & 10 & 225 & 227 & 1 & -1 & 10 & 887 & 894 & 7 & 1 & 10 & 1065 & 1046 & 7 & 3 & 10 & 749 & 729 & 1 & 5 & 10 & 1977 & 1962 \\
\hline 1 & -3 & 10 & 168 & 197 & 2 & -1 & 10 & 1356 & 1386 & 8 & 1 & 10 & 288 & 292 & 8 & 3 & 10 & 1345 & 1357 & 2 & 5 & 10 & 399 & 383 \\
\hline 2 & -3 & 10 & 1075 & 1076 & 3 & -1 & 10 & 305 & 310 & 9 & 1 & 10 & 676 & 633 & 9 & 3 & 10 & 653 & 660 & 3 & 5 & 10 & 662 & 678 \\
\hline 4 & -3 & 10 & 854 & 858 & 5 & -1 & 10 & 600 & 618 & 10 & 1 & 10 & 407 & 388 & 10 & 3 & 10 & 749 & 749 & 4 & 5 & 10 & 478 & 480 \\
\hline 5 & -3 & 10 & 394 & 401 & 7 & -1 & 10 & 250 & 263 & -8 & 2 & 10 & 391 & 349 & 11 & 3 & 10 & 220 & 192 & 6 & 5 & 10 & 638 & 642 \\
\hline 6 & -3 & 10 & 655 & 660 & 9 & -1 & 10 & 465 & 489 & -6 & 2 & 10 & 638 & 635 & -9 & 4 & 10 & 312 & 309 & 7 & 5 & 10 & 282 & 272 \\
\hline 7 & -3 & 10 & 1034 & 1042 & -10 & 0 & 10 & 275 & 302 & -5 & 2 & 10 & 432 & 449 & -8 & 4 & 10 & 596 & 606 & 10 & 5 & 10 & 455 & 497 \\
\hline 8 & -3 & 10 & 768 & 750 & -9 & 0 & 10 & 597 & 593 & -4 & 2 & 10 & 274 & 259 & -7 & 4 & 10 & 408 & 396 & -8 & 6 & 10 & 425 & 453 \\
\hline 9 & -3 & 10 & 729 & 690 & -8 & 0 & 10 & 290 & 277 & -3 & 2 & 10 & 560 & 549 & -6 & 4 & 10 & 747 & 750 & -6 & 6 & 10 & 272 & 242 \\
\hline-10 & -2 & 10 & 343 & 374 & -7 & 0 & 10 & 312 & 330 & -2 & 2 & 10 & 867 & 868 & -5 & 4 & 10 & 613 & 645 & -5 & 6 & 10 & 838 & 821 \\
\hline-9 & -2 & 10 & 800 & 805 & -6 & 0 & 10 & 875 & 897 & -1 & 2 & 10 & 247 & 264 & -4 & 4 & 10 & 1108 & 1099 & -4 & 6 & 10 & 287 & 272 \\
\hline-7 & -2 & 10 & 910 & 907 & -5 & 0 & 10 & 551 & 541 & 0 & 2 & 10 & 343 & 329 & -3 & 4 & 10 & 237 & 268 & -3 & 6 & 10 & 1261 & 1273 \\
\hline-6 & -2 & 10 & 728 & 711 & -4 & 0 & 10 & 1729 & 1744 & 1 & 2 & 10 & 1147 & 1163 & -2 & 4 & 10 & 246 & 235 & -1 & 6 & 10 & 970 & 1012 \\
\hline-5 & -2 & 10 & 1253 & 1244 & -3 & 0 & 10 & 570 & 573 & 3 & 2 & 10 & 1948 & 1926 & -1 & 4 & 10 & 656 & 659 & 0 & 6 & 10 & 1204 & 1261 \\
\hline-4 & -2 & 10 & 459 & 463 & -2 & 0 & 10 & 1222 & 1228 & 4 & 2 & 10 & 390 & 356 & 0 & 4 & 10 & 304 & 286 & 1 & 6 & 10 & 1564 & 1601 \\
\hline-3 & -2 & 10 & 231 & 212 & -1 & 0 & 10 & 957 & 977 & 5 & 2 & 10 & 1216 & 1240 & 1 & 4 & 10 & 910 & 925 & 2 & 6 & 10 & 1448 & 1483 \\
\hline-2 & -2 & 10 & 474 & 450 & 0 & 0 & 10 & 2128 & 2115 & 6 & 2 & 10 & 975 & 997 & 2 & 4 & 10 & 468 & 466 & 3 & 6 & 10 & \pm 677 & 1674 \\
\hline
\end{tabular}




\begin{tabular}{|c|c|c|c|c|c|c|c|c|c|c|c|c|c|c|c|c|c|c|c|c|c|c|}
\hline 7 & 10 & 1719 & 1815 & 9 & 9 & 10 & 1046 & 1045 & -213 & 10 & 405 & 408 & $0-$ & -10 & 11 & 585 & 598 & 4 & -7 & 11 & 316 & 312 \\
\hline 7 & 10 & 1424 & 1454 & 10 & 9 & 10 & 318 & 281 & 013 & 10 & 283 & 297 & $1-$ & -10 & 11 & 211 & 204 & 7 & -7 & 11 & 269 & 277 \\
\hline 7 & 10 & 1284 & 1269 & 11 & 9 & 10 & 780 & 758 & 113 & 10 & 568 & 577 & & -10 & 11 & 927 & 937 & -9 & -6 & 11 & 743 & 769 \\
\hline 7 & 10 & 409 & 419 & -5 & 10 & 10 & 631 & 640 & 213 & 10 & 323 & 362 & -4 & -9 & 11 & 298 & 283 & -8 & -6 & 11 & 457 & 443 \\
\hline 7 & 10 & 823 & 791 & -4 & 10 & 10 & 506 & 505 & 313 & 10 & 837 & 866 & -3 & -9 & 11 & 485 & 496 & -7 & -6 & 11 & 685 & 676 \\
\hline 7 & 10 & 482 & 491 & -3 & 10 & 10 & 728 & 726 & 4. 13 & 10 & 412 & 396 & -2 & -9 & 11 & 390 & 385 & -6 & -6 & 11 & 1090 & 1078 \\
\hline 7 & 10 & 909 & 897 & 0 & 10 & 10 & 392 & 434 & 513 & 10 & 612 & 582 & -1 & -9 & 11 & 428 & 464 & -5 & -6 & 11 & 4.58 & 458 \\
\hline 7 & 10 & 496 & 516 & 1 & 10 & 10 & 284 & 325 & 713 & 10 & 695 & 668 & 0 & -9 & 11 & 614 & 630 & -4 & -6 & 11 & 1643 & 1669 \\
\hline 7 & 10 & 538 & 565 & 2 & 10 & 10 & 462 & 499 & 813 & 10 & 323 & 307 & 2 & -9 & 11 & 1039 & 1056 & -3 & -6 & 11 & 459 & 452 \\
\hline 7 & 10 & 295 & 304 & 3 & 10 & 10 & 191 & 166 & 913 & 10 & 676 & 663 & 4 & -9 & 11 & 675 & 690 & -2 & -6 & 11 & 960 & 958 \\
\hline 8 & 10 & 323 & 353 & 7 & 10 & 10 & 383 & 382 & 314 & 10 & 558 & 574 & 5 & -9 & 11 & 247 & 334 & -1 & -6 & 11 & 539 & 594 \\
\hline 8 & 10 & 381 & 368 & 9 & 10 & 10 & 809 & 797 & 514 & 10 & 590 & 587 & -9 & -8 & 11 & 588 & 610 & 0 & -6 & 11 & 1074 & 1065 \\
\hline 8 & 10 & 294 & 279 & 11 & 10 & 10 & 519 & 502 & 714 & 10 & 320 & 266 & -7 & $-B$ & 11 & 428 & 392 & 1 & -6 & 11 & 621 & 649 \\
\hline 8 & 10 & 287 & 270 & -4 & 11 & 10 & 656 & 654 & 814 & 10 & 538 & 511 & -6 & -8 & 11 & 408 & 402 & 2 & -6 & 11 & 990 & 1039 \\
\hline 8 & 10 & 964 & 1024 & -3 & 11 & 10 & 502 & 488 & 914 & 10 & 403 & 355 & -5 & -8 & 11 & 393 & 423 & -10 & -5 & 11 & 245 & 238 \\
\hline 8 & 10 & 278 & 296 & -2 & 11 & 10 & 538 & 538 & 115 & 10 & 271 & 267 & -4 & -8 & 11 & 295 & 316 & -9 & -5 & 11 & 367 & 387 \\
\hline 8 & 10 & 1073 & 1107 & 0 & 11 & 10 & 562 & 572 & 515 & 10 & 329 & 313 & -2 & -8 & 11 & 196 & 106 & -8 & -5 & 11 & 297 & 239 \\
\hline 8 & 10 & 543 & 530 & 1 & 11 & 10 & 561 & 576 & $-5-12$ & 11 & 972 & 977 & -1 & -8 & 11 & 358 & 361 & -7 & -5 & 11 & 300 & 273 \\
\hline 8 & 10 & 757 & 744 & 2 & 11 & 10 & 650 & 666 & $-4-12$ & 11 & 515 & 466 & 1 & -8 & 11 & 295 & 311 & -6 & -5 & 11 & 717 & 725 \\
\hline 8 & 10 & 568 & 598 & 3 & 11 & 10 & 436 & 421 & $-3-12$ & 11 & 631 & 633 & 2 & -8 & 11 & 584 & 579 & -4 & -5 & 11 & 1770 & 1772 \\
\hline 8 & 10 & 828 & 840 & 4 & 11 & 10 & 323 & 299 & $-1-12$ & 11 & 446 & 440 & 4 & -8 & 11 & 713 & 695 & -3 & -5 & 11 & 259 & 243 \\
\hline 8 & 10 & 629 & 613 & 5 & 11 & 10 & 296 & 289 & $-7-1.1$ & 11 & 411 & 405 & 6 & -8 & 11 & 216 & 251 & -2 & -5 & 11 & 1352 & 1372 \\
\hline 8 & 10 & 681 & 664 & 7 & 11 & 10 & 540 & 546 & $-5-11$ & 11 & 939 & 9.34 & -9 & -7 & 11 & 815 & 804 & -1 & -5 & 11 & 343 & 380 \\
\hline 9 & 10 & 302 & 298 & 8 & 11 & 10 & 199 & 161 & $-3-11$ & 11 & 901 & 937 & -8 & -7 & 1.1 & 230 & 233 & 0 & -5 & 11 & 581 & 610 \\
\hline 9 & 10 & 752 & 706 & 11 & 11 & 10 & 309 & 249 & $-1-11$ & 11 & 402 & 400 & -7 & -7 & 11 & 523 & 500 & 1 & -5 & 11 & 1355 & 1376 \\
\hline 9 & 10 & 449 & 417 & -2 & 12 & 10 & 517 & 532 & $0-11$ & 11 & 546 & 539 & -6 & -7 & 11 & 817 & 817 & 2 & -5 & 11 & 877 & 879 \\
\hline
\end{tabular}

Observed and calculated structure factors: $\quad\left[\mathrm{OS}(\mathrm{CO})_{2} \mathrm{PPH}_{3}\right]_{2}$ (MU-I) (MU-PPH $\left.{ }_{2}\right)$

Page 28

\section{h $\mathrm{k} I 10 \mathrm{FO} 10 \mathrm{FC}$}

$\begin{array}{lllll}3 & -5 & 11 & 940 & 972\end{array}$

$\begin{array}{lllll}4 & -5 & 11 & 192 & 226\end{array}$

$\begin{array}{lllll}5 & -5 & 11 & 566 & 580\end{array}$

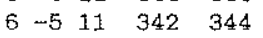

$\begin{array}{lllll}7 & -5 & 11 & 589 & 579\end{array}$

$\begin{array}{lllll}-7 & -4 & 11 & 395 & 388\end{array}$

$\begin{array}{lllll}-6 & -4 & 11 & 209 & 196\end{array}$

$\begin{array}{lllll}-5 & -4 & 11 & 554 & 542\end{array}$

$\begin{array}{lllll}-4 & -4 & 11 & 941 & 918\end{array}$

$\begin{array}{lllll}-3 & -4 & 11 & 926 & 909\end{array}$

$\begin{array}{llllll}-2 & -4 & 11 & 1429 & 1433\end{array}$

$\begin{array}{lllll}-1 & -4 & 11 & 447 & 453\end{array}$

$\begin{array}{lllll}0 & -4 & 11 & 303 & 300\end{array}$

$\begin{array}{lllll}1 & -4 & 11 & 1350 & 1369\end{array}$

$\begin{array}{llllll}3 & -4 & 11 & 1767 & 1742\end{array}$

$\begin{array}{lllll}5 & -4 & 11 & 1004 & 996\end{array}$

$\begin{array}{lllll}6 & -4 & 11 & 507 & 500\end{array}$

$\begin{array}{llllll}7 & -4 & 11 & 854 & 853\end{array}$

$\begin{array}{lllll}8 & -4 & 11 & 539 & 534\end{array}$

$\begin{array}{lllll}9 & -4 & 11 & 543 & 525\end{array}$

$\begin{array}{lllll}-10 & -3 & 11 & 433 & 436\end{array}$

$\begin{array}{llllll}-9 & -3 & 11 & 224 & 271\end{array}$

$\begin{array}{llllll}-8 & -3 & 11 & 220 & 258\end{array}$

$\begin{array}{llllll}-7 & -3 & 11 & 519 & 508\end{array}$

$\begin{array}{lllll}-6 & -3 & 11 & 625 & 612\end{array}$

$\begin{array}{llllll}-4 & -3 & 11 & 308 & 302\end{array}$

$\begin{array}{lllll}-3 & -3 & 11 & 939 & 937\end{array}$

$\begin{array}{lllll}-2 & -3 & 11 & 720 & 749\end{array}$

$\begin{array}{lllll}-1 & -3 & 11 & 388 & 443\end{array}$

$\begin{array}{lllll}0 & -3 & 11 & 240 & 212\end{array}$

$\begin{array}{lllll}1 & -3 & 11 & 855 & 857\end{array}$

$\begin{array}{lllll}2 & -3 & 11 & 430 & 428\end{array}$

$\begin{array}{llllll}3 & -3 & 11 & 1624 & 1631\end{array}$

$\begin{array}{lllll}4 & -3 & 11 & 253 & 248\end{array}$ h k l 10FO 10FC

$\begin{array}{llllll}5 & -3 & 11 & 1027 & 1024\end{array}$

$\begin{array}{lllll}6 & -3 & 11 & 814 & 834\end{array}$

$\begin{array}{lllll}7 & -3 & 11 & 766 & 767\end{array}$

$\begin{array}{lllll}8 & -3 & 11 & 897 & 838\end{array}$

$\begin{array}{lllll}9 & -3 & 11 & 630 & 598\end{array}$

$\begin{array}{lllll}-10 & -2 & 11 & 916 & 855\end{array}$

$\begin{array}{lllll}-9 & -2 & 11 & 409 & 410\end{array}$

$\begin{array}{lllll}-8 & -2 & 11 & 565 & 573\end{array}$

$\begin{array}{lllll}-7 & -2 & 11 & 236 & 201\end{array}$

$\begin{array}{lllll}-6 & -2 & 11 & 403 & 382\end{array}$

$\begin{array}{lllll}-5 & -2 & 11 & 751 & 730\end{array}$

$\begin{array}{lllll}-4 & -2 & 11 & 792 & 815\end{array}$

$\begin{array}{lllll}-1 & -2 & 11 & 189 & 212\end{array}$

$\begin{array}{lllll}0 & -2 & 11 & 367 & 353\end{array}$

$\begin{array}{lllll}2 & -2 & 11 & 264 & 297\end{array}$

$\begin{array}{lllll}3 & -2 & 11 & 952 & 989\end{array}$

$\begin{array}{lllll}4 & -2 & 11 & 354 & 357\end{array}$

$\begin{array}{lllll}5 & -2 & 11 & 743 & 734\end{array}$

$\begin{array}{lllll}6 & -2 & 11 & 577 & 570\end{array}$

$\begin{array}{lllll}8 & -2 & 11 & 947 & 917\end{array}$

$\begin{array}{lllll}10 & -2 & 11 & 692 & 710\end{array}$

$\begin{array}{lllll}-10 & -1 & 11 & 909 & 906\end{array}$

$\begin{array}{llllll}-8 & -1 & 11 & 850 & 841\end{array}$

$\begin{array}{llllll}-7 & -1 & 11 & 248 & 244\end{array}$

$\begin{array}{lllll}-5 & -1 & 11 & 448 & 441\end{array}$

$\begin{array}{lllll}-5 & -1 & 11 & 1240 & 1258\end{array}$

$\begin{array}{lllll}-4 & -1 & 11 & 681 & 654\end{array}$

$\begin{array}{llllll}-3 & -1 & 11 & 1497 & 1469\end{array}$

$\begin{array}{llllll}-1 & -1 & 11 & 1066 & 1082\end{array}$

$\begin{array}{lllll}0 & -1 & 11 & 734 & 744\end{array}$

$\begin{array}{lllll}1 & -1 & 11 & 1002 & 1020\end{array}$

$\begin{array}{lllll}2 & -1 & 11 & 240 & 229\end{array}$

$\begin{array}{lllll}3 & -1 & 11 & 175 & 45\end{array}$

$4-1 \quad 11250 \quad 253$ h $\mathrm{k}$ I 1OFO 10F'C

$\begin{array}{lllll}5 & -1 & 11 & 563 & 564\end{array}$

$\begin{array}{lllll}8 & -1 & 11 & 470 & 446\end{array}$

$\begin{array}{lllll}9 & -1 & 11 & 352 & 316\end{array}$

$\begin{array}{llllll}10 & -1 & 11 & 585 & 565\end{array}$

$\begin{array}{lllll}-9 & 0 & 11 & 487 & 473\end{array}$

$\begin{array}{lllll}-8 & 0 & 11 & 810 & 830\end{array}$

$\begin{array}{lllll}-7 & 0 & 11 & 368 & 368\end{array}$

$\begin{array}{lllll}-6 & 0 & 11 & 279 & 283\end{array}$

$\begin{array}{lllll}-5 & 0 & 11 & 1208 & 1194\end{array}$

$\begin{array}{lllll}-3 & 0 & 11 & 2053 & 2034\end{array}$

$\begin{array}{lllll}-2 & 0 & 11 & 173 & 136\end{array}$

$\begin{array}{llllll}-1 & 0 & 11 & 1383 & 1380\end{array}$

o $\quad 0 \quad 1111041061$.

$\begin{array}{lllll}1 & 0 & 11 & 2022 & 2057\end{array}$

$\begin{array}{lllll}2 & 0 & 11 & 895 & 899\end{array}$

$\begin{array}{lllll}3 & 0 & 11 & 1127 & 1143\end{array}$

$\begin{array}{lllll}4 & 0 & 11 & 218 & 204\end{array}$

$\begin{array}{lllll}6 & 0 & 11 & 657 & 686\end{array}$

$\begin{array}{lllll}7 & 0 & 11 & 411 & 392\end{array}$

$\begin{array}{lllll}8 & 0 & 11 & 355 & 342\end{array}$

$\begin{array}{lllll}9 & 0 & 11 & 273 & 275\end{array}$

$\begin{array}{lllll}-9 & 1 & 11 & 409 & 386\end{array}$

$\begin{array}{lllll}-8 & 1 & 11 & 489 & 479\end{array}$

$\begin{array}{lllll}-7 & 1 & 11 & 210 & 275\end{array}$

$\begin{array}{lllll}-5 & 1 & 11 & 1003 & 994\end{array}$

$\begin{array}{lllll}-4 & 1 & 11 & 491 & 505\end{array}$

$\begin{array}{lllll}-3 & 1 & 11 & 1903 & 1921\end{array}$

$\begin{array}{lllll}-2 & 1 & 11 & 195 & 167\end{array}$

$\begin{array}{llllll}-1 & 1 & 11 & 1329 & 1333\end{array}$

o 111111901166

$111111152 \quad 1139$

$\begin{array}{lllll}2 & 1 & 11 & 2015 & 1997\end{array}$

$\begin{array}{llllll}3 & 1 & 11 & 1044 & 1032\end{array}$

411110401016 h $\mathrm{k} \perp$ 10Fo 10FC

$\begin{array}{lllll}5 & 1 & 11 & 278 & 303\end{array}$

$\begin{array}{lllll}6 & 1 & 11 & 614 & 602\end{array}$

$\begin{array}{lllll}7 & 1 & 1.1 & 656 & 665\end{array}$

$\begin{array}{lllll}8 & 1 & 11 & 946 & 918\end{array}$

$\begin{array}{lllll}9 & 1 & 11 & 233 & 180\end{array}$

$\begin{array}{lllll}10 & 1 & 11 & 316 & 314\end{array}$

$\begin{array}{lllll}-4 & 2 & 11 & 789 & 741\end{array}$

$\begin{array}{lllll}-3 & 2 & 11 & 1161 & 1170\end{array}$

$\begin{array}{lllll}-2 & 2 & 11 & 478 & 454\end{array}$

$\begin{array}{lllll}-1 & 2 & 11 & 1307 & 1326\end{array}$

$\begin{array}{lllll}0 & 2 & 11 & 717 & 741\end{array}$

$\begin{array}{lllll}1 & 2 & 11 & 251 & 240\end{array}$

$\begin{array}{lllll}2 & 2 & 11 & 1757 & 1782\end{array}$

$\begin{array}{lllll}3 & 2 & 11 & 438 & 438\end{array}$

$\begin{array}{lllll}4 & 2 & 11 & 1784 & 1792\end{array}$

$\begin{array}{lllll}6 & 2 & 11 & 842 & 835\end{array}$

$722 \quad 111071 \quad 1048$

$\begin{array}{llllll}8 & 2 & 11 & 809 & 845\end{array}$

$\begin{array}{lllll}9 & 2 & 11 & 813 & 782\end{array}$

$\begin{array}{lllll}10 & 2 & 11 & 448 & 420\end{array}$

$\begin{array}{lllll}11 & 2 & 11 & 441 & 420\end{array}$

$\begin{array}{llllll}-9 & 3 & 11 & 464 & 445\end{array}$

$\begin{array}{lllll}-7 & 3 & 11 & 413 & 422\end{array}$

$\begin{array}{lllll}-6 & 3 & 11 & 387 & 385\end{array}$

$\begin{array}{lllll}-5 & 3 & 11 & 828 & 841\end{array}$

$\begin{array}{lllll}-4 & 3 & 11 & 409 & 419\end{array}$

$\begin{array}{lllll}-2 & 3 & 11 & 656 & 652\end{array}$

$\begin{array}{lllll}-1 & 3 & 11 & 697 & 701\end{array}$

$\begin{array}{lllll}0 & 3 & 11 & 168 & 121\end{array}$

$\begin{array}{lllll}1 & 3 & 11 & 174 & 158\end{array}$

$\begin{array}{lllll}2 & 3 & 11 & 819 & 815\end{array}$

$\begin{array}{lllll}3 & 3 & 11 & 467 & 497\end{array}$

$\begin{array}{lllll}4 & 3 & 11 & 1995 & 1969\end{array}$

$\begin{array}{lllll}5 & 3 & 11 & 454 & 436\end{array}$ $\mathrm{k} \mathrm{k} \perp 1$ 10FO 1OFC

$\begin{array}{lllll}6 & 3 & 11 & 714 & 749\end{array}$

$\begin{array}{lllll}7 & 3 & 11 & 1113 & 1119\end{array}$

$8 \quad 311 \quad 604 \quad 554$

$\begin{array}{lllll}9 & 3 & 11 & 1011 & 1011\end{array}$

$\begin{array}{lllll}10 & 3 & 11 & 380 & 381\end{array}$

$\begin{array}{lllll}11 & 3 & 11 & 651 & 665\end{array}$

$\begin{array}{llllll}-7 & 4 & 11 & 445 & 430\end{array}$

$\begin{array}{lllll}-6 & 4 & 11 & 736 & 695\end{array}$

$\begin{array}{lllll}-5 & 4 & 11 & 700 & 723\end{array}$

$\begin{array}{lllll}-4 & 4 & 11 & 623 & 628\end{array}$

$\begin{array}{llllll}-3 & 4 & 11 & 746 & 782\end{array}$

$-2 \quad 4 \quad 11 \quad 220 \quad 186$

$\begin{array}{lllll}-1 & 4 & 11 & 234 & 248\end{array}$

$\begin{array}{lllll}1 & 4 & 11 & 408 & 410\end{array}$

$\begin{array}{lllll}2 & 4 & 11 & 508 & 475\end{array}$

$\begin{array}{lllll}3 & 4 & 11 & 628 & 651\end{array}$

$4411 \quad 870 \quad 919$

$5 \quad 4 \quad 11629 \quad 648$

$\begin{array}{lllll}6 & 4 & 11 & 616 & 593\end{array}$

$7 \quad 4 \quad 11 \quad 959 \quad 1015$

$94 \quad 4 \quad 11 \quad 927 \quad 936$

$10411 \quad 319 \quad 305$

$\begin{array}{lllll}11 & 4 & 11 & 704 & 688\end{array}$

$\begin{array}{lllll}-7 & 5 & 11 & 488 & 493\end{array}$

$\begin{array}{lllll}-6 & 5 & 11 & 648 & 663\end{array}$

$\begin{array}{lllll}-5 & 5 & 11 & 293 & 245\end{array}$

$\begin{array}{llllll}-4 & 5 & 11 & 1184 & 1163\end{array}$

$\begin{array}{lllll}-3 & 5 & 11 & 554 & 565\end{array}$

$\begin{array}{lllll}-2 & 5 & 11 & 795 & 792\end{array}$

$\begin{array}{lllll}\text { O } & 5 & 11 & 487 & 490\end{array}$

$\begin{array}{lllll}1 & 5 & 11 & 922 & 938\end{array}$

$\begin{array}{lllll}2 & 5 & 11 & 1039 & 1053\end{array}$

$\begin{array}{lllll}4 & 5 & 11 & 246 & 232\end{array}$

$\begin{array}{lllll}6 & 5 & 11 & 212 & 224\end{array}$ 
$\begin{array}{llllll}1 & 6 & 11 & 1434 & 1454\end{array}$

$\begin{array}{lllll}6 & 11 & 1152 & 1190\end{array}$

$\begin{array}{lllll}3 & 6 & 11 & 675 & 664\end{array}$

$\begin{array}{lllll}5 & 6 & 11 & 455 & 464\end{array}$

$\begin{array}{llllll}6 & 6 & 11 & 448 & 450\end{array}$

$\begin{array}{lllll}7 & 6 & 11 & 684 & 686\end{array}$

$\begin{array}{lllll}10 & 6 & 11 & 302 & 271\end{array}$

$\begin{array}{lllll}11 & 6 & 11 & 272 & 202\end{array}$

$\begin{array}{lllll}-7 & 7 & 11 & 285 & 288\end{array}$

$\begin{array}{lllll}-6 & 7 & 11 & 221 & 203\end{array}$

$-4 \quad 7 \quad 11586 \quad 573$

$\begin{array}{lllll}-3 & 7 & 11 & 320 & 347\end{array}$

$\begin{array}{llllll}-2 & 7 & 11 & 1277 & 1280\end{array}$

$\begin{array}{lllll}-1 & 7 & 11 & 452 & 449\end{array}$

$\begin{array}{lllll}0 & 7 & 11 & 1007 & 1037\end{array}$

$\begin{array}{llllll}1 & 7 & 11 & 1424 & 1469\end{array}$

$\begin{array}{llllll}2 & 7 & 11 & 1166 & 1230\end{array}$

$\begin{array}{llllll}3 & 7 & 11 & 1736 & 1752\end{array}$

$\begin{array}{lllll}4 & 7 & 11 & 977 & 962\end{array}$

$\begin{array}{lllll}5 & 7 & 11 & 602 & 621\end{array}$

$\begin{array}{lllll}7 & 7 & 11 & 758 & 782\end{array}$

$\begin{array}{lllll}8 & 7 & 11 & 652 & 670\end{array}$

$\begin{array}{lllll}9 & 7 & 11 & 686 & 685\end{array}$

$\begin{array}{lllll}10 & 7 & 11 & 338 & 343\end{array}$

$\begin{array}{lllll}-3 & 8 & 11 & 433 & 407\end{array}$ $\begin{array}{lllll}8 & 8 & 11 & 752 & 767\end{array}$

$\begin{array}{lllll}9 & 8 & 11 & 611 & 612\end{array}$

$\begin{array}{lllll}10 & 8 & 11 & 742 & 759\end{array}$

$\begin{array}{lllll}11 & 8 & 11 & 328 & 284\end{array}$

$\begin{array}{lllll}-5 & 9 & 11 & 307 & 299\end{array}$

$\begin{array}{lllll}-4 & 9 & 11 & 361 & 362\end{array}$

$\begin{array}{lllll}0 & 9 & 11 & 350 & 354\end{array}$

$\begin{array}{lllll}2 & 9 & 11 & 473 & 504\end{array}$

$\begin{array}{lllll}3 & 9 & 11 & 754 & 765\end{array}$

$4911685 \quad 706$

$\begin{array}{lllll}5 & 9 & 11 & 776 & 799\end{array}$

$\begin{array}{lllll}6 & 9 & 11 & 247 & 237\end{array}$

$\begin{array}{lllll}7 & 9 & 11 & 273 & 282\end{array}$

$\begin{array}{lllll}8 & 9 & 11 & 880 & 873\end{array}$

$\begin{array}{lllll}9 & 9 & 11 & 364 & 333\end{array}$

$\begin{array}{lllll}10 & 9 & 11 & 984 & 963\end{array}$

$\begin{array}{lllll}-5 & 10 & 11 & 461 & 465\end{array}$

$\begin{array}{lllll}-4 & 10 & 11 & 519 & 507\end{array}$

$\begin{array}{lllll}-3 & 10 & 11 & 376 & 372\end{array}$

$\begin{array}{lllll}-2 & 10 & 11 & 360 & 336\end{array}$

$\begin{array}{lllll}0 & 10 & 11 & 315 & 351\end{array}$

$11011 \quad 580 \quad 605$

$\begin{array}{lllll}3 & 10 & 11 & 197 & 193\end{array}$

$\begin{array}{lllll}4 & 10 & 11 & 601 & 599\end{array}$

$\begin{array}{lllll}5 & 10 & 11 & 539 & 505\end{array}$

$\begin{array}{llllll}6 & 10 & 11 & 541 & 567\end{array}$ $\begin{array}{lllll}11 & 11 & 438 & 449\end{array}$

$\begin{array}{lllll}2 & 11 & 11 & 451 & 464\end{array}$

$\begin{array}{lllll}9 & 11 & 11 & 249 & 248\end{array}$

$\begin{array}{lllll}10 & 11 & 11 & 433 & 413\end{array}$

$\begin{array}{lllll}-3 & 12 & 11 & 661 & 629\end{array}$

$\begin{array}{lllll}-1 & 12 & 11 & 519 & 573\end{array}$

$\begin{array}{lllll}0 & 12 & 11 & 258 & 190\end{array}$

$\begin{array}{llllll}1 & 12 & 11 & 301 & 334\end{array}$

$\begin{array}{lllll}2 & 12 & 11 & 811 & 853\end{array}$

$\begin{array}{lllll}3 & 12 & 11 & 521 & 529\end{array}$

$\begin{array}{lllll}4 & 12 & 11 & 466 & 486\end{array}$

$\begin{array}{lllll}5 & 12 & 11 & 197 & 157\end{array}$

$\begin{array}{lllll}6 & 12 & 11 & 424 & 404\end{array}$

$\begin{array}{llllll}8 & 12 & 11 & 447 & 400\end{array}$

$\begin{array}{llllll}-1 & 13 & 11 & 513 & 504\end{array}$

$\begin{array}{lllll}0 & 13 & 11 & 284 & 338\end{array}$

$\begin{array}{lllll}1 & 13 & 11 & 220 & 239\end{array}$

$\begin{array}{lllll}2 & 13 & 11 & 844 & 854\end{array}$

$\begin{array}{lllll}3 & 13 & 11 & 319 & 312\end{array}$

$\begin{array}{lllll}4 & 13 & 11 & 876 & 847\end{array}$

$\begin{array}{llllll}6 & 13 & 11 & 660 & 643\end{array}$

$\begin{array}{lllll}7 & 13 & 11 & 237 & 265\end{array}$

$\begin{array}{lllll}8 & 13 & 11 & 520 & 523\end{array}$

$\begin{array}{lllll}9 & 13 & 11 & 357 & 352\end{array}$

$\begin{array}{lllll}2 & 14 & 11 & 555 & 560\end{array}$ $\begin{array}{llll}-2-12 & 12 & 363 & 343\end{array}$

$\begin{array}{rrrr}-4-11 & 12 & 1030 & 1048\end{array}$

$\begin{array}{llll}-2-11 & 12 & 651 & 632\end{array}$

$\begin{array}{llll}0-11 & 12 & 305 & 307\end{array}$

1-11 $12 \quad 492 \quad 524$

$\begin{array}{llll}-6-10 & 12 & 358 & 362\end{array}$

$\begin{array}{llll}-5-10 & 12 & 340 & 334\end{array}$

$\begin{array}{llll}-4-10 & 12 & 844 & 888\end{array}$

$\begin{array}{llll}-3-10 & 12 & 459 & 479\end{array}$

$\begin{array}{llll}-2-10 & 12 & 773 & 757\end{array}$

$\begin{array}{llll}-1-10 & 12 & 282 & 256\end{array}$

$\begin{array}{lllll}1-10 & 12 & 699 & 696\end{array}$

$\begin{array}{llll}3-10 & 12 & 862 & 849\end{array}$

$\begin{array}{lllll}-8 & -9 & 12 & 260 & 184\end{array}$

$\begin{array}{lllll}-5 & -9 & 12 & 322 & 297\end{array}$

$\begin{array}{lllll}-3 & -9 & 12 & 789 & 787\end{array}$

$\begin{array}{lllll}-2 & -9 & 12 & 754 & 758\end{array}$

$\begin{array}{lllll}-1 & -9 & 12 & 685 & 672\end{array}$

$\begin{array}{lllll}1 & -9 & 12 & 861 & 833\end{array}$

$\begin{array}{lllll}2 & -9 & 12 & 232 & 199\end{array}$

$\begin{array}{lllll}3 & -9 & 12 & 998 & 992\end{array}$

$\begin{array}{lllll}4 & -9 & 12 & 328 & 291\end{array}$

$\begin{array}{lllll}-7 & -8 & 12 & 215 & 186\end{array}$

$\begin{array}{lllll}-6 & -8 & 12 & 265 & 216\end{array}$

$\begin{array}{lllll}-5 & -8 & 12 & 211 & 200\end{array}$ $\begin{array}{lllll}5 & -8 & 12 & 619 & 601\end{array}$

$\begin{array}{lllll}-7 & -7 & 12 & 263 & 293\end{array}$

$\begin{array}{lllll}-5 & -7 & 12 & 890 & 909\end{array}$

$\begin{array}{lllll}-4 & -7 & 12 & 309 & 309\end{array}$

$\begin{array}{lllll}-3 & -7 & 12 & 558 & 564\end{array}$

$\begin{array}{lllll}2 & -7 & 12 & 288 & 346\end{array}$

$\begin{array}{lllll}3 & -7 & 12 & 271 & 277\end{array}$

$\begin{array}{lllll}4 & -7 & 12 & 373 & 385\end{array}$

$\begin{array}{lllll}5 & -7 & 12 & 520 & 537\end{array}$

$\begin{array}{lllll}-9 & -6 & 12 & 399 & 428\end{array}$

$\begin{array}{lllll}-8 & -6 & 12 & 798 & 791\end{array}$

$\begin{array}{lllll}-7 & -6 & 12 & 447 & 445\end{array}$

$\begin{array}{lllll}-6 & -6 & 12 & 231 & 221\end{array}$

$\begin{array}{lllll}-5 & -6 & 12 & 1316 & 1329\end{array}$

$\begin{array}{lllll}-3 & -6 & 12 & 1261 & 1282\end{array}$

$\begin{array}{lllll}-1 & -6 & 12 & 778 & 791\end{array}$

$\begin{array}{lllll}0 & -6 & 12 & 556 & 565\end{array}$

$\begin{array}{lllll}1 & -5 & 12 & 631 & 638\end{array}$

$\begin{array}{lllll}2 & -6 & 12 & 344 & 350\end{array}$

$\begin{array}{lllll}3 & -6 & 12 & 476 & 449\end{array}$

$\begin{array}{lllll}4 & -6 & 12 & 342 & 303\end{array}$

$\begin{array}{lllll}5 & -6 & 12 & 281 & 285\end{array}$

$\begin{array}{lllll}-9 & -5 & 1.2 & 549 & 563\end{array}$

$\begin{array}{lllll}-8 & -5 & 12 & 522 & 505\end{array}$

$\begin{array}{lllll}-7 & -5 & 12 & 707 & 706\end{array}$

Observed and calculated structure factors: [OS(CO) $\left.{ }_{2} \mathrm{PPH}_{3}\right]_{2}$ (MU-I) (MU-PPH ${ }_{2}$ )

Page 30

$\begin{array}{rrrrrrrrrr}\mathrm{h} & \mathrm{k} & 1 & 1 \text { IOFO } & 1 \text { OFc } & \mathrm{h} & \mathrm{k} & \mathrm{l} & 1 \text { OFO } & 1 \text { IOFC } \\ -5 & -5 & 12 & 1378 & 1405 & 6 & -3 & 12 & 860 & 858 \\ -4 & -5 & 12 & 304 & 286 & 7 & -3 & 12 & 775 & 776 \\ -3 & -5 & 12 & 1750 & 1790 & 8 & -3 & 12 & 510 & 538 \\ -1 & -5 & 12 & 1031 & 1037 & 9 & -3 & 12 & 646 & 639 \\ 0 & -5 & 12 & 772 & 765 & -9 & -2 & 12 & 603 & 602 \\ 1 & -5 & 12 & 342 & 368 & -7 & -2 & 12 & 282 & 258 \\ 2 & -5 & 12 & 1161 & 1158 & -6 & -2 & 12 & 455 & 446 \\ 3 & -5 & 12 & 612 & 591 & -5 & -2 & 12 & 273 & 307 \\ 4 & -5 & 12 & 318 & 308 & -3 & -2 & 12 & 536 & 561 \\ 6 & -5 & 12 & 203 & 187 & -2 & -2 & 12 & 795 & 810 \\ 7 & -5 & 12 & 249 & 266 & -1 & -2 & 12 & 525 & 542 \\ -9 & -4 & 12 & 248 & 221 & 0 & -2 & 12 & 461 & 514 \\ -5 & -4 & 12 & 749 & 719 & 2 & -2 & 12 & 766 & 779 \\ -4 & -4 & 12 & 695 & 698 & 3 & -2 & 12 & 925 & 928 \\ -3 & -4 & 12 & 1479 & 1541 & 4 & -2 & 12 & 1232 & 1223 \\ -2 & -4 & 12 & 629 & 647 & 5 & -2 & 12 & 784 & 796 \\ -1 & -4 & 12 & 1261 & 1282 & 6 & -2 & 12 & 681 & 693 \\ 0 & -4 & 12 & 680 & 645 & 7 & -2 & 12 & 801 & 812 \\ 2 & -4 & 12 & 1608 & 1637 & 8 & -2 & 12 & 264 & 223 \\ 4 & -4 & 12 & 1364 & 1388 & 9 & -2 & 12 & 945 & 877 \\ 6 & -4 & 12 & 577 & 596 & -9 & -1 & 12 & 896 & 923 \\ 7 & -4 & 12 & 541 & 539 & -7 & -1 & 12 & 561 & 545 \\ 8 & -4 & 12 & 284 & 317 & -6 & -1 & 12 & 461 & 447 \\ -9 & -3 & 12 & 241 & 253 & -4 & -1 & 12 & 1183 & 1186 \\ -4 & -3 & 12 & 747 & 767 & -3 & -1 & 12 & 631 & 617 \\ -3 & -3 & 12 & 392 & 374 & -2 & -1 & 12 & 695 & 692 \\ -2 & -3 & 12 & 1348 & 1394 & 0 & -1 & 12 & 274 & 285 \\ -1 & -3 & 12 & 926 & 935 & 1 & -1 & 12 & 690 & 717 \\ 0 & -3 & 12 & 489 & 498 & 2 & -1 & 12 & 221 & 203 \\ 1 & -3 & 12 & 289 & 270 & 3 & -1 & 12 & 423 & 421 \\ 2 & -3 & 12 & 1213 & 1244 & 4 & -1 & 12 & 541 & 542 \\ 3 & -3 & 12 & 583 & 567 & 5 & -1 & 12 & 606 & 595 \\ 4 & -3 & 12 & 1621 & 1603 & 6 & -1 & 12 & 446 & 434 \\ 5 & -3 & 12 & 585 & 577 & 7 & -1 & 12 & 495 & 473\end{array}$

$\mathrm{h} k 1$ 10FO 10FC

$\begin{array}{lllll}8 & -1 & 12 & 330 & 298\end{array}$

$\begin{array}{ccccc}9 & -1 & 12 & 737 & 746\end{array}$

$\begin{array}{llllll}-9 & 0 & 12 & 794 & 767\end{array}$

$\begin{array}{llllll}-8 & 0 & 12 & 484 & 449\end{array}$

$\begin{array}{lllll}-7 & 0 & 12 & 575 & 588\end{array}$

$\begin{array}{lllll}-6 & 0 & 12 & 589 & 570\end{array}$

$\begin{array}{lllll}-5 & 0 & 12 & 441 & 423\end{array}$

$\begin{array}{lllll}-4 & 0 & 12 & 1586 & 1602\end{array}$

$\begin{array}{lllll}-3 & 0 & 12 & 213 & 209\end{array}$

$\begin{array}{llllll}-2 & 0 & 12 & 1709 & 1744\end{array}$

$\begin{array}{lllll}-1 & 0 & 12 & 251 & 276\end{array}$

$\begin{array}{lllll}0 & 0 & 12 & 946 & 921\end{array}$

$\begin{array}{lllll}1 & 0 & 12 & 875 & 855\end{array}$

$\begin{array}{llllll}2 & 0 & 12 & 1086 & 11.20\end{array}$

$\begin{array}{lllll}3 & 0 & 12 & 173 & 139\end{array}$

$\begin{array}{lllll}4 & 0 & 12 & 436 & 436\end{array}$

$\begin{array}{lllll}6 & 0 & 12 & 449 & 461\end{array}$

$\begin{array}{lllll}8 & 0 & 12 & 288 & 251\end{array}$

$\begin{array}{lllll}10 & 0 & 12 & 408 & 421\end{array}$

$\begin{array}{lllll}-9 & 1 & 12 & 274 & 267\end{array}$

$\begin{array}{lllll}-8 & 1 & 12 & 771 & 768\end{array}$

$\begin{array}{lllll}-7 & 1 & 12 & 562 & 545\end{array}$

$\begin{array}{lllll}-6 & 1 & 12 & 658 & 662\end{array}$

$\begin{array}{lllll}-4 & 1 & 12 & 1390 & 1412\end{array}$

$\begin{array}{llllll}-3 & 1 & 12 & 688 & 715\end{array}$

$\begin{array}{llllll}-2 & 1 & 12 & 1850 & 1856\end{array}$

$\begin{array}{lllll}-1 & 1 & 12 & 357 & 411\end{array}$

$\begin{array}{lllll}0 & 1 & 12 & 1006 & 1023\end{array}$

$\begin{array}{llllll}1 & 1 & 12 & 1619 & 1647\end{array}$

$\begin{array}{llllll}2 & 1 & 12 & 1145 & 1157\end{array}$

$\begin{array}{llllll}3 & 1 & 12 & 1447 & 1445\end{array}$

$\begin{array}{lllll}4 & 1 & 12 & 592 & 617\end{array}$

$\begin{array}{lllll}5 & 1 & 12 & 309 & 282\end{array}$

$\begin{array}{lllll}6 & 1 & 12 & 207 & 235\end{array}$ h $k \perp 1$ 10FO 10FC

$\begin{array}{lllll}7 & 1 & 12 & 343 & 340\end{array}$

$\begin{array}{lllll}8 & 1 & 12 & 295 & 262\end{array}$

$\begin{array}{lllll}9 & 1 & 12 & 395 & 386\end{array}$

$\begin{array}{lllll}-8 & 2 & 12 & 445 & 434\end{array}$

$\begin{array}{lllll}-7 & 2 & 12 & 268 & 255\end{array}$

$\begin{array}{lllll}-6 & 2 & 1.2 & 427 & 394\end{array}$

$\begin{array}{lllll}-4 & 2 & 12 & 781 & 789\end{array}$

$\begin{array}{lllll}-3 & 2 & 12 & 912 & 893\end{array}$

$\begin{array}{lllll}-2 & 2 & 12 & 1548 & 1561\end{array}$

$\begin{array}{lllll}-1 & 2 & 12 & 687 & 707\end{array}$

$\begin{array}{lllll}0 & 2 & 12 & 816 & 828\end{array}$

$\begin{array}{lllll}1 & 2 & 12 & 941 & 955\end{array}$

$\begin{array}{lllll}3 & 2 & 12 & 1890 & 1904\end{array}$

$\begin{array}{lllll}4 & 2 & 12 & 227 & 175\end{array}$

$\begin{array}{lllll}5 & 2 & 12 & 1069 & 1084\end{array}$

$\begin{array}{lllll}6 & 2 & 12 & 312 & 335\end{array}$

$\begin{array}{lllll}7 & 2 & 12 & 430 & 434\end{array}$

$\begin{array}{lllll}8 & 2 & 12 & 928 & 943\end{array}$

$\begin{array}{llllll}9 & 2 & 12 & 416 & 408\end{array}$

$\begin{array}{lllll}10 & 2 & 12 & 443 & 440\end{array}$

$\begin{array}{lllll}-5 & 3 & 12 & 425 & 419\end{array}$

$\begin{array}{lllll}-3 & 3 & 12 & 674 & 670\end{array}$

$\begin{array}{lllll}-2 & 3 & 12 & 640 & 636\end{array}$

$\begin{array}{lllll}-1 & 3 & 12 & 921 & 901\end{array}$

$\begin{array}{lllll}0 & 3 & 12 & 762 & 750\end{array}$

$\begin{array}{lllll}1 & 3 & 12 & 471 & 475\end{array}$

$\begin{array}{lllll}2 & 3 & 12 & 605 & 618\end{array}$

$\begin{array}{lllll}3 & 3 & 12 & 1665 & 1662\end{array}$

$\begin{array}{lllll}4 & 3 & 12 & 721 & 730\end{array}$

$\begin{array}{lllll}5 & 3 & 12 & 1467 & 1508\end{array}$

$\begin{array}{llllll}6 & 3 & 12 & 743 & 758\end{array}$

$\begin{array}{lllll}7 & 3 & 12 & 508 & 492\end{array}$

$\begin{array}{llllll}8 & 3 & 12 & 1150 & 1158\end{array}$

$\begin{array}{lllll}9 & 3 & 12 & 240 & 261\end{array}$ $\mathrm{h} k$ I 10FO 10FC

$\begin{array}{lllll}10 & 3 & 12 & 917 & 944\end{array}$

$\begin{array}{lllll}-8 & 4 & 12 & 518 & 530 \\ -6 & 4 & 12 & 357 & 320\end{array}$

$\begin{array}{lllll}-5 & 4 & 12 & 615 & 650\end{array}$

$\begin{array}{lllll}-4 & 4 & 12 & 866 & 896\end{array}$

$\begin{array}{llllll}-2 & 4 & 12 & 299 & 293\end{array}$

$\begin{array}{lllll}-1 & 4 & 12 & 569 & 547\end{array}$

$\begin{array}{lllll}0 & 4 & 12 & 458 & 477\end{array}$

$\begin{array}{lllll}2 & 4 & 12 & 372 & 354\end{array}$

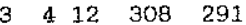

$\begin{array}{lllll}4 & 4 & 12 & 967 & 978\end{array}$

$54121191 \quad 1172$

$\begin{array}{llllll}6 & 4 & 12 & 1056 & 1063\end{array}$

$\begin{array}{lllll}7 & 4 & 12 & 437 & 420\end{array}$

$\begin{array}{llllll}8 & 4 & 12 & 880 & 885\end{array}$

$\begin{array}{lllll}10 & 4 & 12 & 955 & 952\end{array}$

$\begin{array}{lllll}-6 & 5 & 12 & 279 & 271\end{array}$

$\begin{array}{lllll}-5 & 5 & 12 & 667 & 680\end{array}$

$\begin{array}{lllll}-3 & 5 & 12 & 931 & 896\end{array}$

$\begin{array}{lllll}-2 & 5 & 12 & 619 & 617\end{array}$

$\begin{array}{lllll}1 & 5 & 12 & 236 & 228\end{array}$

$\begin{array}{lllll}2 & 5 & 12 & 209 & 169\end{array}$

$\begin{array}{lllll}3 & 5 & 12 & 474 & 492\end{array}$

$\begin{array}{lllll}4 & 5 & 12 & 627 & 648\end{array}$

$\begin{array}{lllll}6 & 5 & 12 & 858 & 881\end{array}$

$\begin{array}{lllll}8 & 5 & 12 & 497 & 549\end{array}$

$\begin{array}{lllll}10 & 5 & 12 & 747 & 757\end{array}$

$\begin{array}{lllll}-6 & 6 & 12 & 352 & 382\end{array}$

$\begin{array}{lllll}-5 & 6 & 12 & 537 & 533\end{array}$

$\begin{array}{lllll}-4 & 6 & 12 & 215 & 225\end{array}$

$\begin{array}{llllll}-3 & 6 & 12 & 1304 & 1346\end{array}$

$\begin{array}{lllll}-1 & 6 & 12 & 1017 & 1026\end{array}$

$\begin{array}{lllll}0 & 6 & 12 & 580 & 587\end{array}$

$\begin{array}{lllll}1 & 6 & 12 & 379 & 392\end{array}$ 


\begin{tabular}{|c|c|c|c|c|c|c|c|c|c|c|c|c|c|c|c|c|c|c|c|c|c|c|}
\hline-6 & 712 & 301 & 295 & 2 & 9 & 12 & 596 & 616 & 312 & 12 & 546 & 596 & 2 & -9 & 13 & 831 & 856 & -3 & -5 & 13 & 467 & 496 \\
\hline-5 & $7 \quad 12$ & 334 & 321 & 3 & 9 & 12 & 653 & 644 & 412 & 12 & 435 & 436 & 3 & -9 & 13 & 458 & 446 & -2 & -5 & 13 & 1373 & 1424 \\
\hline-4 & 712 & 477 & 483 & 4 & 9 & 12 & 1110 & 1118 & -113 & 1.2 & 377 & 367 & -8 & -8 & 13 & 301 & 262 & -1 & -5 & 13 & 574 & 565 \\
\hline-3 & 712 & 907 & 937 & 5 & 9 & 12 & 289 & 263 & 013 & 12 & 421 & 431 & -2 & -8 & 13 & 956 & 991 & 0 & -5 & 13 & 622 & 628 \\
\hline-2 & $7 \quad 12$ & 489 & 506 & 6 & 9 & 12 & 625 & 631 & 113 & 12 & 372 & 401 & -1 & -8 & 13 & 292 & 266 & 1 & -5 & 13 & 871 & 888 \\
\hline-1 & 712 & 1413 & 1472 & 7 & 9 & 12 & 378 & 360 & 313 & 12 & 799 & 829 & 0 & -8 & 13 & 707 & 724 & 2 & -5 & 13 & 222 & 229 \\
\hline 0 & 712 & 646 & 620 & 8 & 9 & 12 & 248 & 278 & 513 & 12 & 719 & 690 & 2 & -8 & 13 & 742 & 768 & 3 & -5 & 13 & 623 & 609 \\
\hline 1 & 712 & 757 & 739 & 9 & 9 & 12 & 1062 & 1032 & 713 & 12 & 318 & 335 & 3 & -8 & 13 & 608 & 610 & 4 & -5 & 13 & 336 & 320 \\
\hline 2 & 712 & 1285 & 1351 & 11 & 9 & 12 & 652 & 645 & 813 & 12 & 278 & 288 & 4 & $-B$ & 13 & 800 & 803 & $-\hat{B}$ & -4 & 13 & 784 & 771 \\
\hline 3 & 712 & 334 & 371 & -4 & 10 & 12 & 420 & 407 & o 14 & 12 & 287 & 327 & 5 & -8 & 13 & 534 & 499 & -6 & -4 & 13 & 897 & 894 \\
\hline 4 & $7 \quad 12$ & 921 & 919 & -3 & 10 & 12 & 343 & 311 & 114 & 12 & 489 & 477 & -6 & -7 & 13 & 225 & 237 & -4 & -4 & 13 & 1188 & 1183 \\
\hline 6 & $\begin{array}{ll}7 & 12\end{array}$ & 346 & 339 & 1 & 10 & 12 & 276 & 303 & 314 & 12 & 670 & 662 & -4 & -7 & 13 & 510 & 542 & -3 & -4 & 13 & 686 & 720 \\
\hline 7 & $\begin{array}{ll}7 & 12\end{array}$ & 640 & 625 & 3 & 10 & 12 & 434 & 476 & 514 & 12 & 677 & 682 & -3 & -7 & 13 & 235 & 259 & -2 & -4 & 13 & 1566 & 1559 \\
\hline 8 & $\begin{array}{ll}712 \\
12\end{array}$ & 668 & 634 & 4 & 10 & 12 & 443 & 439 & 714 & 12 & 415 & 411 & -2 & -7 & 13 & 294 & 278 & -1 & -4 & 13 & 397 & 386 \\
\hline 9 & 712 & 208 & 194 & 5 & 10 & 12 & 856 & 859 & 814 & 12 & 440 & 450 & 0 & -7 & 13 & 562 & 581 & 0 & -4 & 13 & 764 & 763 \\
\hline 10 & 712 & 416 & 351 & 6 & 10 & 12 & 640 & 621 & 315 & 12 & 287 & 330 & 2 & -7 & 13 & 320 & 346 & 1 & -4 & 13 & 1087 & 1103 \\
\hline-4 & $8 \quad 12$ & 354 & 360 & 7 & 10 & 12 & 549 & 550 & 515 & 12 & 497 & 496 & 3 & -7 & 13 & 459 & 459 & 3 & -4 & 13 & 1336 & 1334 \\
\hline-3 & 812 & 374 & 426 & 9 & 10 & 12 & 761 & 745 & $-3-11$ & 13 & 819 & 809 & 4 & -7 & 13 & 561 & 521 & 4 & -4 & 13 & 202 & 204 \\
\hline-2 & 812 & 800 & 804 & 10 & 10 & 12 & 259 & 249 & $-1-11$ & 13 & 340 & 340 & 5 & -7 & 13 & 535 & 492 & 5 & -4 & 13 & 642 & 645 \\
\hline-1 & $8 \quad 12$ & 934 & 958 & 11 & 10 & 12 & 718 & 675 & $0-11$ & 13 & 227 & 313 & 6 & -7 & 13 & 292 & 283 & 8 & -4 & 13 & 302 & 340 \\
\hline 0 & $8 \quad 12$ & 716 & 757 & -2 & 11 & 12 & 494 & 505 & $-5-10$ & 13 & 685 & 673 & -8 & -6 & 13 & 496 & 523 & -9 & -3 & 13 & 304 & 263 \\
\hline 1 & 812 & 542 & 585 & -1 & 11 & 12 & 238 & 277 & $-4-10$ & 13 & 386 & 423 & -7 & -6 & 13 & 453 & 438 & -8 & -3 & 13 & 244 & 271 \\
\hline 2 & 812 & 1083 & 1114 & 1 & 11 & 12 & 356 & 345 & $-3-10$ & 13 & 880 & 890 & -6 & -6 & 13 & 409 & 410 & -6 & -3 & 13 & 515 & 514 \\
\hline 4 & 812 & 1191 & 1208 & 2 & 11 & 12 & 343 & 324 & $-2-10$ & 13 & 364 & 346 & -5 & -6 & 13 & 341 & 362 & -5 & -3 & 13 & 223 & 174 \\
\hline 6 & 812 & 616 & 640 & 4 & 11 & 12 & 273 & 256 & $-1-10$ & 13 & 451 & 433 & -4 & -6 & 13 & 1096 & 1104 & -4 & -3 & 13 & 580 & 582 \\
\hline & & & & 5 & 11 & 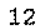 & & & & & & & -2 & & & & & & & & 1039 & 108 \\
\hline
\end{tabular}

Observed and calculated structure factors: [OS(CO) $\left.{ }_{2} \mathrm{PPH}_{3}\right]_{2}$ (MU-I) (MU-PPH $\left.{ }_{2}\right)$

Page 32

h $k I$ IOFO 10Fc

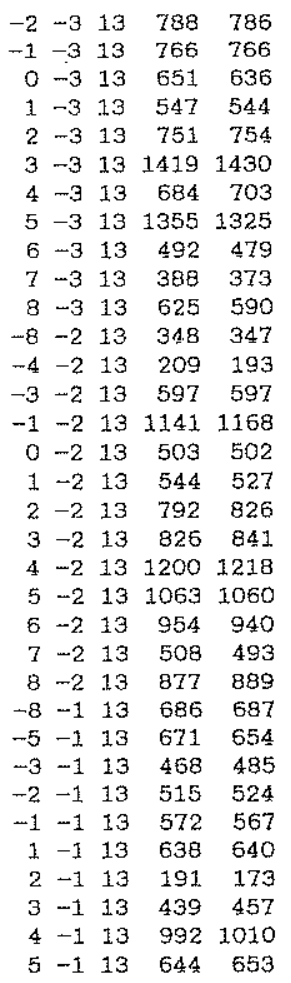

$\mathrm{h} k 1$ 10FO 1OFC

$\begin{array}{lllll}6 & -1 & 13 & 1047 & 1072\end{array}$

$\begin{array}{lllll}7 & -1 & 13 & 225 & 267\end{array}$

$\begin{array}{lllll}8 & -1 & 13 & 683 & 684\end{array}$

$\begin{array}{lllll}-9 & 0 & 13 & 474 & 458\end{array}$

$\begin{array}{lllll}-8 & 0 & 13 & 795 & 778\end{array}$

$\begin{array}{lllll}-7 & 0 & 13 & 481 & 506\end{array}$

$\begin{array}{lllll}-6 & 0 & 13 & 266 & 270\end{array}$

$\begin{array}{lllll}-5 & 0 & 13 & 770 & 802\end{array}$

$\begin{array}{lllll}-4 & 0 & 13 & 530 & 550\end{array}$

$\begin{array}{lllll}-3 & 0 & 13 & 1661 & 1696\end{array}$

$\begin{array}{lllll}-1 & 0 & 13 & 802 & 802\end{array}$

$\begin{array}{lllll}1 & 0 & 13 & 228 & 237\end{array}$

$\begin{array}{lllll}2 & 0 & 13 & 643 & 665\end{array}$

$\begin{array}{llllll}3 & 0 & 13 & 194 & 204\end{array}$

$\begin{array}{llllll}6 & 0 & 13 & 434 & 443\end{array}$

$\begin{array}{lllll}7 & 0 & 13 & 237 & 182\end{array}$

$\begin{array}{lllll}8 & 0 & 13 & 201 & 162\end{array}$

$\begin{array}{lllll}9 & 0 & 13 & 389 & 386\end{array}$

$\begin{array}{lllll}10 & 0 & 13 & 371 & 418\end{array}$

$\begin{array}{lllll}-8 & 1 & 13 & 450 & 423\end{array}$

$\begin{array}{lllll}-7 & 1 & 13 & 860 & 857\end{array}$

$\begin{array}{lllll}-6 & 1 & 13 & 309 & 295\end{array}$

$\begin{array}{lllll}-5 & 1 & 13 & 767 & 755\end{array}$

$\begin{array}{lllll}-4 & 1 & 13 & 842 & 829\end{array}$

$\begin{array}{lllll}-3 & 1 & 13 & 1532 & 1530\end{array}$

$\begin{array}{lllll}-2 & 1 & 13 & 813 & 821\end{array}$

$\begin{array}{lllll}-1 & 1 & 13 & 1543 & 1597\end{array}$

$\begin{array}{llllll}0 & 1 & 13 & 625 & 601\end{array}$

$\begin{array}{lllll}1 & 1 & 13 & 662 & 668\end{array}$

$\begin{array}{lllll}2 & 1 & 13 & 1286 & 1269\end{array}$

$\begin{array}{llllll}3 & 1 & 13 & 480 & 476\end{array}$

$\begin{array}{lllll}4 & 1 & 13 & 710 & 698\end{array}$

$\begin{array}{llllll}5 & 1 & 13 & 244 & 248\end{array}$

$\begin{array}{lllll}8 & 1 & 13 & 325 & 275\end{array}$ $\mathrm{h} k \mathrm{k}$ 10F' 10FC

$\begin{array}{lllll}-7 & 2 & 13 & 904 & 907\end{array}$

$\begin{array}{lllll}-6 & 2 & 13 & 222 & 139\end{array}$

$\begin{array}{lllll}-5 & 2 & 13 & 740 & 740\end{array}$

$\begin{array}{lllll}-4 & 2 & 13 & 395 & 409\end{array}$

$\begin{array}{lllll}-3 & 2 & 13 & 1144 & 1166\end{array}$

$\begin{array}{lllll}-2 & 2 & 13 & 1220 & 1223\end{array}$

$\begin{array}{lllll}-1 & 2 & 13 & 1529 & 1530\end{array}$

$\begin{array}{llllll}0 & 2 & 13 & 1096 & 1074\end{array}$

$\begin{array}{lllll}1 & 2 & 13 & 419 & 408\end{array}$

$\begin{array}{llllll}2 & 2 & 13 & 1323 & 1361\end{array}$

$\begin{array}{llllll}3 & 2 & 13 & 211 & 228\end{array}$

$\begin{array}{lllll}4 & 2 & 13 & 1516 & 1530\end{array}$

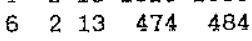

$\begin{array}{lllll}7 & 2 & 13 & 498 & 497\end{array}$

$\begin{array}{lllll}8 & 2 & 13 & 291 & 314\end{array}$

$\begin{array}{lllll}9 & 2 & 13 & 392 & 401\end{array}$

$\begin{array}{lllll}10 & 2 & 13 & 359 & 358\end{array}$

$\begin{array}{lllll}-7 & 3 & 13 & 273 & 271\end{array}$

$\begin{array}{lllll}-5 & 3 & 13 & 414 & 387\end{array}$

$\begin{array}{llllll}-3 & 3 & 13 & 503 & 523\end{array}$

$\begin{array}{lllll}-2 & 3 & 13 & 1109 & 1107\end{array}$

$\begin{array}{lllll}-1 & 3 & 13 & 905 & 895\end{array}$

$\begin{array}{lllll}0 & 3 & 13 & 931 & 943\end{array}$

$\begin{array}{lllll}1 & 3 & 13 & 450 & 430\end{array}$

$\begin{array}{lllll}2 & 3 & 13 & 813 & 836\end{array}$

$\begin{array}{lllll}3 & 3 & 13 & 712 & 706\end{array}$

$\begin{array}{llllll}4 & 3 & 13 & 1526 & 1515\end{array}$

$\begin{array}{llllll}5 & 3 & 13 & 777 & 791\end{array}$

$\begin{array}{lllll}6 & 3 & 13 & 931 & 957\end{array}$

$\begin{array}{lllll}7 & 3 & 13 & 787 & 814\end{array}$

$\begin{array}{lllll}9 & 3 & 13 & 924 & 954\end{array}$

$\begin{array}{lllll}10 & 3 & 13 & 209 & 101\end{array}$

$\begin{array}{lllll}11 & 3 & 13 & 500 & 494\end{array}$

$\begin{array}{lllll}-7 & 4 & 13 & 332 & 315\end{array}$ $\mathrm{h} k \mathrm{k} 1 \mathrm{OFO} 10 \mathrm{Fc}$

$\begin{array}{rllll}-5 & 4 & 231 & 165\end{array}$ $\begin{array}{lllll}-4 & 4 & 13 & 267 & 275\end{array}$

$\begin{array}{lllll}-3 & 4 & 13 & 228 & 256\end{array}$

$\begin{array}{lllll}-2 & 4 & 13 & 595 & 588\end{array}$

$\begin{array}{lllll}-1 & 4 & 13 & 223 & 189\end{array}$

$\begin{array}{lllll}0 & 4 & 13 & 865 & 864\end{array}$

$\begin{array}{lllll}1 & 4 & 13 & 369 & 362\end{array}$

$\begin{array}{lllll}2 & 4 & 13 & 183 & 170\end{array}$

$\begin{array}{lllll}3 & 4 & 13 & 1105 & 1119\end{array}$

$\begin{array}{lllll}4 & 4 & 13 & 882 & 882\end{array}$

$5 \quad 4 \quad 131361 \quad 1387$

$\begin{array}{lllll}6 & 4 & 13 & 906 & 925\end{array}$

$\begin{array}{lllll}7 & 4 & 13 & 880 & 903\end{array}$

$\begin{array}{llllll}8 & 4 & 13 & 230 & 236\end{array}$

$\begin{array}{llllll}9 & 4 & 13 & 1026 & 1050\end{array}$

11. $413 \quad 787 \quad 782$

$\begin{array}{lllll}-7 & 5 & 13 & 540 & 524\end{array}$

$\begin{array}{lllll}-4 & 5 & 13 & 578 & 567\end{array}$

$\begin{array}{lllll}-3 & 5 & 13 & 310 & 311\end{array}$

$\begin{array}{lllll}-2 & 5 & 13 & 285 & 291\end{array}$

$\begin{array}{lllll}-1 & 5 & 13 & 398 & 402\end{array}$

\begin{tabular}{lllll}
\hline & 5 & 13 & 508 & 497
\end{tabular}

$\begin{array}{lllll}1 & 5 & 13 & 324 & 325\end{array}$

$\begin{array}{llllll}3 & 5 & 13 & 458 & 451\end{array}$

$\begin{array}{llllll}5 & 5 & 13 & 1290 & 1275\end{array}$

$\begin{array}{lllll}6 & 5 & 13 & 417 & 408\end{array}$

$\begin{array}{lllll}7 & 5 & 13 & 855 & 867\end{array}$

$\begin{array}{lllll}8 & 5 & 13 & 223 & 270\end{array}$

$\begin{array}{lllll}9 & 5 & 13 & 718 & 705\end{array}$

$\begin{array}{lllll}11 & 5 & 13 & 663 & 644\end{array}$

$\begin{array}{lllll}-7 & 6 & 13 & 549 & 550\end{array}$

$\begin{array}{lllll}-6 & 6 & 13 & 334 & 314\end{array}$

$\begin{array}{lllll}-4 & 6 & 13 & 598 & 588\end{array}$

$\begin{array}{lllll}-3 & 6 & 13 & 410 & 413\end{array}$ h $\mathrm{k} 1$ 10KO 10FC

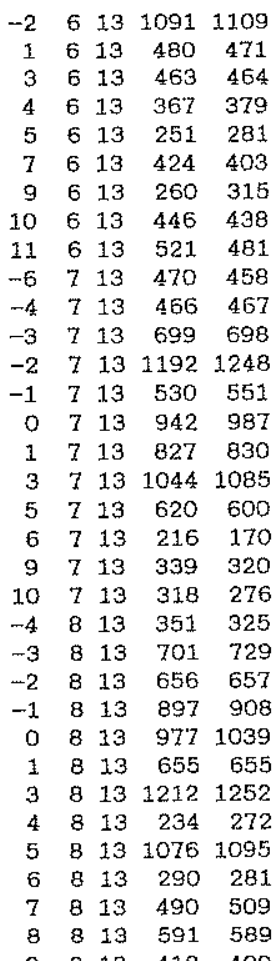

$\begin{array}{llllll}9 & 8 & 13 & 418 & 400\end{array}$ 


\begin{tabular}{|c|c|c|c|c|c|c|c|c|c|c|c|c|c|c|c|c|c|c|c|c|c|c|c|}
\hline 9 & 13 & 656 & 643 & 5 & 14 & 13 & 294 & 215 & -4 & -6 & 14 & 348 & 328 & 0 & -3 & 14 & 852 & 843 & -7 & U & 1.4 & 682 & 82 \\
\hline 9 & 13 & 892 & 893 & 6 & 14 & 13 & 471 & 468 & -3 & -6 & 14 & 658 & 672 & 2 & -3 & 14 & 889 & 894 & -6 & 0 & 14 & 310 & 316 \\
\hline 9 & 13 & 424 & 434 & 7 & 14 & 13 & 327 & 316 & -2 & -6 & 14 & 228 & 218 & 3 & -3 & 14 & 698 & 710 & -4 & 0 & 14 & 695 & 724 \\
\hline 9 & 13 & 504 & 556 & $-4-$ & -10 & 14 & 667 & 663 & 0 & -6 & 14 & 307 & 309 & 4 & -3 & 1.4 & 1305 & 1323 & -2 & 0 & 14 & 766 & 775 \\
\hline 9 & 13 & 821 & 807 & -3 & -10 & 14 & 494 & 469 & 1 & -6 & 14 & 404 & 418 & 5 & -3 & 14 & 380 & 341 & -1 & 0 & 14 & 410 & 421 \\
\hline 10 & 13 & 803 & 775 & $-2-$ & -10 & 14 & 647 & 631 & 4 & -6 & 14 & 444 & 445 & 6 & -3 & 14 & 580 & 575 & 0 & 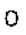 & 14 & 461 & 6.1 \\
\hline $\begin{array}{ll}-1 & 10\end{array}$ & 13 & 359 & 375 & $-1-$ & -10 & 14 & 413 & 413 & 6 & -6 & 14 & 568 & 531 & 7 & -3 & 14 & 228 & 223 & 1 & 0 & 14 & 304 & 102 \\
\hline 110 & 13 & 428 & 424 & & -10 & 14 & 457 & 429 & -8 & -5 & 14 & 364 & 409 & -8 & -2 & 14 & 272 & 221 & 2 & 0 & 14 & 670 & 70 \\
\hline 410 & 13 & 769 & 782 & -6 & -9 & 14 & 731 & 707 & -7 & -5 & 14 & 881. & 861 & -7 & -2 & 14 & 213 & $12 B$ & 3 & 0 & 14 & 222 & 8 \\
\hline 510 & 13 & 659 & 678 & -4 & -9 & 14 & 410 & 403 & -5 & -5 & 14 & 689 & 689 & -6 & -2 & 14 & 206 & 145 & 5 & 0 & 14 & 823 & 351 \\
\hline 610 & 13 & 637 & 624 & -3 & -9 & 14 & 806 & 843 & -4 & -5 & 14 & 532 & 575 & -5 & -2 & 14 & 703 & 682 & 7 & 0 & 14 & 805 & 96 \\
\hline 710 & 13 & 397 & 377 & -2 & -9 & $1 \frac{1}{2}$ & 617 & 579 & -3 & -5 & 14 & 1200 & 1199 & -2 & -2 & 14 & 1000 & 977 & 9 & 0 & 14 & 582 & 54 \\
\hline 810 & 13 & 733 & 742 & -1 & -9 & 14 & 686 & 688 & -2 & -5 & 1.4 & 567 & 531 & 0 & -2 & 14 & 1034 & 1037 & 10 & 0 & 14 & 469 & 57 \\
\hline 1010 & 13 & 816 & 798 & 1 &. .9 & 14 & 594 & 605 & -1 & -5 & 14 & 82.1 & 832 & 2 & -2 & 14 & 646 & 667 & -8 & 1 & 14 & 776 & 93 \\
\hline-311 & 13 & $\triangle 47$ & 446 & 2 & -9 & 14 & 456 & 417 & 0 & -5 & 14 & 218 & 215 & 3 & -2 & 14 & 1269 & 1322 & -7 & 1 & 14 & 355 & 363 \\
\hline 211 & 13 & 231 & 218 & 3 & -9 & 14 & 794 & 810 & 2 & -5 & 14 & 404 & 435 & 4 & -2 & 14 & 872 & 852 & -6 & 1 & 14 & 709 & 717 \\
\hline 311 & 13 & 228 & 222 & -6 & -8 & 14 & 412 & 417 & 6 & -5 & 14 & 306 & 311 & 5 & -2 & 14 & 1222 & 1227 & -4 & 1 & 14 & 686 & 675 \\
\hline 611 & 13 & 617 & 612. & -3 & -8 & 14 & 649 & 662. & -7 & -4 & 14 & 1176 & 1174 & 6 & -2 & 14 & 851 & 858 & -3 & 1 & 14 & 1022 & \\
\hline 811 & 13 & 676 & 652 & -1 & -8 & 14 & 973 & 980 & -5 & -4 & 14 & 970 & 955 & 7 & -2 & 14 & 652 & 689 & -2 & 1 & 14 & 1397 & 1395 \\
\hline 1011 & 13 & 475 & 492 & 1 & -8 & 14 & 770 & 794 & -4 & -4 & 14 & 532 & 527 & 9 & -2 & 14 & 585 & 584 & -1 & 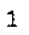 & 14 & 589 & 614 \\
\hline $\begin{array}{ll}-1 & 12\end{array}$ & 13 & 431 & 461 & 2 & -8 & 14 & 451 & 428 & -3 & -4 & 14 & 1069 & 1084 & -7 & -1 & 14 & 558 & 528 & 0 & 1 & 14 & 655 & 622 \\
\hline 212 & 13 & 257 & 283 & 3 & -8 & 14 & 680 & 694 & -2 & -4 & 14 & 975 & 962 & -5 & -1 & 14 & 229 & 255 & 3 & 1 & 14 & 727 & 710 \\
\hline 412 & 13 & 207 & 215 & 4 & -8 & 14 & 689 & 720 & -1 & -4 & 14 & 1270 & 1294 & -4 & -1 & 14 & 368 & 347 & 10 & 1 & 14 & 389 & 395 \\
\hline 512 & 13 & 354 & 340 & -6 & -7 & 14 & 210 & 91 & 0 & -4 & 14 & 972 & 984 & -2 & -1 & 14 & 381 & 365 & -8 & 2 & 14 & 682 & 723 \\
\hline 612 & 13 & 268 & 288 & -2 & -7 & 14 & 346 & 364 & 2 & -4 & 14 & 861 & 874 & -1 & -1 & 14 & 493 & 471 & -6 & 2 & 14 & 953 & 958 \\
\hline 812 & 13 & 275 & 250 & -1 & -7 & 14 & 858 & 878 & 4 & -4 & 14 & 745 & 739 & 0 & -1 & 14 & 1009 & 980 & -4 & 2 & 14 & 660 & 659 \\
\hline
\end{tabular}

h $\mathrm{k} I 10 \mathrm{FO} 10 \mathrm{FC}$

$\begin{array}{lllll}-3 & 2 & 14 & 973 & 973\end{array}$

$\begin{array}{lllll}-2 & 2 & 14 & 1172 & 1184\end{array}$

$\begin{array}{llllll}-1 & 2 & 14 & 1455 & 1467\end{array}$

$\begin{array}{lllll}0 & 2 & 14 & 1108 & 1.100\end{array}$

$\begin{array}{lllll}1 & 2 & 14 & 997 & 986\end{array}$

$\begin{array}{llllll}3 & 2 & 14 & 1089 & 1087\end{array}$

$\begin{array}{lllll}4 & 2 & 14 & 261 & 245\end{array}$

$\begin{array}{lllll}5 & 2 & 14 & 661 & 676\end{array}$

$\begin{array}{lllll}7 & 2 & 14 & 217 & 217\end{array}$

$\begin{array}{lllll}-6 & 3 & 14 & 791 & 798\end{array}$

$\begin{array}{lllll}-5 & 3 & 14 & 213 & 181\end{array}$

$\begin{array}{lllll}-4 & 3 & 14 & 712 & 700\end{array}$

$\begin{array}{lllll}-3 & 3 & 14 & 533 & 523\end{array}$

$\begin{array}{lllll}-2 & 3 & 14 & 540 & 528\end{array}$

$\begin{array}{lllll}-1 & 3 & 14 & 1508 & 1532\end{array}$

$\begin{array}{lllll}0 & 3 & 14 & 918 & 960\end{array}$

$1 \quad 314953 \quad 960$

$\begin{array}{lllll}2 & 3 & 14 & 380 & 409\end{array}$

$\begin{array}{lllll}3 & 3 & 14 & 1072 & 1060\end{array}$

$\begin{array}{lllll}4 & 3 & 14 & 930 & 925\end{array}$

$\begin{array}{lllll}5 & 3 & 14 & 1235 & 1218\end{array}$

$\begin{array}{lllll}6 & 3 & 14 & 501 & 471\end{array}$

$\begin{array}{lllll}7 & 3 & 14 & 401 & 416\end{array}$

$\begin{array}{rrrrr}8 & 3 & 14 & 532 & 545 \\ 10 & 3 & 14 & 486 & 484\end{array}$

$\begin{array}{ccccc}10 & 3 & 14 & 486 & 484 \\ -5 & 4 & 14 & 239 & 195\end{array}$

$\begin{array}{lllll}-4 & 4 & 14 & 314 & 325\end{array}$

$\begin{array}{lllll}-1 & 4 & 14 & 890 & 875\end{array}$

$\begin{array}{lllll}0 & 4 & 14 & 272 & 311\end{array}$

$\begin{array}{lllll}1.4 & 44 & 725 & 717\end{array}$

$\begin{array}{llllll}2 & 4 & 14 & 439 & 426\end{array}$

$\begin{array}{lllll}3 & 4 & 14 & 593 & 616\end{array}$

$4 \quad 41414061419$

$\begin{array}{llllll}5 & 4 & 14 & 858 & 863\end{array}$ h k I 10Fo 10FC

$\begin{array}{lllll}6 & 4 & 14 & 1140 & 1142\end{array}$

$\begin{array}{llllll}7 & 4 & 14 & 597 & 608\end{array}$

$8 \begin{array}{lllll}8 & 4 & 14 & 665 & 653\end{array}$

$\begin{array}{lllll}10 & 4 & 14 & 737 & 764\end{array}$

$\begin{array}{lllll}11 & 4 & 14 & 226 & 202\end{array}$

$\begin{array}{lllll}-6 & 5 & 14 & 260 & 282\end{array}$

$\begin{array}{llllll}-3 & 5 & 14 & 351 & 363\end{array}$

$\begin{array}{llllll}-2 & 5 & 14 & 362 & 368\end{array}$

$\begin{array}{lllll}-1 & 5 & 14 & 259 & 228\end{array}$

$\begin{array}{lllll}0 & 5 & 14 & 310 & 335\end{array}$

$\begin{array}{lllll}1 & 5 & 14 & 505 & 517\end{array}$

$\begin{array}{lllll}2 & 5 & 14 & 256 & 289\end{array}$

$\begin{array}{lllll}4 & 5 & 14 & 1377 & 1414\end{array}$

$\begin{array}{lllll}5 & 5 & 14 & 267 & 269\end{array}$

$\begin{array}{llllll}6 & 5 & 14 & 1388 & 1424\end{array}$

$\begin{array}{lllll}7 & 5 & 14 & 478 & 489\end{array}$

$\begin{array}{lllll}8 & 5 & 14 & 805 & 820\end{array}$

$\begin{array}{lllll}9 & 5 & 14 & 213 & 196\end{array}$

$\begin{array}{lllll}10 & 5 & 14 & 651 & 610\end{array}$

$\begin{array}{lllll}11 & 5 & 14 & 482 & 451\end{array}$

$\begin{array}{lllll}-6 & 6 & 14 & 477 & 453\end{array}$

$\begin{array}{lllll}-5 & 6 & 14 & 294 & 311\end{array}$

$\begin{array}{lllll}-4 & 6 & 14 & 275 & 245\end{array}$

$\begin{array}{lllll}-3 & 6 & 14 & 684 & 674\end{array}$

$\begin{array}{lllll}-1 & 6 & 14 & 458 & 478\end{array}$

$\begin{array}{lllll}0 & 6 & 14 & 438 & 442\end{array}$

$\begin{array}{lllll}1 & 6 & 14 & 259 & 209\end{array}$

$\begin{array}{lllll}3 & 6 & 14 & 238 & 233\end{array}$

$\begin{array}{lllll}4 & 6 & 14 & 295 & 291\end{array}$

$\begin{array}{llllll}5 & 6 & 14 & 239 & 215\end{array}$

$\begin{array}{lllll}6 & 6 & 14 & 955 & 955\end{array}$

$\begin{array}{lllll}8 & 6 & 14 & 849 & 825\end{array}$

$\begin{array}{lllll}10 & 6 & 14 & 363 & 371\end{array}$

$\begin{array}{lllll}11 & 6 & 14 & 509 & 516\end{array}$ h k I 10FO 10FC

$\begin{array}{lllll}-6 & 7 & 14 & 332 & 311\end{array}$

$\begin{array}{llllll}-5 & 7 & 14 & 313 & 353\end{array}$

$\begin{array}{lllll}-4 & 7 & 14 & 236 & 223\end{array}$

$\begin{array}{lllll}-3 & 7 & 14 & 510 & 531\end{array}$

$\begin{array}{rrrrr}-2 & 7 & 14 & 725 & 721\end{array}$

$\begin{array}{lllll}-1 & 7 & 11 & 1038 & 1092\end{array}$

$\begin{array}{lllll}0 & 7 & 14 & 393 & 442\end{array}$

$\begin{array}{lllll}1 & 7 & 14 & 202 & 219\end{array}$

$\begin{array}{lllll}2 & 7 & 1.4 & 455 & 441\end{array}$

$\begin{array}{lllll}3 & 7 & 14 & 395 & 424\end{array}$

$\begin{array}{llllll}4 & 7 & 14 & 761 & 770\end{array}$

$\begin{array}{lllll}8 & 7 & 14 & 344 & 314\end{array}$

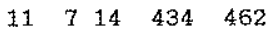

$\begin{array}{lllll}-5 & 8 & 14 & 481 & 473\end{array}$

$\begin{array}{llllll}-4 & 8 & 14 & 296 & 251\end{array}$

$\begin{array}{lllll}-2 & 8 & 14 & 960 & 953\end{array}$

$\begin{array}{lllll}-1 & 8 & 14 & 669 & 683\end{array}$

$\begin{array}{lllll}0 & 8 & 14 & 916 & 930\end{array}$

$\begin{array}{lllll}1 & 8 & 14 & 553 & 597\end{array}$

$\begin{array}{lllll}2 & 8 & 14 & 715 & 747\end{array}$

$\begin{array}{llllll}3 & 8 & 14 & 228 & 229\end{array}$

$\begin{array}{lllll}4 & 8 & 14 & 1012 & 1032\end{array}$

$\begin{array}{llllll}5 & 8 & 14 & 226 & 283\end{array}$

$\begin{array}{lllll}6 & 8 & 14 & 669 & 656\end{array}$

$\begin{array}{llllll}-2 & 9 & 14 & 646 & 654\end{array}$

o $\quad 91411491188$

$\begin{array}{lllll}1 & 9 & 14 & 257 & 274\end{array}$

$\begin{array}{lllll}2 & 9 & 14 & 722 & 763\end{array}$

$\begin{array}{lllll}3 & 9 & 14 & 256 & 257\end{array}$

$\begin{array}{lllll}4 & 9 & 14 & 714 & 729\end{array}$

$\begin{array}{lllll}5 & 9 & 14 & 456 & 465\end{array}$

$\begin{array}{lllll}6 & 9 & 14 & 886 & 889\end{array}$

$\begin{array}{lllll}7 & 9 & 14 & 417 & 463\end{array}$

$\begin{array}{llllll}9 & 9 & 14 & 491 & 494\end{array}$ $\mathrm{h} k \mathrm{k}$ 10Fo $10 \mathrm{FC}$

$\begin{array}{lllll}0 & 10 & 14 & 592 & 617\end{array}$

$\begin{array}{lllll}2 & 10 & 14 & 600 & 600\end{array}$

$\begin{array}{lllll}3 & 10 & 14 & 243 & 296\end{array}$

$\begin{array}{lllll}4 & 10 & 14 & 336 & 336\end{array}$

$\begin{array}{lllll}5 & 10 & 14 & 821 & 822\end{array}$

$\begin{array}{lllll}6 & 10 & 14 & 452 & 465\end{array}$

$\begin{array}{lllll}7 & 10 & 14 & 762 & 758\end{array}$

$\begin{array}{lllll}9 & 10 & 14 & 625 & 612\end{array}$

$\begin{array}{lllll}-2 & 11 & 14 & 281 & 256\end{array}$

$\begin{array}{lllll}5 & 11 & 14 & 677 & 680\end{array}$

$\begin{array}{lllll}7 & 11 & 14 & 867 & 830\end{array}$

$\begin{array}{llllll}9 & 11 & 14 & 577 & 575\end{array}$

$\begin{array}{lllll}10 & 11 & 14 & 425 & 393\end{array}$

$\begin{array}{lllll}\circ & 12 & 14 & 271 & 235\end{array}$

$\begin{array}{lllll}6 & 12 & 14 & 272 & 281\end{array}$

$\begin{array}{lllll}7 & 12 & 14 & 521 & 512\end{array}$

$\begin{array}{lllll}9 & 12 & 14 & 336 & 315\end{array}$

$\begin{array}{lllll}0 & 13 & 14 & 296 & 357\end{array}$

$\begin{array}{llllll}3 & 13 & 14 & 277 & 304\end{array}$

$\begin{array}{lllll}5 & 13 & 14 & 278 & 282\end{array}$

$\begin{array}{lllll}7 & 13 & 14 & 244 & 202\end{array}$

$\begin{array}{lllll}3 & 14 & 14 & 345 & 373\end{array}$

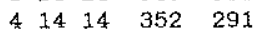

$\begin{array}{lllll}5 & 14 & 14 & 483 & 512\end{array}$

$\begin{array}{lllll}6 & 14 & 14 & 227 & 240\end{array}$

$\begin{array}{llll}-2-10 & 15 & 490 & 503\end{array}$

$\begin{array}{llll}-1-10 & 15 & 351 & 348\end{array}$

$\begin{array}{lllll}-5 & -9 & 15 & 708 & 718\end{array}$

$\begin{array}{lllll}-4 & -9 & 15 & 382 & 385\end{array}$

$\begin{array}{lllll}-3 & -9 & 15 & 431 & 445\end{array}$

$\begin{array}{lllll}-2 & -9 & 15 & 817 & 815\end{array}$

$\begin{array}{lllll}-1 & -9 & 15 & 353 & 335\end{array}$

$\begin{array}{lllll}0 & -9 & 15 & 632 & 610\end{array}$ $\mathrm{h} k \mathrm{k}$ 10FO 10FC

$\begin{array}{lllll}-6 & -8 & 15 & 255 & 249\end{array}$

$\begin{array}{lllll}-5 & -8 & 15 & 559 & 563 \\ -4 & -8 & 15 & 403 & 385\end{array}$

$\begin{array}{lllll}-4 & -8 & 15 & 403 & 385\end{array}$

$\begin{array}{lllll}-2 & -8 & 15 & 1025 & 999\end{array}$

$\begin{array}{lllll}0 & -8 & 15 & 997 & 1040\end{array}$

$\begin{array}{lllll}2 & -8 & 15 & 597 & 584\end{array}$

$\begin{array}{lllll}3 & -8 & 15 & 629 & 626\end{array}$

$\begin{array}{lllll}-5 & -7 & 15 & 243 & 279\end{array}$

$\begin{array}{lllll}-3 & -7 & 15 & 224 & 153\end{array}$

$\begin{array}{lllll}-2 & -7 & 15 & 542 & 530\end{array}$

$\begin{array}{lllll}-1 & -7 & 15 & 323 & 325\end{array}$

$\begin{array}{lllll}0 & -7 & 15 & 1024 & 1006\end{array}$

$\begin{array}{lllll}1 & -7 & 15 & 243 & 208\end{array}$

$\begin{array}{lllll}2 & -7 & 15 & 631 & 637\end{array}$

$\begin{array}{lllll}3 & -7 & 15 & 594 & 572\end{array}$

$\begin{array}{llllll}4 & -7 & 15 & 379 & 367\end{array}$

$\begin{array}{lllll}-7 & -6 & 15 & 257 & 217\end{array}$

$\begin{array}{lllll}-5 & -6 & 15 & 302 & 283\end{array}$

$\begin{array}{lllll}-2 & -6 & 15 & 244 & 235\end{array}$

$\begin{array}{lllll}-1 & -6 & 15 & 704 & 726\end{array}$

$\begin{array}{lllll}0 & -6 & 15 & 569 & 572\end{array}$

$\begin{array}{lllll}1 & -6 & 15 & 365 & 325\end{array}$

$\begin{array}{lllll}2 & -6 & 15 & 518 & 519\end{array}$

$\begin{array}{lllll}3 & -6 & 15 & 378 & 392\end{array}$

$\begin{array}{lllll}5 & -6 & 15 & 752 & 755\end{array}$

$\begin{array}{lllll}-7 & -5 & 15 & 275 & 305\end{array}$

$\begin{array}{lllll}-6 & -5 & 15 & 581 & 586\end{array}$

$\begin{array}{lllll}-4 & -5 & 15 & 426 & 443\end{array}$

$\begin{array}{lllll}-3 & -5 & 15 & 595 & 600\end{array}$

$\begin{array}{lllll}-2 & -5 & 15 & 709 & 671\end{array}$

$\begin{array}{lllll}0 & -5 & 15 & 238 & 183\end{array}$

$\begin{array}{lllll}1 & -5 & 15 & 235 & 182\end{array}$

$\begin{array}{lllll}2 & -5 & 15 & 313 & 311\end{array}$

$\begin{array}{lllll}5 & -5 & 15 & 339 & 341\end{array}$ 


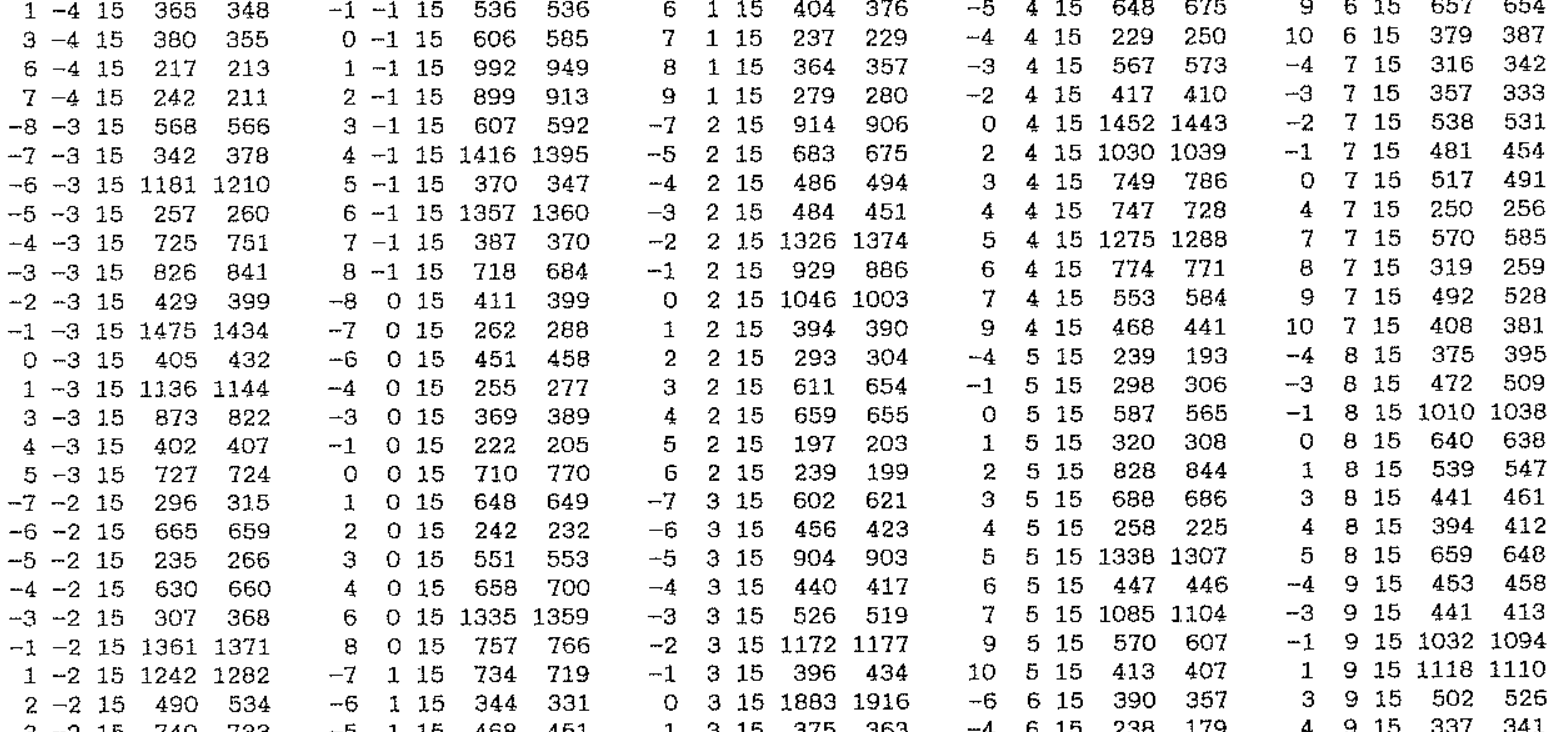

$\begin{array}{lllll}-5 & 1 & 15 & 468 & 451\end{array}$

Observed and calculated structure factors: $\left.\quad \operatorname{lOS}(\mathrm{CO})_{2} \mathrm{PPH}_{3}\right]_{2}(\mathrm{MU}-\mathrm{I})\left(\mathrm{MU}-\mathrm{PPH}_{2}\right)$

Page 36

$\begin{array}{rrrrrrrrrr}\mathrm{h} & \mathrm{k} & 1 & 1 \text { OFO } & 10 \mathrm{Fc} & \mathrm{h} & \mathrm{k} & \mathrm{l} & 1 \text { OFO } & 1 \text { OFc } \\ 5 & 9 & 15 & 595 & 592 & -2 & -7 & 16 & 359 & 333 \\ 6 & 9 & 15 & 483 & 507 & -1 & -7 & 16 & 962 & 971 \\ 7 & 9 & 15 & 570 & 559 & 0 & -7 & 16 & 575 & 554 \\ 8 & 9 & 15 & 297 & 253 & 1 & -7 & 16 & 1030 & 1028 \\ -3 & 10 & 15 & 230 & 231 & 2 & -7 & 16 & 377 & 352 \\ -1 & 10 & 15 & 465 & 471 & 3 & -7 & 16 & 335 & 323 \\ 1 & 10 & 15 & 987 & 1025 & 4 & -7 & 16 & 731 & 717 \\ 3 & 10 & 15 & 592 & 591 & -3 & -6 & 16 & 225 & 201 \\ 4 & 10 & 15 & 573 & 620 & -2 & -6 & 16 & 348 & 352 \\ 5 & 10 & 15 & 440 & 438 & -1 & -6 & 16 & 326 & 335 \\ 6 & 10 & 15 & 905 & 866 & 0 & -6 & 16 & 742 & 740 \\ 7 & 10 & 15 & 416 & 411 & 1 & -6 & 16 & 941 & 926 \\ 8 & 10 & 15 & 502 & 498 & 2 & -6 & 16 & 505 & 511 \\ 10 & 10 & 15 & 371 & 380 & 3 & -6 & 16 & 387 & 407 \\ 1 & 11 & 15 & 316 & 324 & 4 & -6 & 16 & 595 & 601 \\ 4 & 11 & 15 & 575 & 563 & -7 & -5 & 16 & 666 & 641 \\ 6 & 11 & 15 & 913 & 911 & -4 & -5 & 16 & 258 & 229 \\ 8 & 11 & 15 & 736 & 710 & -1 & -5 & 16 & 432 & 447 \\ -1 & 12 & 15 & 297 & 303 & 0 & -5 & 16 & 555 & 547 \\ 6 & 12 & 15 & 630 & 595 & 1 & -5 & 16 & 416 & 406 \\ 7 & 12 & 15 & 277 & 172 & 2 & -5 & 16 & 399 & 402 \\ 8 & 12 & 15 & 660 & 658 & 3 & -5 & 16 & 386 & 363 \\ 1 & 13 & 15 & 268 & 261 & 4 & -5 & 16 & 289 & 295 \\ 4 & 14 & 15 & 233 & 234 & 5 & -5 & 16 & 317 & 288 \\ 5 & 14 & 15 & 237 & 261 & 6 & -5 & 16 & 570 & 558 \\ -3 & -9 & 16 & 457 & 461 & -7 & -4 & 16 & 952 & 972 \\ -1 & -9 & 16 & 693 & 704 & -5 & -4 & 16 & 804 & 842 \\ -5 & -8 & 16 & 399 & 405 & -3 & -4 & 16 & 350 & 330 \\ -4 & -8 & 16 & 356 & 409 & -2 & -4 & 16 & 809 & 818 \\ -3 & -8 & 16 & 586 & 592 & -1 & -4 & 16 & 444 & 427 \\ -1 & -8 & 16 & 1005 & 962 & 0 & -4 & 16 & 384 & 352 \\ 1 & -8 & 16 & 701 & 686 & 3 & -4 & 16 & 327 & 335 \\ -5 & -7 & 16 & 443 & 446 & -7 & -3 & 16 & 862 & 839 \\ -3 & -7 & 16 & 408 & 434 & -6 & -3 & 16 & 574 & 582\end{array}$

h $k 110 \mathrm{FO} 10 \mathrm{FC}$

$\begin{array}{lllll}-5 & -3 & 16 & 1150 & 1144\end{array}$

$\begin{array}{lllll}-4 & -3 & 16 & 343 & 307\end{array}$

$\begin{array}{lllll}-3 & -3 & 16 & 515 & 494\end{array}$

$\begin{array}{lllll}-2 & -3 & 16 & 1044 & 1035\end{array}$

$\begin{array}{lllll}-1 & -3 & 16 & 320 & 264\end{array}$

$\begin{array}{lllll}0 & -3 & 16 & 1094 & 1070\end{array}$

$\begin{array}{lllll}1 & -3 & 16 & 409 & 393\end{array}$

$\begin{array}{lllll}2 & -3 & 16 & 472 & 445\end{array}$

$\begin{array}{lllll}3 & -3 & 16 & 279 & 295\end{array}$

$\begin{array}{lllll}4 & -3 & 16 & 339 & 352\end{array}$

$\begin{array}{lllll}5 & -3 & 16 & 246 & 250\end{array}$

$\begin{array}{lllll}-7 & -2 & 16 & 348 & 294\end{array}$

$\begin{array}{llllll}-6 & -2 & 16 & 810 & 776\end{array}$

$\begin{array}{lllll}-5 & -2 & 16 & 782 & 779\end{array}$

$\begin{array}{lllll}-4 & -2 & 16 & 580 & 603\end{array}$

$\begin{array}{lllll}-3 & -2 & 16 & 442 & 441\end{array}$

$\begin{array}{lllll}-2 & -2 & 16 & 1033 & 1008\end{array}$

$\begin{array}{lllll}-1 & -2 & 16 & 349 & 326\end{array}$

$\begin{array}{lllll}0 & -2 & 16 & 1360 & 1365\end{array}$

$\begin{array}{lllll}1 & -2 & 16 & 302 & 249\end{array}$

$\begin{array}{lllll}2 & -2 & 16 & 815 & 826\end{array}$

$\begin{array}{lllll}3 & -2 & 16 & 483 & 489\end{array}$

$\begin{array}{lllll}4 & -2 & 16 & 465 & 465\end{array}$

$\begin{array}{lllll}5 & -2 & 16 & 693 & 714\end{array}$

$\begin{array}{lllll}6 & -2 & 16 & 404 & 406\end{array}$

$\begin{array}{lllll}7 & -2 & 16 & 275 & 248\end{array}$

$\begin{array}{lllll}-6 & -1 & 16 & 416 & 419\end{array}$

$\begin{array}{lllll}-5 & -1 & 16 & 264 & 273\end{array}$

$\begin{array}{lllll}-4 & -1 & 16 & 463 & 491\end{array}$

$\begin{array}{lllll}-3 & -1 & 16 & 343 & 315\end{array}$

$\begin{array}{lllll}-2 & -1 & 16 & 450 & 462\end{array}$

$\begin{array}{lllll}-1 & -1 & 16 & 562 & 568\end{array}$

$\begin{array}{lllll}0 & -1 & 16 & 1415 & 1411\end{array}$

$\begin{array}{lllll}1 & -1 & 16 & 747 & 745\end{array}$ h k 3 10FO 1OFC

$\begin{array}{lllll}2 & -1 & 16 & 1095 & 1092\end{array}$

$\begin{array}{lllll}3 & -1 & 16 & 1017 & 1043\end{array}$

$\begin{array}{lllll}4 & -1 & 16 & 303 & 310\end{array}$

$\begin{array}{lllll}5 & -1 & 16 & 1156 & 1176\end{array}$

$\begin{array}{llllll}6 & -1 & 16 & 345 & 371\end{array}$

$\begin{array}{lllll}7 & -1 & 16 & 752 & 755\end{array}$

$\begin{array}{lllll}-1 & 0 & 16 & 441 & 446\end{array}$

o $016 \quad 377 \quad 361$

$\begin{array}{lllll}1 & 0 & 16 & 848 & 843\end{array}$

$\begin{array}{lllll}2 & 0 & 16 & 729 & 727\end{array}$

$\begin{array}{lllll}3 & 0 & 16 & 798 & 826\end{array}$

$\begin{array}{lllll}5 & 0 & 16 & 109 \pm & 1.11\end{array}$

$\begin{array}{lllll}6 & 0 & 16 & 206 & 164\end{array}$

$7 \quad 0 \quad 16 \quad 1180 \quad 1166$

$\begin{array}{llllr}9 & 0 & 16 & 578 & 567\end{array}$

$\begin{array}{lllll}-6 & 1 & 16 & 599 & 608\end{array}$

$\begin{array}{lllll}-4 & 1 & 16 & 236 & 212\end{array}$

$\begin{array}{lllll}-3 & 1 & 16 & 308 & 340\end{array}$

$\begin{array}{lllll}-2 & 1 & 16 & 310 & 304\end{array}$

o $1116 \quad 444 \quad 406$

$\begin{array}{lllll}1 & 1 & 16 & 828 & 835\end{array}$

$\begin{array}{lllll}2 & 1 & 16 & 779 & 771\end{array}$

$\begin{array}{lllll}3 & 1 & 16 & 529 & 534\end{array}$

$4 \quad 116 \quad 265 \quad 245$

$\begin{array}{lllll}5 & 1 & 16 & 441 & 456\end{array}$

$\begin{array}{lllll}6 & 1 & 16 & 526 & 511\end{array}$

$\begin{array}{lllll}7 & 1 & 16 & 700 & 697\end{array}$

$\begin{array}{lllll}8 & 1 & 16 & 372 & 381\end{array}$

$\begin{array}{lllll}9 & 1 & 16 & 475 & 525\end{array}$

$\begin{array}{lllll}-6 & 2 & 16 & 878 & 893\end{array}$

$\begin{array}{lllll}-4 & 2 & 16 & 524 & 536\end{array}$

$\begin{array}{lllll}-3 & 2 & 16 & 593 & 607\end{array}$

$\begin{array}{lllll}-1 & 2 & 16 & 807 & 812\end{array}$

$\begin{array}{lllll}0 & 2 & 16 & 571 & 586\end{array}$ h $k$ l 10Fo 10Fc

$\begin{array}{lllll}1 & 2 & 16 & 273 & 242\end{array}$

$\begin{array}{lllll}4 & 2 & 16 & 433 & 451\end{array}$

$\begin{array}{lllll}6 & 2 & 16 & 281 & 237\end{array}$

$8 \quad 2 \quad 16 \quad 534 \quad 526$

$\begin{array}{lllll}9 & 2 & 1.6 & 235 & 237\end{array}$

$\begin{array}{lllll}-6 & 3 & 16 & 850 & 829\end{array}$

$\begin{array}{lllll}-5 & 3 & 16 & 527 & 511\end{array}$

$\begin{array}{lllll}-4 & 3 & 16 & 755 & 780\end{array}$

$\begin{array}{lllll}-3 & 3 & 16 & 529 & 537\end{array}$

$\begin{array}{lllll}-1 & 3 & 16 & 1354 & 1355\end{array}$

131611171140

$\begin{array}{lllll}3 & 3 & 16 & 443 & 432\end{array}$

$4316 \quad 713 \quad 713$

$\begin{array}{lllll}5 & 3 & 16 & 478 & 495\end{array}$

$\begin{array}{lllll}6 & 3 & 1.6 & 343 & 350\end{array}$

$\begin{array}{lllll}7 & 3 & 16 & 387 & 369\end{array}$

$\begin{array}{lllll}8 & 3 & 16 & 223 & 221\end{array}$

$\begin{array}{lllll}10 & 3 & 16 & 234 & 234\end{array}$

$\begin{array}{lllll}-6 & 4 & 16 & 347 & 341\end{array}$

$-5 \quad 16 \quad 700 \quad 717$

$\begin{array}{lllll}-4 & 4 & 16 & 782 & 792\end{array}$

$\begin{array}{lllll}-3 & 4 & 16 & 374 & 407\end{array}$

$-2 \quad 4 \quad 16 \quad 196 \quad 145$

$-141611021090$

$04 \quad 16 \quad 500 \quad 464$

$14161507 \quad 1533$

$\begin{array}{lllll}2 & 4 & 16 & 368 & 362\end{array}$

341610341053

$4 \quad 416 \quad 677 \quad 628$

$\begin{array}{lllll}5 & 4 & 16 & 539 & 525\end{array}$

$64416 \quad 910 \quad 944$

$\begin{array}{lllll}7 & 4 & 16 & 349 & 354\end{array}$

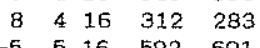




\begin{tabular}{|c|c|c|c|c|c|c|c|c|c|c|c|c|c|c|c|c|c|c|c|c|c|c|c|}
\hline 4 & 516 & 602 & 591 & 5 & 8 & 16 & 280 & 300 & -2 & -8 & 17 & 603 & 568 & -3 & -3 & 17 & 354 & 400 & 7 & 0 & 17 & 332 & 284 \\
\hline 6 & $\begin{array}{ll}5 & 16\end{array}$ & 1109 & 1103 & 9 & 8 & 16 & 342 & 315 & -1 & -8 & 17 & 246 & $24 \mathrm{~B}$ & -1 & -3 & 17 & 780 & 764 & 8 & 0 & 17 & 765 & 771 \\
\hline 8 & 516 & 602 & 606 & -3 & 9 & 16 & 337 & 307 & 0 & -8 & 17 & 776 & 740 & 1 & -3 & 17 & 720 & 698 & -5 & 1 & 17 & 472 & 470 \\
\hline 9 & 516 & 293 & 281 & -2 & 9 & 16 & 591 & 590 & -4 & -7 & 17 & 620 & 643 & 4 & -3 & 17 & 214 & 185 & 0 & 1 & 17 & 458 & 449 \\
\hline 10 & 516 & 386 & 386 & 0 & 9 & 16 & 990 & 1020 & -2 & -7 & 17 & 725 & 710 & -6 & -2 & 17 & 666 & 656 & 1 & 1 & 17 & 300 & 284 \\
\hline-2 & 616 & 246 & 191 & 2 & 9 & 16 & 488 & 501 & -1 & -7 & 17 & 370 & 372 & -5 & -2 & 17 & 903 & 911 & 2 & 1 & 17 & 962 & 957 \\
\hline 0 & 616 & 423 & 408 & 5 & 9 & 16 & 649 & 644 & 0 & -7 & 17 & 796 & 810 & -4 & -2 & 17 & 822 & 830 & 3 & 1 & 17 & 627 & 673 \\
\hline 2 & 616 & 906 & 930 & 6 & 9 & 16 & 350 & 360 & 1. & -7 & 17 & 474 & 502 & -3 & -2 & 17 & 622 & 622 & 4 & 1 & 17 & 567 & 588 \\
\hline 3 & 616 & 287 & 284 & 7 & 9 & 16 & 354 & 344 & 2 & -7 & 17 & 500 & 529 & -1 & -2 & 17 & 897 & 906 & 6 & 1 & 17 & 648 & 521 \\
\hline 4 & 616 & 705 & 730 & 8 & 9 & 16 & 245 & 262 & -4 & -6 & 17 & 550 & 573 & 0 & -2 & 17 & 523 & 457 & 7 & 1 & 17 & 581 & 573 \\
\hline 6 & 616 & 1033 & 1058 & -2 & 10 & 16 & 386 & 399 & -2 & -6 & 17 & 458 & 4.70 & 1 & -2 & 17 & 1134 & 1170 & 8 & 1 & 17 & 769 & 757 \\
\hline 7 & 616 & 231 & 192 & 0 & 10 & 16 & 777 & 813 & -1 & -6 & 17 & 541 & 568 & 3 & -2 & 17 & 382 & 358 & 9 & 1 & 17 & 467 & 465 \\
\hline 8 & 616 & 971 & 953 & 1 & 10 & 16 & 318 & 268 & 0 & -6 & 17 & 760 & 746 & 4 & -2 & 17 & 470 & 467 & -6 & 2 & 17 & 231 & 294 \\
\hline 9 & 616 & 360 & 334 & 2 & 10 & 16 & 912 & 937 & 1 & -6 & 17 & 871 & 867 & 5 & -2 & 17 & 216 & 217 & -5 & 2 & 17 & 774 & 808 \\
\hline 10 & 616 & 379 & 371 & 4 & 10 & 16 & 455 & 411 & 2 & -6 & 17 & 660 & 642 & 6 & -2 & 17 & 235 & 263 & -2 & 2 & 17 & 325 & 332 \\
\hline-5 & 716 & 438 & 442 & 5 & 10 & 16 & 612 & 586 & 3 & -6 & 17 & 538 & 533 & 7 & -2 & 17 & 230 & 226 & -1 & 2 & 17 & 225 & 139 \\
\hline-3 & 716 & 215 & 205 & 6 & 10 & 16 & 221 & 215 & -6 & -5 & 17 & 357 & 316 & -5 & -1 & 17 & 823 & 794 & 0 & 2 & 17 & 312 & 279 \\
\hline-2 & 716 & 340 & 354 & 7 & 10 & 16 & 684 & 663 & -4 & -5 & 17 & 239 & 238 & -4 & -1 & 17 & 384 & 397 & 1 & 2 & 17 & 367 & 346 \\
\hline-1 & 716 & 349 & 344 & 8 & 10 & 16 & 239 & 247 & -1 & -5 & 17 & 296 & 283 & -3 & -1 & 17 & 658 & 676 & 2 & 2 & 17 & 541 & 557 \\
\hline 1 & 716 & 268 & 297 & 9 & 10 & 16 & 254 & 241 & 0 & -5 & 17 & 267 & 233 & -1 & -1 & 17 & 742 & 754 & 3 & 2 & 17 & 313 & 31.4 \\
\hline 2 & 716 & 573 & 566 & 1 & 11 & 16 & 432 & 421 & 1 & -5 & 17 & 755 & 791 & 0 & -1 & 17 & 931 & 878 & 4 & 2 & 17 & 283 & 268 \\
\hline 3 & 716 & 332 & 298 & 2 & 11 & 16 & 648 & 648 & 2 & -5 & 17 & 413 & 453 & 1 & -1 & 17 & 1179 & 1174 & 7 & 2 & 17 & 524 & 527 \\
\hline 5 & 716 & 226 & 208 & 3 & 11 & 16 & 309 & 329 & 3 & -5 & 17 & 602 & 624 & 2 & -1 & 17 & 757 & 780 & 8 & 2 & 17 & 314 & 334 \\
\hline 6 & 716 & 396 & 435 & 4 & 11 & 16 & 270 & 301 & 5 & -5 & 17 & 466 & 479 & 3 & -1 & 17 & 612 & 631 & 9 & 2 & 17 & 538 & 546 \\
\hline 7 & 716 & 455 & 451 & 5 & 11 & 16 & 725 & 722 & -6 & -4 & 17 & 735 & 746 & 6 & -1 & 17 & 707 & 703 & -6 & 3 & 17 & 591 & 576 \\
\hline 8 & 716 & 804 & 819 & 7 & 11 & 16 & 764 & 768 & -4 & -4 & 17 & 282 & 264 & 8 & -1 & 17 & 399 & 375 & -5 & 3 & 17 & 728 & 740 \\
\hline
\end{tabular}

Observed and calculated structure factors: $\quad\left[\mathrm{OS}(\mathrm{CO}){ }_{2} \mathrm{PPH}_{3}\right]_{2}(\mathrm{MU}-\mathrm{I})\left(\mathrm{MJ}-\mathrm{PPH}_{2}\right)$

Page 38

h $k$ l 10FO 10FC

$\begin{array}{rrrrr}-4 & 3 & 17 & 543 & 549 \\ -3 & 3 & 17 & 395 & 444 \\ -2 & 3 & 17 & 550 & 659 \\ 0 & 3 & 17 & 1018 & 1002 \\ 2 & 3 & 17 & 586 & 542 \\ 5 & 3 & 17 & 390 & 390 \\ 9 & 3 & 17 & 506 & 466 \\ -5 & 4 & 17 & 483 & 521 \\ -4 & 4 & 17 & 790 & 809 \\ -3 & 4 & 17 & 600 & 639 \\ -2 & 4 & 17 & 580 & 610 \\ -1 & 4 & 17 & 368 & 353 \\ 0 & 4 & 17 & 1157 & 1170 \\ 1 & 4 & 17 & 370 & 353 \\ 2 & 4 & 17 & 1159 & 1155 \\ 4 & 4 & 17 & 334 & 354 \\ 5 & 4 & 17 & 628 & 639 \\ 7 & 4 & 17 & 445 & 442 \\ -4 & 5 & 17 & 881 & 878 \\ -3 & 5 & 17 & 384 & 415 \\ -2 & 5 & 17 & 554 & 547 \\ -1 & 5 & 17 & 261 & 246 \\ 0 & 5 & 17 & 765 & 803 \\ 1 & 5 & 17 & 882 & 897 \\ 2 & 5 & 17 & 933 & 906 \\ 3 & 5 & 17 & 651 & 647 \\ 4 & 5 & 17 & 438 & 462 \\ 5 & 5 & 17 & 698 & 685 \\ 7 & 5 & 17 & 724 & 744 \\ 9 & 5 & 17 & 305 & 304 \\ -4 & 6 & 17 & 383 & 376 \\ -2 & 6 & 17 & 312 & 332 \\ 0 & 6 & 17 & 248 & 263 \\ 1 & 6 & 17 & 890 & 905\end{array}$

$\mathrm{h} k \mathrm{k} 1$ 10FO 10FC

$2 \quad 6 \quad 17 \quad 504 \quad 515$

$\begin{array}{lllll}3 & 6 & 17 & 855 & 893\end{array}$

$\begin{array}{lllll}5 & 6 & 17 & 727 & 739\end{array}$

$\begin{array}{lllll}7 & 6 & 17 & 892 & 906\end{array}$

$\begin{array}{lllll}8 & 6 & 17 & 240 & 279\end{array}$

$\begin{array}{lllll}9 & 6 & 17 & 463 & 464\end{array}$

$\begin{array}{llllll}-4 & 7 & 17 & 253 & 276\end{array}$

$\begin{array}{lllll}1 & 7 & 17 & 417 & 364\end{array}$

$\begin{array}{lllll}3 & 7 & 17 & 750 & 778\end{array}$

$\begin{array}{lllll}5 & 7 & 17 & 500 & 616\end{array}$

$\begin{array}{lllll}6 & 7 & 17 & 313 & 307\end{array}$

$\begin{array}{lllll}7 & 7 & 17 & 688 & 684\end{array}$

$\begin{array}{lllll}8 & 7 & 17 & 623 & 644\end{array}$

$\begin{array}{lllll}9 & 7 & 17 & 600 & 590\end{array}$

$\begin{array}{lllll}-1 & 8 & 17 & 331 & 343\end{array}$

$\begin{array}{lllll}0 & 8 & 17 & 246 & 243\end{array}$

$\begin{array}{lllll}1 & 8 & 17 & 283 & 269\end{array}$

$\begin{array}{lllll}2 & 8 & 17 & 218 & 198\end{array}$

$\begin{array}{lllll}3 & 8 & 17 & 378 & 380\end{array}$

$\begin{array}{lllll}4 & 8 & 17 & 266 & 290\end{array}$

$\begin{array}{llllll}8 & 8 & 17 & 586 & 558\end{array}$

$\begin{array}{lllll}9 & 8 & 17 & 346 & 305\end{array}$

$\begin{array}{lllll}-3 & 9 & 17 & 360 & 328\end{array}$

$\begin{array}{lllll}-1 & 9 & 17 & 472 & 479\end{array}$

$\begin{array}{lllll}1 & 9 & 17 & 531 & 561\end{array}$

$\begin{array}{lllll}4 & 9 & 17 & 301 & 308\end{array}$

$\begin{array}{lllll}6 & 9 & 17 & 323 & 343\end{array}$

$\begin{array}{lllll}7 & 9 & 17 & 273 & 286\end{array}$

$\begin{array}{lllll}-1 & 10 & 17 & 462 & 451\end{array}$

$\begin{array}{lllll}0 & 10 & 17 & 362 & 367\end{array}$

$\begin{array}{lllll}1 & 10 & 17 & 700 & 706\end{array}$

$\begin{array}{lllll}3 & 10 & 17 & 559 & 575\end{array}$

$\begin{array}{lllll}4 & 10 & 17 & 209 & 267\end{array}$

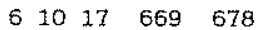

$\mathrm{h}$ k 1 10Fo $10 \mathrm{Fc}$

$\begin{array}{lllll}8 & 10 & 17 & 375 & 369\end{array}$

$\begin{array}{lllll}0 & 11 & 17 & 363 & 360\end{array}$

$\begin{array}{lllll}1 & 11 & 17 & 392 & 411\end{array}$

$\begin{array}{lllll}2 & 11 & 17 & 601 & 609\end{array}$

$\begin{array}{lllll}3 & 11 & 17 & 655 & 675\end{array}$

$\begin{array}{lllll}4 & 11 & 17 & 522 & 462\end{array}$

$\begin{array}{lllll}5 & 11 & 17 & 217 & 229\end{array}$

$\begin{array}{lllll}6 & 111 & 17 & 594 & 610\end{array}$

$\begin{array}{lllll}8 & 11 & 17 & 481 & 491\end{array}$

$\begin{array}{lllll}2 & 12 & 17 & 508 & 509\end{array}$

$\begin{array}{lllll}4 & 12 & 17 & 506 & 523\end{array}$

$\begin{array}{lllll}6 & 12 & 17 & 469 & 473\end{array}$

$\begin{array}{lllll}7 & 12 & 17 & 333 & 259\end{array}$

$\begin{array}{lllll}-3 & -7 & 18 & 613 & 641\end{array}$

$\begin{array}{lllll}-1 & -7 & 18 & 474 & 517\end{array}$

$\begin{array}{lllll}0 & -7 & 18 & 625 & 597\end{array}$

$\begin{array}{lllll}1 & -7 & 18 & 629 & 634\end{array}$

$\begin{array}{lllll}-3 & -6 & 18 & 768 & 753\end{array}$

$\begin{array}{lllll}-2 & -6 & 18 & 245 & 212\end{array}$

$\begin{array}{lllll}-1 & -6 & 18 & 602 & 582\end{array}$

$\begin{array}{llll}-5 & 18 & 781 & 760\end{array}$

$\begin{array}{lllll}1 & -6 & 18 & 548 & 529\end{array}$

$\begin{array}{lllll}2 & -6 & 18 & 713 & 726\end{array}$

$\begin{array}{lllll}3 & -6 & 18 & 315 & 315\end{array}$

$\begin{array}{lllll}-4 & -5 & 18 & 391 & 415\end{array}$

$\begin{array}{lllll}-3 & -5 & 18 & 549 & 567\end{array}$

$\begin{array}{lllll}-2 & -5 & 18 & 292 & 303\end{array}$

$\begin{array}{lllll}-1 & -5 & 18 & 337 & 328\end{array}$

$\begin{array}{lllll}0 & -5 & 18 & 774 & 771\end{array}$

$\begin{array}{lllll}1 & -5 & 18 & 287 & 336\end{array}$

$\begin{array}{lllll}2 & -5 & 18 & 998 & 975\end{array}$

$\begin{array}{lllll}3 & -5 & 18 & 308 & 292\end{array}$

$\begin{array}{lllll}4 & -5 & 18 & 600 & 624\end{array}$

$\begin{array}{lllll}-5 & -4 & 18 & 335 & 358\end{array}$ $\mathrm{h} k \mathrm{k}$ 10FO 10FC

$\begin{array}{lllll}2 & -4 & 18 & 686 & 719\end{array}$

$\begin{array}{lllll}4 & -4 & 18 & 469 & 509\end{array}$

$\begin{array}{lllll}-6 & -3 & 18 & 529 & 533\end{array}$

$\begin{array}{lllll}-5 & -3 & 18 & 683 & 693\end{array}$

$\begin{array}{lllll}-2 & -3 & 18 & 395 & 393\end{array}$

$\begin{array}{lllll}-1 & -3 & 18 & 247 & 209\end{array}$

$\begin{array}{lllll}0 & -3 & 18 & 761 & 762\end{array}$

$\begin{array}{lllll}4 & -3 & 18 & 262 & 270\end{array}$

$\begin{array}{lllll}-6 & -2 & 18 & 772 & 781\end{array}$

$\begin{array}{lllll}-5 & -2 & 18 & 728 & 688\end{array}$

$\begin{array}{lllll}-4 & -2 & 18 & 728 & 750\end{array}$

$\begin{array}{lllll}-3 & -2 & 18 & 363 & 369\end{array}$

$\begin{array}{lllll}-2 & -2 & 18 & 501 & 529\end{array}$

$\begin{array}{lllll}-1 & -2 & 18 & 356 & 329\end{array}$

$\begin{array}{lllll}0 & -2 & 18 & 878 & 859\end{array}$

$\begin{array}{lllll}1 & -2 & 18 & 405 & 375\end{array}$

$\begin{array}{lllll}2 & -2 & 18 & 880 & 891\end{array}$

$\begin{array}{lllll}-6 & -1 & 18 & 640 & 634\end{array}$

$\begin{array}{lllll}-5 & -1 & 18 & 233 & 233\end{array}$

$\begin{array}{lllll}-4 & -1 & 18 & 1038 & 1055\end{array}$

$\begin{array}{lllll}-3 & -1 & 18 & 391 & 381\end{array}$

$\begin{array}{lllll}-2 & -1 & 18 & 637 & 622\end{array}$

$\begin{array}{llllll}-1 & -1 & 18 & 401 & 405\end{array}$

$\begin{array}{llll}-1 & 18 & 685 & 669\end{array}$

$\begin{array}{lllll}1 & -1 & 18 & 969 & 930\end{array}$

$\begin{array}{lllll}2 & -1 & 18 & 1123 & 1145\end{array}$

$\begin{array}{lllll}3 & -1 & 18 & 556 & 545\end{array}$

$\begin{array}{lllll}4 & -1 & 18 & 267 & 236\end{array}$

$\begin{array}{lllll}5 & -1 & 18 & 419 & 427\end{array}$

$\begin{array}{lllll}7 & -1 & 18 & 369 & 314\end{array}$

$\begin{array}{lllll}-4 & 0 & 18 & 649 & 651\end{array}$

$\begin{array}{lllll}-2 & 0 & 18 & 731 & 729\end{array}$

$\begin{array}{lllll}-1 & 0 & 18 & 308 & 333\end{array}$

$\begin{array}{lllll}1 & 0 & 18 & 424 & 433\end{array}$ h k 1 10FO 10F'C

$\begin{array}{lllll}1 & 0 & 18 & 1040 & 1037\end{array}$

$\begin{array}{llll}0 & 18 & 499 & 515\end{array}$

$\begin{array}{lllll}3 & 0 & 18 & 1032 & 1052\end{array}$

$\begin{array}{lllll}5 & 0 & 18 & 741 & 769\end{array}$

$\begin{array}{llll}0 & 18 & 319 & 315\end{array}$

$\begin{array}{lllll}7 & 0 & 18 & 705 & 686\end{array}$

$\begin{array}{lllll}-6 & 1 & 18 & 338 & 307\end{array}$

$\begin{array}{lllll}-5 & 1 & 18 & 274 & 228\end{array}$

$\begin{array}{lllll}-3 & 1 & 18 & 423 & 448\end{array}$

$\begin{array}{lllll}-2 & 1 & 18 & 595 & 580\end{array}$

$\begin{array}{lllll}-1 & 1 & 18 & 293 & 268\end{array}$

$1118 \quad 906 \quad 893$

$\begin{array}{lllll}2 & 1 & 18 & 222 & 142\end{array}$

$\begin{array}{lllll}3 & 1 & 18 & 1003 & 1006\end{array}$

$\begin{array}{lllll}5 & 1 & 18 & 680 & 693\end{array}$

$\begin{array}{lllll}6 & 1 & 18 & 593 & 593\end{array}$

$\begin{array}{lllll}7 & 1 & 18 & 816 & 770\end{array}$

$\begin{array}{lllll}8 & 1 & 18 & 739 & 729\end{array}$

$\begin{array}{lllll}-4 & 2 & 18 & 461 & 471\end{array}$

$\begin{array}{lllll}-3 & 2 & 18 & 218 & 123\end{array}$

$\begin{array}{lllll}1 & 2 & 18 & 374 & 379\end{array}$

$\begin{array}{lllll}3 & 2 & 18 & 698 & 711\end{array}$

$\begin{array}{lllll}5 & 2 & 18 & 426 & 415\end{array}$

$\begin{array}{lllll}6 & 2 & 18 & 342 & 337\end{array}$

$\begin{array}{lllll}7 & 2 & 18 & 293 & 317\end{array}$

$\begin{array}{lllll}8 & 2 & 18 & 811 & 776\end{array}$

$\begin{array}{lllll}-5 & 3 & 18 & 531 & 554\end{array}$

$\begin{array}{lllll}-4 & 3 & 18 & 588 & 605\end{array}$

$\begin{array}{lllll}-3 & 3 & 18 & 336 & 362\end{array}$

$\begin{array}{lllll}-1 & 3 & 18 & 476 & 459\end{array}$

$\begin{array}{lllll}0 & 3 & 18 & 265 & 257\end{array}$

$\begin{array}{lllll}1 & 3 & 18 & 536 & 516\end{array}$

$\begin{array}{lllll}2 & 3 & 18 & 244 & 164\end{array}$

$\begin{array}{lllll}8 & 3 & 18 & 597 & 596\end{array}$ 
418381410

$\begin{array}{lllll}9 & 4 & 18 & 303 & 248\end{array}$

$\begin{array}{lllll}-2 & 5 & 18 & 258 & 271\end{array}$

$\begin{array}{lllll}-1 & 5 & 18 & 471 & 449\end{array}$

$\begin{array}{lllll}0 & 5 & 18 & 604 & 606\end{array}$

$\begin{array}{lllll}1 & 5 & 18 & 689 & 663\end{array}$

$\begin{array}{lllll}2 & 5 & 18 & 924 & 906\end{array}$

$\begin{array}{lllll}3 & 5 & 18 & 830 & 814\end{array}$

$\begin{array}{lllll}4 & 5 & 18 & 416 & 406\end{array}$

$\begin{array}{lllll}6 & 5 & 18 & 588 & 592\end{array}$

$\begin{array}{lllll}8 & 5 & 18 & 337 & 321\end{array}$

$\begin{array}{lllll}-3 & 6 & 18 & 624 & 633\end{array}$

$\begin{array}{lllll}-1 & 6 & 18 & 405 & 395\end{array}$

o $6 \begin{array}{llll}18 & 470 \quad 403\end{array}$

2. $6 \begin{array}{llll}6 & 18 & 1091 & 1132\end{array}$

$\begin{array}{lllll}3 & 6 & 18 & 447 & 437\end{array}$

$\begin{array}{lllll}4 & 6 & 18 & 826 & 860\end{array}$

$\begin{array}{lllll}6 & 6 & 18 & 633 & 641\end{array}$

$\begin{array}{lllll}7 & 6 & 18 & 305 & 309\end{array}$

$\begin{array}{lllll}8 & 6 & 18 & 447 & 476\end{array}$

$\begin{array}{lllll}9 & 6 & 18 & 360 & 332\end{array}$

$\begin{array}{llllll}-1 & 7 & 18 & 304 & 284\end{array}$

$2718 \quad 902 \quad 844$

$\begin{array}{lllll}4 & 7 & 18 & 882 & 901\end{array}$

6 718655690 $\begin{array}{llll}8 & 18 & 768 & 717\end{array}$

$\begin{array}{lllll}0 & 9 & 18 & 287 & 292\end{array}$

$\begin{array}{lllll}0 & 10 & 18 & 435 & 403\end{array}$

$\begin{array}{lllll}1 & 10 & 18 & 306 & 267\end{array}$

$\begin{array}{lllll}2 & 10 & 18 & 481 & 482\end{array}$

$\begin{array}{lllll}3 & 10 & 18 & 285 & 277\end{array}$

$\begin{array}{lllll}5 & 10 & 18 & 237 & 244\end{array}$

$\begin{array}{lllll}7 & 10 & 18 & 355 & 349\end{array}$

$\begin{array}{lllll}8 & 10 & 18 & 236 & 201\end{array}$

$\begin{array}{lllll}1 & 11 & 18 & 553 & 543\end{array}$

$\begin{array}{lllll}2 & 11 & 18 & 511 & 482\end{array}$

$\begin{array}{lllll}3 & 11 & 18 & 607 & 583\end{array}$

$\begin{array}{lllll}4 & 11 & 18 & 455 & 457\end{array}$

$\begin{array}{lllll}5 & 11 & 18 & 395 & 364\end{array}$

$\begin{array}{lllll}7 & 11 & 18 & 523 & 511\end{array}$

$\begin{array}{lllll}3 & 12 & 18 & 699 & 684\end{array}$

$\begin{array}{lllll}5 & 12 & 18 & 558 & 544\end{array}$

$\begin{array}{lllll}-2 & -6 & 19 & 672 & 682\end{array}$

$\begin{array}{lllll}-1 & -6 & 19 & 256 & 290\end{array}$

$\begin{array}{lllll}0 & -6 & 19 & 341 & 308\end{array}$

$\begin{array}{lllll}1 & -6 & 19 & 787 & 761\end{array}$

$\begin{array}{lllll}-3 & -5 & 19 & 468 & 474\end{array}$

$\begin{array}{lllll}-2 & -5 & 19 & 704 & 670\end{array}$

$\begin{array}{lllll}-1 & -5 & 19 & 520 & 474\end{array}$

$\begin{array}{llllll}0 & -5 & 19 & 342 & 351\end{array}$
4119085096

$\begin{array}{lllll}5 & 1 & 19 & 224 & 237\end{array}$

$\begin{array}{lllll}6 & 1 & 19 & 584 & 589\end{array}$

$\begin{array}{lllll}7 & 1 & 19 & 589 & 598\end{array}$

$\begin{array}{lllll}-5 & 2 & 19 & 415 & 411\end{array}$

$\begin{array}{lllll}-2 & 2 & 19 & 480 & 489\end{array}$

$\begin{array}{lllll}-1 & 2 & 19 & 420 & 402\end{array}$

o $219409 \quad 378$

$\begin{array}{lllll}2 & 2 & 19 & 752 & 769\end{array}$

$\begin{array}{lllll}3 & 2 & 19 & 298 & 322\end{array}$

$\begin{array}{lllll}4 & 2 & 19 & 905 & 901\end{array}$

$\begin{array}{lllll}5 & 2 & 19 & 328 & 306\end{array}$

$\begin{array}{lllll}6 & 2 & 19 & 449 & 439\end{array}$

$\begin{array}{lllll}7 & 2 & 19 & 758 & 747\end{array}$

$8 \quad 2 \quad 19 \quad 400 \quad 370$

$\begin{array}{lllll}-4 & 3 & 19 & 361 & 358\end{array}$

$\begin{array}{lllll}-3 & 3 & 19 & 380 & 405\end{array}$

$\begin{array}{lllll}-1 & 3 & 19 & 222 & 236\end{array}$

$\begin{array}{lllll}2 & 3 & 19 & 237 & 189\end{array}$

$\begin{array}{lllll}3 & 3 & 19 & 260 & 302\end{array}$

$\begin{array}{lllll}4 & 3 & 19 & 451 & 423\end{array}$

$\begin{array}{lllll}6 & 3 & 19 & 291 & 284\end{array}$

$\begin{array}{lllll}7 & 3 & 19 & 429 & 419\end{array}$

$\begin{array}{lllll}-4 & 4 & 19 & 676 & 678\end{array}$

$\begin{array}{lllll}-3 & 4 & 19 & 253 & 263\end{array}$ $\begin{array}{lllll}3 & 5 & 19 & 642 & 628\end{array}$

$4 \quad 519 \quad 506 \quad 515$

$\begin{array}{llll}5 & 19 & 259 & 272\end{array}$

$\begin{array}{lllll}7 & 5 & 19 & 258 & 267\end{array}$

$\begin{array}{lllll}-2 & 6 & 19 & 703 & 709\end{array}$

$\begin{array}{lllll}0 & 6 & 19 & 286 & 255\end{array}$

$\begin{array}{llll}6 & 19 & 766 & 730\end{array}$

$\begin{array}{lllll}2 & 6 & 19 & 267 & 303\end{array}$

$\begin{array}{lllll}3 & 6 & 19 & 1019 & 1008\end{array}$

$\begin{array}{lllll}4 & 6 & 19 & 383 & 416\end{array}$

$\begin{array}{lllll}5 & 6 & 19 & 448 & 433\end{array}$

$\begin{array}{lllll}7 & 6 & 19 & 287 & 317\end{array}$

$\begin{array}{lllll}8 & 6 & 19 & 269 & 244\end{array}$

$\begin{array}{lllll}-2 & 7 & 19 & 387 & 397\end{array}$

$\begin{array}{lllll}-1 & 7 & 19 & 218 & 209\end{array}$

$\begin{array}{lllll}0 & 7 & 19 & 250 & 180\end{array}$

$\begin{array}{lllll}1 & 7 & 19 & 446 & 437\end{array}$

$\begin{array}{lllll}3 & 7 & 19 & 896 & 907\end{array}$

$\begin{array}{lllll}5 & 7 & 19 & 787 & 812\end{array}$

$\begin{array}{lllll}6 & 7 & 19 & 263 & 255\end{array}$

$\begin{array}{lllll}7 & 7 & 19 & 283 & 336\end{array}$

$\begin{array}{lllll}8 & 7 & 19 & 608 & 613\end{array}$

$\begin{array}{lllll}-1 & 8 & 19 & 309 & 277\end{array}$

$\begin{array}{lllll}2 & 8 & 19 & 275 & 193\end{array}$

$\begin{array}{llll}0 & 19 & 1204 & 1166\end{array}$

$\begin{array}{lllll}3 & 0 & 19 & 443 & 452\end{array}$

$\begin{array}{lllll}3 & 8 & 19 & 468 & 448\end{array}$

Observed ard calculated structure factors: $\quad\left[O S(C O){ }_{2} \mathrm{PPH}_{3}\right]_{2}$ (MU-I) (MN-PPH$)$

Page 40

$\begin{array}{rrrrrrrrrr}\mathrm{h} & \mathrm{k} & 1 & 10 \mathrm{FO} & 1 \text { OFC } & \mathrm{h} & \mathrm{k} & 1 & 1 \text { OFO } & 1 \text { OFC } \\ 4 & 8 & 19 & 343 & 302 & -1 & 0 & 20 & 273 & 272 \\ 5 & 8 & 19 & 646 & 663 & 0 & 0 & 20 & 405 & 435 \\ 6 & 8 & 19 & 335 & 336 & 1 & 0 & 20 & 810 & 787 \\ 7 & 8 & 19 & 243 & 245 & 2 & 0 & 20 & 366 & 373 \\ 8 & 8 & 19 & 700 & 730 & 3 & 0 & 20 & 672 & 692 \\ 5 & 9 & 19 & 251 & 264 & 4 & 0 & 20 & 315 & 336 \\ 6 & 9 & 19 & 245 & 226 & -3 & 1 & 20 & 527 & 509 \\ 2 & 10 & 19 & 347 & 320 & -2 & 1 & 20 & 784 & 784 \\ 4 & 10 & 19 & 231 & 192 & -1 & 1 & 20 & 422 & 410 \\ 2 & 11 & 19 & 515 & 489 & 0 & 1 & 20 & 477 & 465 \\ 3 & 11 & 19 & 372 & 373 & 1 & 1 & 20 & 672 & 647 \\ 4 & 11 & 19 & 381 & 353 & 2 & 1 & 20 & 279 & 244 \\ -2 & -4 & 20 & 658 & 666 & 3 & 1 & 20 & 1066 & 1033 \\ -1 & -4 & 20 & 401 & 408 & 5 & 1 & 20 & 666 & 658 \\ 0 & -4 & 20 & 554 & 538 & 6 & 1 & 20 & 226 & 280 \\ 2 & -4 & 20 & 734 & 732 & -4 & 2 & 20 & 303 & 270 \\ -2 & -3 & 20 & 313 & 324 & -3 & 2 & 20 & 512 & 539 \\ -1 & -3 & 20 & 304 & 274 & -1 & 2 & 20 & 688 & 658 \\ 0 & -3 & 20 & 348 & 304 & 0 & 2 & 20 & 379 & 381 \\ 2 & -3 & 20 & 386 & 370 & 1 & 2 & 20 & 444 & 465 \\ 3 & -3 & 20 & 343 & 357 & 2 & 2 & 20 & 266 & 257 \\ 4 & -3 & 20 & 529 & 545 & 3 & 2 & 20 & 814 & 809 \\ -4 & -2 & 20 & 557 & 543 & 4 & 2 & 20 & 427 & 484 \\ -2 & -2 & 20 & 219 & 211 & 5 & 2 & 20 & 810 & 826 \\ 2 & -2 & 20 & 210 & 173 & 6 & 2 & 20 & 485 & 524 \\ 4 & -2 & 20 & 226 & 165 & 7 & 2 & 20 & 288 & 285 \\ -4 & -1 & 20 & 889 & 870 & -2 & 3 & 20 & 251 & 247 \\ -2 & -1 & 20 & 763 & 758 & -1 & 3 & 20 & 526 & 509 \\ 0 & -1 & 20 & 317 & 340 & 1 & 3 & 20 & 406 & 399 \\ 1 & -1 & 20 & 551 & 521 & 3 & 3 & 20 & 343 & 357 \\ 2 & -1 & 20 & 530 & 527 & 4 & 3 & 20 & 494 & 533 \\ 5 & -1 & 20 & 220 & 225 & 5 & 3 & 20 & 539 & 567 \\ -4 & 0 & 20 & 783 & 821 & 6 & 3 & 20 & 479 & 464 \\ -2 & 0 & 20 & 952 & 939 & -3 & 4 & 20 & 496 & 495 \\ & & & & & & & & & \end{array}$

$\mathrm{h} k \mathrm{k}$ 10FO 10FC

$2 \quad 4 \quad 20 \quad 272 \quad 261$

$5420 \quad 243 \quad 249$

$6420 \quad 288 \quad 313$

$\begin{array}{lllll}-3 & 5 & 20 & 724 & 670\end{array}$

$\begin{array}{lllll}-1 & 5 & 20 & 556 & 558\end{array}$

$\begin{array}{lllll}0 & 5 & 20 & 342 & 337\end{array}$

$\begin{array}{lllll}1 & 5 & 20 & 339 & 268\end{array}$

$\begin{array}{lllll}2 & 5 & 20 & 658 & 649\end{array}$

$\begin{array}{lllll}3 & 5 & 20 & 550 & 559\end{array}$

$\begin{array}{lllll}-2 & 6 & 20 & 365 & 362\end{array}$

$\begin{array}{lllll}-1 & 6 & 20 & 634 & 646\end{array}$

$\begin{array}{lllll}0 & 6 & 20 & 454 & 452\end{array}$

$\begin{array}{lllll}2 & 6 & 20 & 984 & 933\end{array}$

$\begin{array}{lllll}3 & 6 & 20 & 328 & 353\end{array}$

$\begin{array}{lllll}4 & 6 & 20 & 601 & 595\end{array}$

$\begin{array}{lllll}5 & 6 & 20 & 241 & 289\end{array}$

$\begin{array}{lllll}-1 & 7 & 20 & 413 & 436\end{array}$

$\begin{array}{llll}7 & 20 & 337 & 333\end{array}$

$\begin{array}{lllll}2 & 7 & 20 & 752 & 739\end{array}$

$\begin{array}{lllll}4 & 7 & 20 & 916 & 934\end{array}$

$\begin{array}{lllll}6 & 7 & 20 & 428 & 439\end{array}$

$\begin{array}{lllll}7 & 7 & 20 & 258 & 272\end{array}$

$\begin{array}{lllll}0 & 8 & 20 & 313 & 272\end{array}$

$\begin{array}{lllll}2 & 8 & 20 & 317 & 320\end{array}$

$\begin{array}{lllll}3 & 8 & 20 & 339 & 392\end{array}$

$\begin{array}{lllll}4 & 8 & 20 & 698 & 693\end{array}$

$\begin{array}{lllll}5 & 8 & 20 & 477 & 444\end{array}$

$\begin{array}{lllll}6 & 20 & 490 \quad 533\end{array}$

$\begin{array}{lllll}7 & 8 & 20 & 436 & 427\end{array}$

$\begin{array}{lllll}1 & 9 & 20 & 241 & 206\end{array}$

$\begin{array}{lllll}3 & 9 & 20 & 252 & 214\end{array}$

$\begin{array}{lllll}4 & 9 & 20 & 278 & 294\end{array}$

$\begin{array}{lllll}5 & 9 & 20 & 524 & 504\end{array}$

$\begin{array}{lllll}6 & 9 & 20 & 334 & 306\end{array}$ h $\mathrm{k}$ I 10Fo 10Fc

$\begin{array}{lllll}-1 & -3 & 21 & 673 & 678\end{array}$

$\begin{array}{lllll}1 & -3 & 21 & 476 & 456\end{array}$

$\begin{array}{lllll}-1 & -1 & 21 & 369 & 374\end{array}$

$\begin{array}{lllll}0 & -1 & 21 & 222 & 183\end{array}$

$\begin{array}{lllll}2 & -1 & 21 & 233 & 249\end{array}$

$\begin{array}{lllll}4 & -1 & 21 & 224 & 212\end{array}$

$\begin{array}{lllll}-3 & 0 & 21 & 745 & 720\end{array}$

$\begin{array}{lllll}-2 & 0 & 21 & 419 & 443\end{array}$

$\begin{array}{lllll}-1 & 0 & 21 & 671 & 605\end{array}$

- $021 \quad 469 \quad 410$

$\begin{array}{lllll}2 & 0 & 21 & 578 & 612\end{array}$

$\begin{array}{lllll}3 & 0 & 21 & 360 & 401\end{array}$

$\begin{array}{llll}4 \quad 021 & 272 & 255\end{array}$

$\begin{array}{lllll}-3 & 1 & 21 & 563 & 533\end{array}$

$\begin{array}{lllll}-2 & 1 & 21 & 592 & 566\end{array}$

$\begin{array}{lllll}-1 & 1 & 21 & 655 & 629\end{array}$

- $12121521 \quad 486$

$\begin{array}{lllll}2 & 1 & 21 & 794 & 771\end{array}$

$\begin{array}{lllll}4 & 1 & 21 & 793 & 824\end{array}$

$\begin{array}{lllll}-2 & 2 & 21 & 744 & 718\end{array}$

$\begin{array}{lllll}-1 & 2 & 21 & 346 & 340\end{array}$

$\begin{array}{lllll}0 & 2 & 21 & 634 & 608\end{array}$

$\begin{array}{lllll}2 & 2 & 21 & 428 & 456\end{array}$

$\begin{array}{lllll}3 & 2 & 21 & 503 & 492\end{array}$

$\begin{array}{lllll}4 & 2 & 21 & 776 & 764\end{array}$

$5 \quad 2 \quad 21 \quad 438 \quad 476$

$\begin{array}{lllll}5 & 2 & 21 & 443 & 488\end{array}$

$\begin{array}{lllll}-2 & 3 & 21 & 416 & 440\end{array}$

$\begin{array}{lllll}0 & 3 & 21 & 655 & 673\end{array}$

$\begin{array}{lllll}2 & 3 & 21 & 344 & 356\end{array}$

$\begin{array}{lllll}3 & 3 & 21 & 482 & 499\end{array}$

$\begin{array}{lllll}4 & 3 & 21 & 376 & 384\end{array}$

$\begin{array}{lllll}5 & 3 & 21 & 746 & 743\end{array}$

$\begin{array}{lllll}6 & 3 & 21 & 421 & 420\end{array}$ h $\mathrm{k}$ 1OFO 1OFC

$\begin{array}{lllll}-1 & 4 & 21 & 254 & 193\end{array}$

$\begin{array}{lllll}0 & 4 & 21 & 320 & 351\end{array}$

$\begin{array}{lllll}5 & 4 & 21 & 512 & 510\end{array}$

$\begin{array}{llllll}-2 & 5 & 21 & 599 & 580\end{array}$

$\begin{array}{lllll}3 & 5 & 21 & 333 & 304\end{array}$

$\begin{array}{lllll}-1 & 6 & 21 & 425 & 443\end{array}$

$0 \quad 621500 \quad 486$

$\begin{array}{lllll}1 & 6 & 21 & 531 & 506\end{array}$

$\begin{array}{lllll}3 & 6 & 21 & 599 & 591\end{array}$

$\begin{array}{lllll}4 & 6 & 21 & 226 & 184\end{array}$

$\begin{array}{lllll}5 & 6 & 21 & 274 & 252\end{array}$

$\begin{array}{lllll}0 & 7 & 21 & 450 & 419\end{array}$

$\begin{array}{lllll}1 & 7 & 21 & 503 & 504\end{array}$

$\begin{array}{lllll}3 & 7 & 21 & 736 & 691\end{array}$

$\begin{array}{lllll}5 & 7 & 21 & 512 & 553\end{array}$

$\begin{array}{lllll}1 & 8 & 21 & 404 & 403\end{array}$

$\begin{array}{lllll}3 & 8 & 21 & 439 & 418\end{array}$

$\begin{array}{lllll}4 & 8 & 21 & 436 & 414\end{array}$

$\begin{array}{lllll}5 & 8 & 21 & 585 & 619\end{array}$

$\begin{array}{lllll}4 & 9 & 21 & 618 & 607\end{array}$

$\begin{array}{lllll}0 & -1 & 22 & 225 & 18\end{array}$

$\begin{array}{lllll}-1 & 0 & 22 & 369 & 367\end{array}$

$\begin{array}{lllll}0 & 0 & 22 & 446 & 391\end{array}$

$\begin{array}{lllll}1 & 0 & 22 & 245 & 169\end{array}$

$\begin{array}{lllll}3 & 0 & 22 & 319 & 252\end{array}$

$\begin{array}{lllll}-1 & 1 & 22 & 725 & 677\end{array}$

$\begin{array}{llll}1 & 22 & 408 & 431\end{array}$

$1 \quad 122 \quad 504 \quad 512$

$\begin{array}{lllll}3 & 1 & 22 & 645 & 624\end{array}$

$\begin{array}{lllll}-1 & 2 & 22 & 849 & 783\end{array}$

$\begin{array}{lllll}0 & 2 & 22 & 341 & 301\end{array}$

$\begin{array}{lllll}1 & 2 & 22 & 502 & 466\end{array}$

$\begin{array}{lllll}3 & 2 & 22 & 471 & 453\end{array}$

$4 \quad 222 \quad 523 \quad 523$ 
